\title{
IMPERFECT BIFURCATION FOR THE QUASI-GEOSTROPHIC SHALLOW-WATER EQUATIONS
}

\author{
DAVID GERARD DRITSCHEL, TAOUFIK HMIDI, AND CORALIE RENAULT
}

\begin{abstract}
We study analytical and numerical aspects of the bifurcation diagram of simplyconnected rotating vortex patch equilibria for the quasi-geostrophic shallow-water (QGSW) equations. The QGSW equations are a generalisation of the Euler equations and contain an additional parameter, the Rossby deformation length $\varepsilon^{-1}$, which enters in the relation between stream function and (potential) vorticity. The Euler equations are recovered in the limit $\varepsilon \rightarrow 0$. We prove, close to circular (Rankine) vortices, the persistence of the bifurcation diagram for arbitrary Rossby deformation length. However we show that the two-fold branch, corresponding to Kirchhoff ellipses for the Euler equations, is never connected even for small values $\varepsilon$, and indeed is split into a countable set of disjoint connected branches. Accurate numerical calculations of the global structure of the bifurcation diagram and of the limiting equilibrium states are also presented to complement the mathematical analysis.
\end{abstract}

\section{Contents}

1. Introduction 2

2. Numerical approach 3

3. Numerical results $\quad 7$

$\begin{array}{ll}3.1 . & \text { 2-fold vortex patch equilibria }\end{array}$

3.2. 3 -fold vortex patch equilibria $\quad 9$

4. Tools used for the mathematical analysis 14

4.1. Notation 14

4.2. Modified Bessel functions 14

4.3. Boundary equations 16

5. Bifurcation to $m$-fold symmetric vortex patch equilibria $\quad 17$

5.1. Main result 17

5.2. Crandall-Rabinowitz's Theorem with a parameter 18

5.3. Function spaces I 21

5.4. Regularity of the functional I 21

5.5. Spectral study 22

6. Imperfect bifurcation close to the branch of Kirchhoff ellipses 27

6.1. Function spaces II 28

6.2. Summary of the bifurcations from Kirchhoff ellipses 28

6.3. Regularity of the functional II 29

6.4. Bifurcation diagram far from the resonant set 31

6.5. Breakdown of the bifurcation diagram close to the resonant set 35

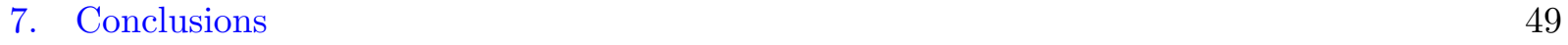

$\begin{array}{ll}\text { References } & 50\end{array}$ 


\section{INTRODUCTION}

In this paper we investigate new aspects of simply-connected vortex patch relative equilibria satisfying the quasi-geostrophic shallow water (QGSW) equations. These equations are derived asymptotically from the rotating shallow water equations, in the limit of rapid rotation and weak variations of the free surface [28]. A key property of these equations, and of the parent shallow water equations, is the material conservation of 'potential vorticity', $q$, a quantity which remains unchanged following fluid particles. The QGSW equations are given by

$$
\left\{\begin{array}{l}
\partial_{t} q+v \cdot \nabla q=0,(t, x) \in \mathbb{R}_{+} \times \mathbb{R}^{2}, \\
v=\nabla^{\perp} \psi \\
\psi=\left(\Delta-\varepsilon^{2}\right)^{-1} q, \\
q_{\mid t=0}=q_{0}
\end{array}\right.
$$

where $v$ refers to the velocity field, $\psi$ is the stream function, $\nabla^{\perp}=\left(-\partial_{2}, \partial_{1}\right)$ and $\varepsilon \in \mathbb{R}$. The parameter $\varepsilon$, when it is positive, is known as the inverse 'Rossby deformation length', a natural length scale arising from a balance between rotation and stratification. Small $\varepsilon$ physically corresponds to a free surface which is nearly rigid. For $\varepsilon=0$, we recover the two-dimensional Euler equations.

Historically, substantially more is known about vortex patch relative equilibria for the Euler equations than for the QGSW equations. The most famous example is the exact analytical solution for a rotating ellipse, found by Kirchhoff in 1876 [17]. This is in fact a non-trivial family of solutions bifurcating from Rankine's vortex, the circular patch (all axisymmetric vorticity distributions are in equilibrium, by symmetry). Kirchhoff's elliptical vortex is linearly stable when its aspect ratio $\lambda \in[1 / 3,1]$, i.e. when it is not strongly deformed from a circle, as was shown in 1893 by Love [19]. Love also discovered that there is a sequence of instabilities, at $\lambda=\lambda_{m}$ for $m=3,4, \ldots$, ordered by the elliptical coordinate azimuthal wavenumber $m$. That is, $\lambda_{3}>\lambda_{4}>\ldots$, with $\lambda_{m} \rightarrow 0$ as $m \rightarrow \infty$ [7]. Later, it was discovered that new branches of vortex patch equilibria bifurcate from each of these instability points [4,20], revealing that the equilibrium solutions of Euler's equations are exceedingly rich and varied. Analytical proofs and results on the boundary regularity were given in $[2,3,14,15]$.

The ellipse is not the only solution which bifurcates from a circle. Deem and Zabusky in 1978 [6] discovered, by numerical methods, $m$-fold symmetric vortex patch solutions for $m>2$ which are the generalizations of Kirchhoff's ellipse. The near-circular solutions resemble small-amplitude boundary waves. Their existence was proved analytically by Burbea [1] using a conformal mapping technique and bifurcation theory. At larger wave amplitudes, the outward protruding crests of the waves sharpen, ultimately limiting in a shape with right-angle corners. These corners coincide with hyperbolic stagnation points in the rotating reference frame in which the patch is steady. From an analytical point of view, this problem is still open and some progress has been recently made in [13].

In the present study, we generalize these vortex patch solutions further by exploring how they are altered for the QGSW equations when $\varepsilon \in \mathbb{R}^{*}$ (the Euler case corresponds to $\varepsilon=0$ ). We focus on $\varepsilon \ll 1$ where analytical progress can be made, but also present numerical solutions that confirm the analysis and extend it to larger positive $\varepsilon$. A surprising discovery is a new branch of $m$-fold symmetric solutions which do not bifurcate from the circle (below we exhibit the case $m=3$ ). This is an isolated branch and exists even for $\varepsilon=0$.

Some relevant vortex patch solutions for the QGSW equations are already available in the literature. Polvani (1988) [24] and Polvani, Zabusky and Flierl (1989) [25] computed the generalization 
of Kirchhoff's ellipse for various values of $\varepsilon$ (as well as for doubly-connected patches and for multi-layer flows). Later, Płotka and Dritschel (2012) [23] carried out a more comprehensive analysis of the generalized Kirchhoff ellipse solutions, including linear stability and nonlinear evolution. In these studies, solutions were obtained numerically, starting near the known circular patch solution. In all cases, the limiting states were found to be dumbbell-shaped, specifically two symmetrical teardrop-shaped patches connected at a single point. This is in stark contrast with Kirchhoff's ellipse, which continues as a solution at arbitrarily small aspect ratio $\lambda$.

In fact, as shown in this study, there are other two-fold symmetric vortex patch solutions of the QGSW equations. However, these are not on the branch of solutions connected to the circular patch. We demonstrate numerically, then prove mathematically, that the Kirchhoff branch for $\varepsilon=0$ splits up into many disconnected branches for any $\varepsilon>0$. The limiting dumbbell state found in the above studies is directly related to the limiting state of just one of the solution branches bifurcating from the Kirchhoff ellipse at $\lambda=\lambda_{4}$ found by Luzzatto-Fegiz and Williamson (2010) [20] for $\varepsilon=0$. The other branch lies on a disconnected branch of solutions when $\varepsilon>0$. The same behavior appears to occur near all other even bifurcations, i.e. near $\lambda=\lambda_{6}, \lambda_{8}, \ldots$, though we only have numerical evidence for this at present.

The plan of the paper is as follows. In section 2, we describe the numerical method employed to compute the vortex patch solutions. Results are then presented in section 3, both for the two-fold and the three-fold singly-connected vortex patch solutions. The remainder of the paper is devoted to proving the existence of $m$-fold solutions (in section 5 ), and proving that the two-fold solution branch splits near $\lambda=\lambda_{4}$ when $|\varepsilon| \ll 1$ (in section 6). We conclude in section 7 and suggest ideas for future work.

\section{NumericAl APPROACH}

To navigate along equilibrium solution branches up to limiting states having corners on the patch boundaries, it is necessary to employ a robustly convergent numerical method. Here we follow Luzzatto-Fegiz and Williamson (2011) [21], employing a Newton iteration to find corrections to the boundary shape from the exact condition

$$
\psi(\boldsymbol{x})-\frac{1}{2} \Omega|\boldsymbol{x}|^{2}=C
$$

expressing the fact that the stream functionstream function is constant in a frame of reference rotating at the angular velocity $\Omega$. Here $C$ is a constant, and in this section only $\boldsymbol{x}$ refers to the vector position of a point with coordinates $(x, y)$. For a single patch of uniform (potential) vorticity $q=2 \pi$, the stream function $\psi$ is determined from the contour integral

$$
\psi(\boldsymbol{x})=\oint_{\mathcal{C}} H(\varepsilon r)\left(\left(x^{\prime}-x\right) \mathrm{d} y^{\prime}-\left(y^{\prime}-y\right) \mathrm{d} x^{\prime}\right)
$$

where $\mathcal{C}$ refers to the boundary of the patch, $r=\left|\boldsymbol{x}^{\prime}-\boldsymbol{x}\right|$ is the distance between $\boldsymbol{x}^{\prime}$ and $\boldsymbol{x}$, and the function $H$ is given by

$$
H(z)=\frac{z K_{1}(z)-1}{z^{2}}
$$

where $K_{1}$ is the modified Bessel function of order 1 [23]. In the limit $z \rightarrow 0$, relevant to the Euler equations, $H(\varepsilon r)$ reduces to $\frac{1}{4}\left(\ln r^{2}-1\right)$ plus an unimportant constant.

We start with a guess $\boldsymbol{x}=\overline{\boldsymbol{x}}(\vartheta)$ for the shape of $\mathcal{C}$. Here, following [10], we use a special coordinate $\vartheta$ proportional to the travel time of a fluid particle around $\mathcal{C}$ from some pre-assigned starting point. 
This coordinate simplifies the equations which must be solved at each step of the iterative procedure correcting the boundary shape. The travel time $t$ is computed from

$$
t=\int_{0}^{s} \frac{\mathrm{d} s^{\prime}}{\left|\tilde{\boldsymbol{u}}\left(s^{\prime}\right)\right|}
$$

where $s$ is arc length measured from the starting point, and $\tilde{\boldsymbol{u}}=\nabla^{\perp}\left(\psi-\frac{1}{2} \Omega|\boldsymbol{x}|^{2}\right)$ is the velocity in the rotating frame of reference (here $\nabla^{\perp}=(-\partial / \partial y, \partial / \partial x)$ is the skewed gradient operator). The integral around the entire boundary, $T_{p}$, gives the particle orbital period, from which we define the particle frequency $\Omega_{p}=2 \pi / T_{p}$, a particularly useful diagnostic in which to characterize the equilibrium patch solutions. From $\Omega_{p}$, we define the travel-time coordinate as $\vartheta=\Omega_{p} t$, where $t$ is given in (2.4). Notably, at equilibrium, $\tilde{\boldsymbol{u}}$ is tangent to $\mathcal{C}$. During the iteration to find an equilibrium, this will not be exactly true, but the small discrepancy has no significant effect on the convergence of the numerical procedure.

We correct the previous guess $\overline{\boldsymbol{x}}$ at each step of the iteration by taking

$$
\boldsymbol{x}=\overline{\boldsymbol{x}}+\frac{\hat{\eta}(\vartheta)\left(\bar{y}_{\vartheta},-\bar{x}_{\vartheta}\right)}{\left|\overline{\boldsymbol{x}}_{\vartheta}\right|^{2}}
$$

where a $\vartheta$ subscript denotes differentiation with respect to $\vartheta$. This represents a normal perturbation to the previous boundary, though the scalar function $\hat{\eta}$ has units of area. This choice of perturbation leads to the simplest form for the linearized approximation to (2.1) and (2.2),

$$
\Omega_{p} \hat{\eta}(\vartheta)-\int_{0}^{2 \pi} \hat{\eta}\left(\vartheta^{\prime}\right) K_{0}\left(\varepsilon\left|\overline{\boldsymbol{x}}\left(\vartheta^{\prime}\right)-\overline{\boldsymbol{x}}(\vartheta)\right|\right) \mathrm{d} \vartheta^{\prime}-\frac{1}{2} \hat{\Omega}|\overline{\boldsymbol{x}}(\vartheta)|^{2}=C-\bar{\psi}(\vartheta)+\frac{1}{2} \bar{\Omega}|\overline{\boldsymbol{x}}(\vartheta)|^{2} \equiv R(\vartheta)
$$

where terms only up to first order in $\hat{\eta}$ have been retained, and we have additionally included a perturbation $\hat{\Omega}$ in the rotation rate $\Omega=\bar{\Omega}+\hat{\Omega}$. Above, $K_{0}$ is the modified Bessel function of order 0 , and notably $K_{0}(\varepsilon r)$ reduces to $-\ln r$ plus an unimportant constant in the limit $\varepsilon \rightarrow 0$. On the right hand side of $(2.6), \bar{\psi}$ refers to the stream function evaluated using the previous guess $\overline{\boldsymbol{x}}$, i.e. using $\overline{\boldsymbol{x}}$ in place of $\boldsymbol{x}$ in (2.2). This is a linear integral equation for $\hat{\eta}$ and possibly $\hat{\Omega}$, but its solution requires additional constraints. First of all, we require that the vortex patch area $A$ remains constant, and without loss of generality we may take $A=\pi$. This constraint gives rise to an additional equation after linearising the expression for area

$$
A=\frac{1}{2} \oint_{\mathcal{C}} x \mathrm{~d} y-y \mathrm{~d} x
$$

leading to

$$
\int_{0}^{2 \pi} \hat{\eta}(\vartheta) \mathrm{d} \vartheta=A-\bar{A}
$$

where $\bar{A}$ is the area of the previous guess found by using $\overline{\boldsymbol{x}}$ in place of $\boldsymbol{x}$ in (2.7). If $\Omega$ is held fixed during the iteration $(\hat{\Omega}=0)$, no further constraints are necessary. However, holding $\Omega$ fixed does not allow one to negotiate turning points in the equilibrium solution branches [21]. If we let $\Omega$ vary (and thus be determined as part of the solution), we need to impose a further constraint. The most natural is angular impulse:

$$
J=\frac{1}{4} \oint_{\mathcal{C}}|\boldsymbol{x}|^{2}(x \mathrm{~d} y-y \mathrm{~d} x)
$$

whose linearisation leads to the additional equation

$$
\int_{0}^{2 \pi}|\overline{\boldsymbol{x}}(\vartheta)|^{2} \hat{\eta}(\vartheta) \mathrm{d} \vartheta=J-\bar{J}
$$


where $\bar{J}$ is the angular impulse of the previous guess found by using $\overline{\boldsymbol{x}}$ in place of $\boldsymbol{x}$ in (2.9).

Numerically, the vortex patch boundary for an $m$-fold equilibrium is represented by $n=400 \mathrm{~m}$ boundary nodes, approximately equally spaced in $\vartheta$. Upon each iteration, the travel time coordinate is recomputed and the nodes are redistributed to be equally-spaced in $\vartheta$, to within numerical discretisation error. The algorithm for node redistribution is otherwise the same as that introduced in [8] and uses local cubic splines for high accuracy. Also, the calculation of the stream function $\psi$ and velocity $\boldsymbol{u}=\nabla^{\perp} \psi$ by contour integration is described in [8], and further in [9] for the QGSW equations (as used by [23] to determine the branch of 2-fold QGSW equilibria bifurcating from the Rankine vortex).

The perturbation function $\hat{\eta}(\vartheta)$ is represented as the truncated Fourier series

$$
\hat{\eta}(\vartheta)=\sum_{j=0}^{N} a_{j} \cos (j m \vartheta)
$$

which imposes even symmetry. The same symmetry is imposed for the node redistribution, so only 201 boundary nodes are unique. Not all vortex patch equilibria have such symmetry [21], but we restrict attention to symmetric equilibria in this study. A truncation of $N=32$ was found sufficient to produce results accurate to within the plotted line widths below. Accuracy is not significantly improved when using larger $N$ because ultimately the highest modes $\cos (j m \vartheta)$ for $j$ near $N$ are poorly represented by the boundary nodes. In general, we find $N \sim 0.08 n / m$ ensures the highest modes are adequately resolved, as judged by the decay of the Fourier coefficients $a_{j}$ for $j$ large.

Note the coefficient $a_{0}$ is directly determined by area conservation. From (2.8), we find

$$
a_{0}=\frac{A-\bar{A}}{2 \pi} .
$$

This means that the stream function constant $C$ is determined by averaging (2.6) over $\vartheta$, and thus $C$ is generally determined from all of the $a_{n}$ and $\hat{\Omega}$. However, $C$ is not needed to find an equilibrium, so this calculation need not be done.

We solve (2.6) for $a_{n}(n>0)$ - together with (2.10) for $\hat{\Omega}$ when $\Omega$ is allowed to vary — by substituting (2.11) into (2.6), then multiplying both sides by $\pi^{-1} \cos (i m \vartheta)$, and finally integrating over $\vartheta$. This results in the linear system

$$
\sum_{j=1}^{N} A_{i j} a_{j}-B_{i} \hat{\Omega}=C_{i}, \quad i=1,2, \ldots, N
$$

where the matrix elements $A_{i j}$ and vector components $B_{i}$ and $C_{i}$ are given by

$$
\begin{aligned}
A_{i j} & =\Omega_{p} \delta_{i j}-\frac{1}{\pi} \int_{0}^{2 \pi} \int_{0}^{2 \pi} \cos (i m \vartheta) \cos \left(j m \vartheta^{\prime}\right) K_{0}\left(\varepsilon\left|\overline{\boldsymbol{x}}\left(\vartheta^{\prime}\right)-\overline{\boldsymbol{x}}(\vartheta)\right|\right) \mathrm{d} \vartheta^{\prime} \mathrm{d} \vartheta \\
B_{i} & =\frac{1}{2 \pi} \int_{0}^{2 \pi}|\overline{\boldsymbol{x}}(\vartheta)|^{2} \cos (i m \vartheta) \mathrm{d} \vartheta \\
C_{i} & =\frac{1}{\pi} \int_{0}^{2 \pi} R(\vartheta) \cos (i m \vartheta) \mathrm{d} \vartheta
\end{aligned}
$$

where $\delta_{i j}$ is the Kronecker delta. Notably, $A_{i j}=A_{j i}$. Care is taken to avoid the logarithmic singularity in $K_{0}(\varepsilon r)$ by separating this function into a singular part $S=-\ln \left(1-\cos \left(\vartheta^{\prime}-\vartheta\right)\right)$, 
which can be integrated analytically (and contributes $-\pi \delta_{i j} /(i m)$ to $A_{i j}$ ), and a non-singular remainder $K_{0}-S$ which is integrated by two-point Gaussian quadrature. The same numerical quadrature is used to compute $B_{i}$ (if $\hat{\Omega} \neq 0$ ) and $C_{i}$. The area constant $a_{0}$ from (2.12) is ignored at this stage and is added after the linear system (2.13) is solved.

When $\Omega$ is held fixed, we have $\hat{\Omega}=0$ in (2.13) above, and then (2.13) may be directly solved for the coefficients $a_{j}, j=1,2, \ldots, N$. Otherwise, we need to add another equation in order to also obtain $\hat{\Omega}$. We do this by fixing the angular impulse and thus use (2.10). Inserting (2.11) into (2.10) and dividing by $2 \pi$ results in the further linear equation

$$
\sum_{j=1}^{N} B_{j} a_{j}=\frac{J-\bar{J}}{2 \pi}
$$

(again ignoring the area constant $a_{0}$ ). If we tag $-\hat{\Omega}$ to the end of the vector of coefficients $\left(a_{1}, a_{2}, \ldots, a_{N}\right)$, we obtain a symmetric linear system for this vector, which is easily solved by standard numerical linear algebra packages. Only at this stage do we add $a_{0}$ from (2.12) and fully determine $\hat{\eta}(\vartheta)$ from the sum in (2.11). We then obtain a new guess for the vortex boundary shape from (2.5), and accept this as the converged solution if the maximum value in $|\boldsymbol{x}-\overline{\boldsymbol{x}}|<10^{-7}$. Otherwise, we use $\boldsymbol{x}$ as the next guess $\overline{\boldsymbol{x}}$ and repeat the above procedure.

The above explains how we obtain a single equilibrium state for either fixed rotation rate $\Omega$ or fixed angular impulse $J$. To obtain a whole family of states or solutions, after convergence to one state, we slightly change either $\Omega$ or $J$ and search for the next state using the same iterative procedure described above.

This approach fails when we reach a turning point in either $\Omega$ or $J$. When this happens, we switch the control parameter (e.g. instead of changing $\Omega$ we change $J$ ) and continue to the next turning point. Fortunately, the turning points for $\Omega$ and $J$ are almost never coincident, so this is an effective strategy. A more elaborate strategy was followed in [21], but the proposed strategy has been found to be highly effective. We always reach limiting states containing near corners on their boundaries, beyond which there are no other states with the same topology.

We note for completeness that the above procedure is readily generalized to study multiplyconnected vortex patch equilibria. The primary change is that the constant $C$ and all functions of $\vartheta$ in (2.6) acquire a $k$ subscript (denoting the $k$ th patch boundary or contour), while all functions of $\vartheta^{\prime}$ acquire an $\ell$ subscript. Furthermore, the integral is now summed over all contours $\ell$ and multiplied by the uniform potential vorticity $q_{\ell}$. Each contour must also satisfy an area constraint like (2.8), with $\eta$ and $A$ supplemented by $k$ subscripts. If angular impulse is fixed, additionally (2.10) must be satisfied. Here again all functions of $\vartheta$ acquire a $k$ subscript, and the integral must be summed over $k$ and multiplied by the uniform potential vorticity $q_{k}$. The impulse is an invariant of the entire vortex system.

The procedure also generalizes to cases in which the vortex patches steadily translate rather than rotate. In this case, without loss of generality, we may suppose that the patches translate in the $x$ direction at speed $U$. The only changes then required in the procedure described above is to replace $\frac{1}{2} \Omega|\overline{\boldsymbol{x}}|^{2}$ by $-U \bar{y}$ and to impose linear impulse conservation,

$$
I=\frac{1}{3} \sum_{k} q_{k} \oint_{\mathcal{C}_{k}} y_{k}\left(x_{k} \mathrm{~d} y_{k}-y_{k} \mathrm{~d} x_{k}\right)
$$


When searching for equilibria of a fixed linear impulse $I$, an additional equation is required to determine the perturbation $\hat{U}$ to the speed $U$. This is found by linearising (2.16) after substituting (2.5), supplemented by $k$ subscripts, yielding

$$
\sum_{k} q_{k} \int_{0}^{2 \pi} \bar{y}_{k}(\vartheta) \hat{\eta}_{k}(\vartheta) \mathrm{d} \vartheta=I-\bar{I}
$$

where $\bar{I}$ is the linear impulse of the previous guess found by using $\overline{\boldsymbol{x}}_{k}$ in place of $\boldsymbol{x}_{k}$ in (2.16).

Various diagnostics may be used to characterize the equilibrium vortex patch solutions. For the symmetric $m$-fold solutions studied here, the only other non-zero invariant besides angular impulse is the 'excess' energy $E$, as defined in [23]. For a single patch of area $A=\pi$, this is determined from

$$
E=\frac{\pi}{4}(\ln (\varepsilon / 2)+\gamma)-\frac{1}{4 \pi} \oint_{\mathcal{C}} \oint_{\mathcal{C}} H(\varepsilon r)\left[\left(\boldsymbol{x}^{\prime}-\boldsymbol{x}\right) \cdot \mathrm{d} \boldsymbol{x}^{\prime}\right]\left[\left(\boldsymbol{x}^{\prime}-\boldsymbol{x}\right) \cdot \mathrm{d} \boldsymbol{x}\right]
$$

where $\gamma=0.5772 \ldots$ is Euler's constant, $r=\left|\boldsymbol{x}-\boldsymbol{x}^{\prime}\right|$, and $H(z)$ is defined in (2.3). This expression for $E$ has a finite limit as $\varepsilon \rightarrow 0$, and equals the excess energy for the Euler equations (see discussion in appendix $\mathrm{B}$ of [23]). For a circular patch, $E=\pi / 16$ when $\varepsilon=0$.

The energy and angular impulse are important since minima or maxima of these quantities, as a function of a control parameter like $\Omega$, generally indicate changes in stability. In the results below, we have confirmed this by a direct linear stability analysis outlined in [10] and used previously in $[23]$.

\section{NumericAl RESUlts}

3.1. 2-fold vortex patch equilibria. We begin by discussing 2-fold symmetric vortex patch equilibria, in particular the structure of the solution branches for small $\varepsilon$. This structure is illustrated in two ways for $\varepsilon=0.01$ in figure 1 .

This figure shows the difficulty in distinguishing solution branches when a conserved quantity like $J$ or $E$ is plotted versus the control parameter $\Omega$. On the other hand, $\Omega_{p}$ versus $\Omega$ fully opens the branching structure, enabling one to see the separation in the branch stemming from the circular vortex having $\Omega=\frac{1}{4}$ (beyond the upper right corner of the figure) from the next branch. In panel (b), the separate branches are coloured, with the primary one being black, the second one blue (partly overlaid by the third one in red), etc. The blue, red, green and cyan branches all have the same general form. They rise at larger $\Omega$ from small $\Omega_{p}$, reach a maximum in $\Omega_{p}$, then fall at smaller $\Omega$. If we could reach the limiting states having one or more stagnation points on the vortex boundary (where the boundary exhibits a corner), we would find $\Omega_{p}=0$ since it would take an infinite time for a particle to circulate around the boundary.

The separation of these branches becomes increasingly difficult to see as $\Omega$ decreases; however, at the larger value of $\varepsilon=0.1$ (shown in figure 2), we can clearly see the second branch separating from the third. We believe this is a generic feature for all $\varepsilon>0$ : all branches separate.

The uppermost branch of solutions stemming from the circular vortex was computed previously by [23], and at that time was thought to be the only branch of 2 -fold solutions. The limiting state is a dumbbell shaped vortex touching at a single point at the origin. On the second branch, there are two limiting states. The one having the largest $\Omega$ has a rugby-ball shape with right-angled corners at the outermost tips. The other limiting state has the form of an 
(a)

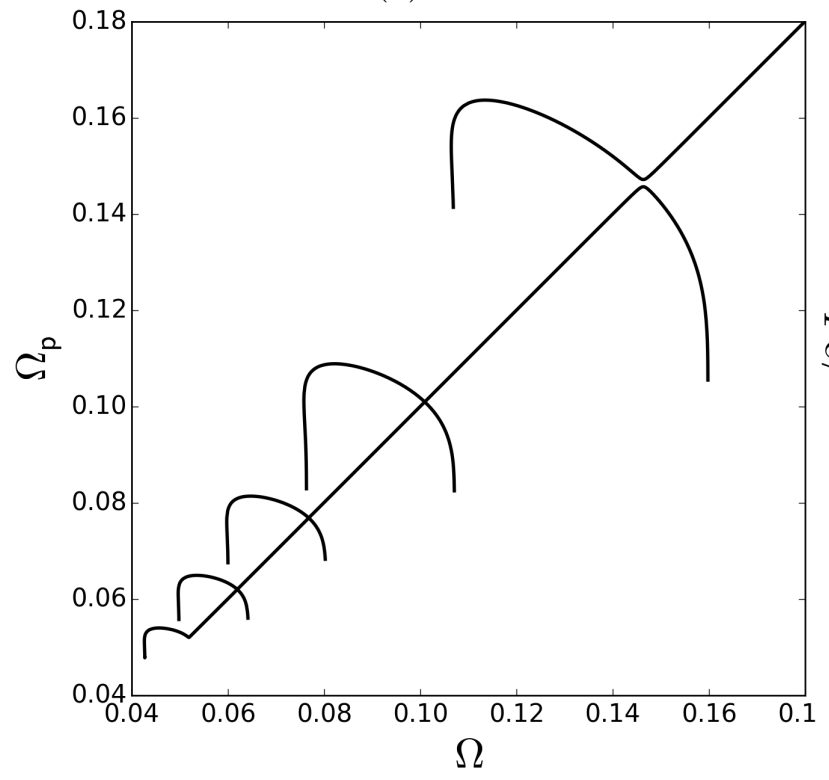

(b)

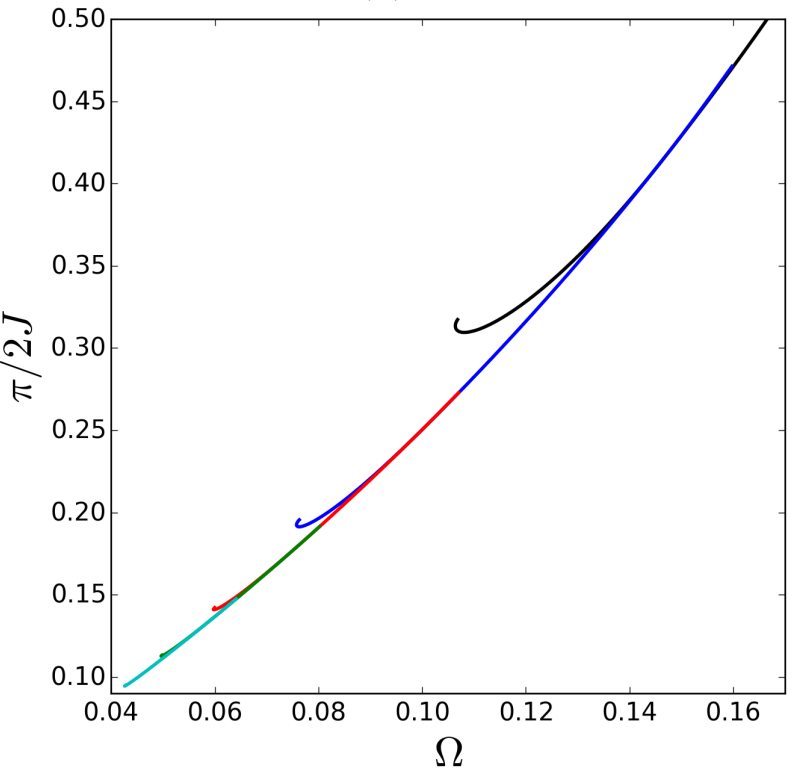

FigURE 1. Solution branch structure for 2 -fold vortex patch equilibria when $\varepsilon=$ 0.01 . In (a), we shown the particle frequency $\Omega_{p}$ versus rotation rate $\Omega$, while in (b) we show $\pi / 2 J$ versus $\Omega$ and render separate branches in different colours. Note, for a circular vortex patch, $J=\pi / 2$. The upper right branch is not shown in its entirety; it begins at the circular patch with $\Omega=\frac{1}{4}$ and $\Omega_{p}=\frac{1}{4}$. Also, only the first five branches of an infinite set of them converging on $\Omega=\Omega_{p}=0$ are shown.

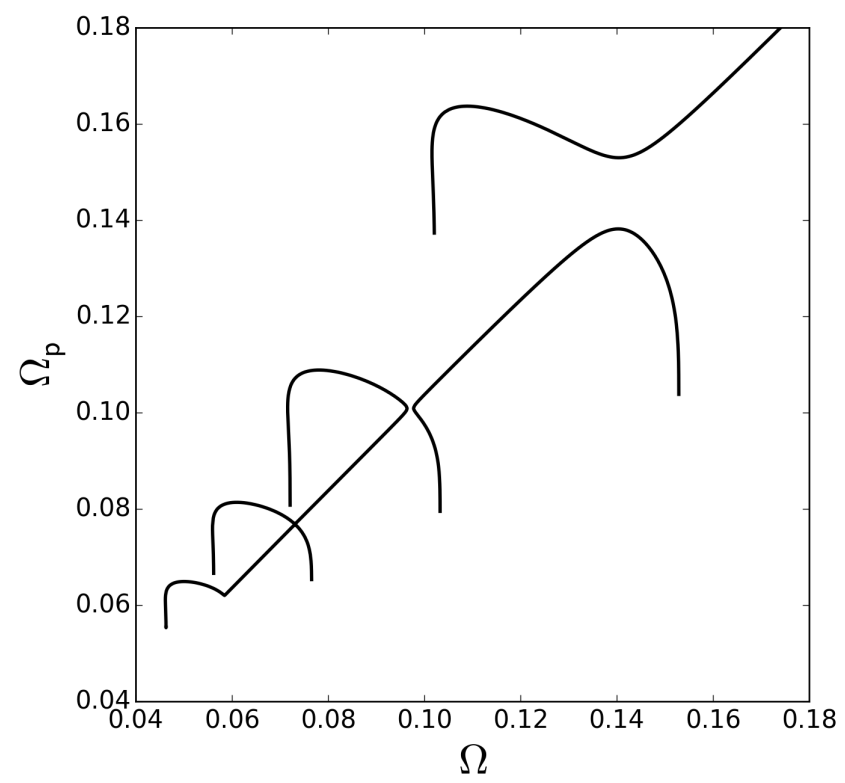

FigURE 2. Solution branch structure for 2-fold vortex patch equilibria when $\varepsilon=0.1$. Here, only $\Omega_{p}$ versus $\Omega$ is shown. 
(a)

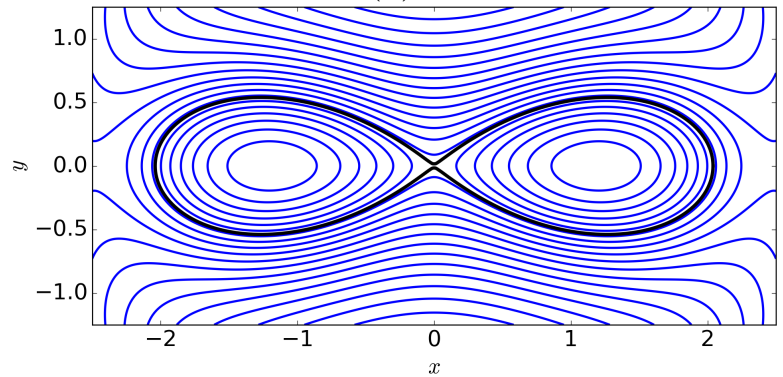

(c)

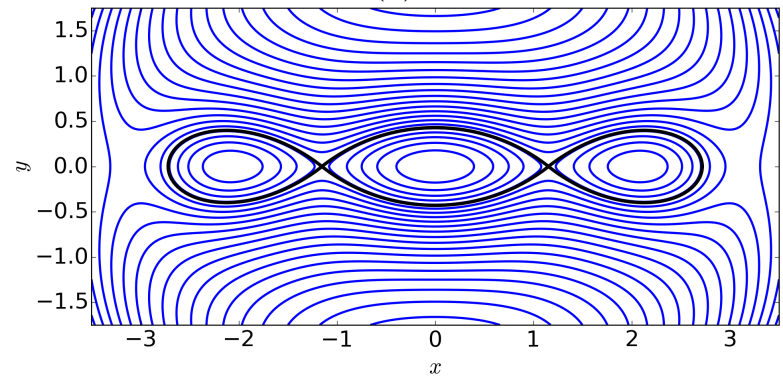

(b)

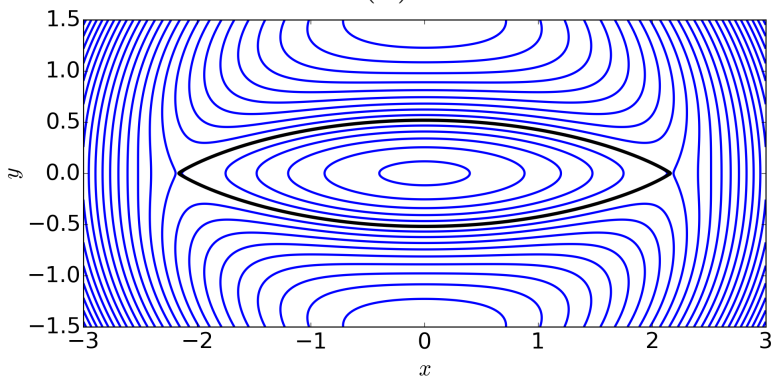

(d)

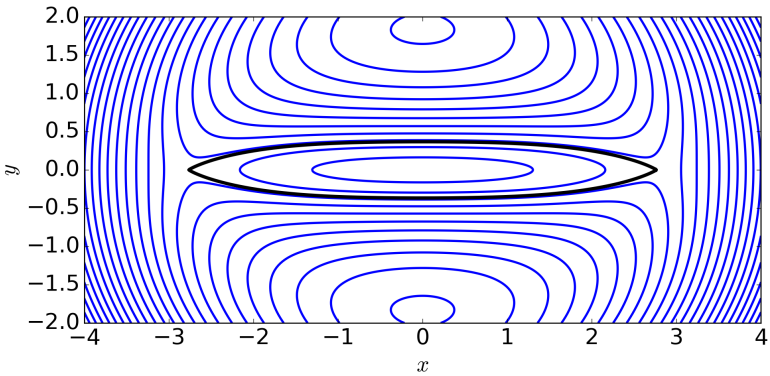

FiguRE 3. Form of the (near) limiting solutions (black contours) and the co-rotating stream function (blue) for (a) the end of the first branch, (b) the start of the second branch, (c) the end of the second branch, and (d) the start of the third branch. Note: 'start' and 'end' correspond to 'right' and 'left' respectively in figures 1 and 2 .

array of three vortices connected at two stagnation points. This pattern continues for the other branches, with the vortex become increasingly elongated. The (near) limiting states are illustrated in figures 3 and 4 together with the stream function in the co-rotating frame of reference.

Key properties of the two-fold limiting states for $\varepsilon=0.01$ are provided in Table 1.

3.2. 3-fold vortex patch equilibria. We next turn to 3-fold symmetric vortex patch equilibria, first studied by Deem and Zabusky in 1978 [6] for the Euler equations $(\varepsilon=0)$. Here we discuss the structure of the solution branches for a wide range of $\varepsilon$.

The most surprising result is that there is a disconnected branch of solutions, not terminating at either end at the circular Rankine vortex. This was discovered by increasing $\varepsilon$ and finding a change in the topology of the limiting states between $\varepsilon=3.5$ and 3.6. This is associated with a bifurcation in the structure of the solution branches, as shown in figure 5 . For $\varepsilon=3.5$ (black curves), the branch starting from the circular patch in the upper right corner reaches a minimum in $\Omega$ at $\Omega=0.007704627$, then increases and finally decreases approaching the limiting state. (In fact $\mathrm{d} \Omega / \mathrm{d} \Omega_{p}$ likely changes sign an infinite number of times before reaching the limiting state at $\Omega_{p}=0$.) This limiting state is triangular (albeit with curved sides), and has the same form as found for the Euler equations $(\varepsilon=0)$ in [6] (see below). For $\varepsilon=3.6$ (blue curves), the branch starting from the circular patch in the upper right corner also reaches a minimum in $\Omega$, but then $\Omega$ increases and limits to a significantly smaller value as $\Omega_{p} \rightarrow 0$. This limiting state resembles three petal-like vortices connected at a single point at the origin. This state is also the limiting state of 
(a)

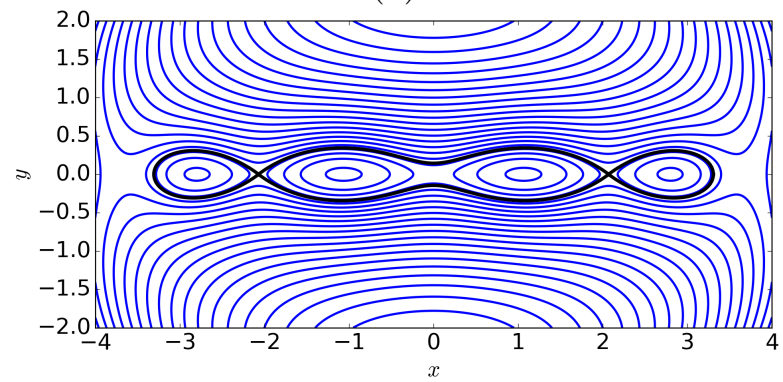

(c)

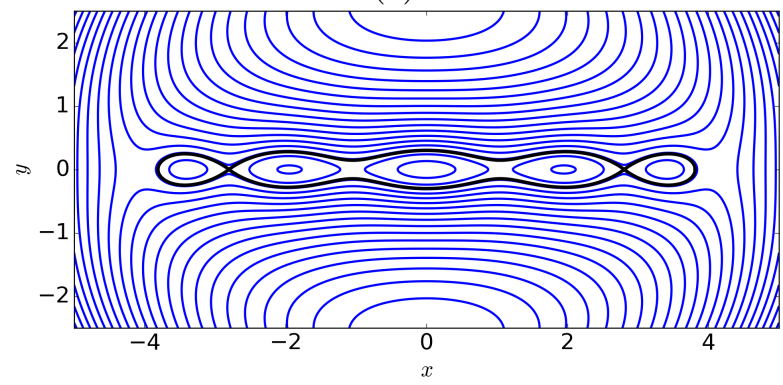

(b)

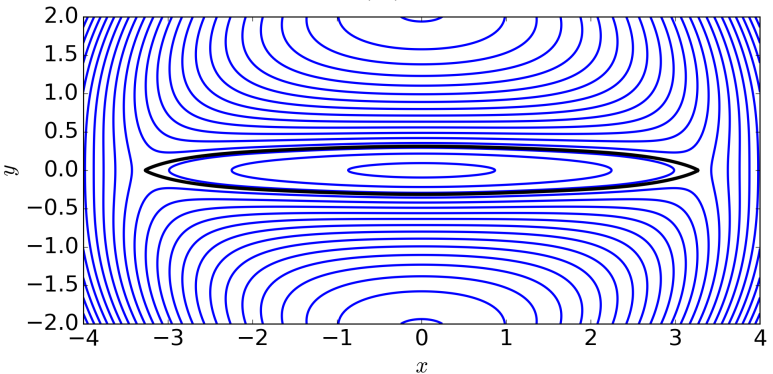

(d)

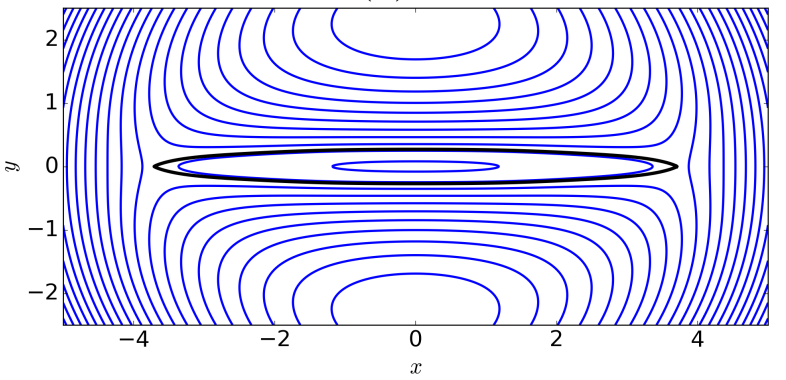

Figure 4. Form of the (near) limiting solutions (black contours) and the co-rotating stream function (blue) for (a) the end of the third branch, (b) the start of the fourth branch, (c) the end of the fourth branch, and (d) the start of the fifth branch.

\begin{tabular}{|c||c|c|r|}
\hline \multicolumn{1}{|c|}{ Branch } & $\Omega$ & $\pi / 2 J$ & \multicolumn{1}{|c|}{$16 E / \pi$} \\
\hline $1 \mathrm{a}$ & 0.250000 & 1.000000 & 1.000000 \\
$1 \mathrm{~b}$ & 0.106827 & 0.317246 & -0.456852 \\
$2 \mathrm{a}$ & 0.159754 & 0.471293 & 0.110399 \\
$2 \mathrm{~b}$ & 0.076297 & 0.195486 & -1.231673 \\
$3 \mathrm{a}$ & 0.107035 & 0.272772 & -0.692599 \\
$3 \mathrm{~b}$ & 0.059980 & 0.142097 & -1.777402 \\
$4 \mathrm{a}$ & 0.080209 & 0.191364 & -1.268668 \\
$4 \mathrm{~b}$ & 0.049782 & 0.113129 & -2.181805 \\
$5 \mathrm{a}$ & 0.064135 & 0.147404 & -1.714846 \\
$5 \mathrm{~b}$ & 0.042700 & 0.094682 & -2.504112 \\
\hline
\end{tabular}

TABLE 1. Key properties of the (near) limiting states for $\varepsilon=0.01$ for the first 5 branches shown in figure 1 . In the first column, 'a' denotes the start of a branch while ' $b$ ' denotes the end of it.

three identical co-rotating vortex patches, first studies in [10]. Having determined that there is a bifurcation in the solution branch structure between $\varepsilon=3.5$ and 3.6, a new branch was discovered by taking the near limiting solution for $\varepsilon=3.6$ and gradually decreasing $\varepsilon$ to $\varepsilon=3.5$, holding the angular impulse fixed ( $\Omega$ must be allowed to vary). In this way, we could find a solution on the black separated branch next to the blue one (the middle pair of curves in the lower part of the figure with $\Omega \approx 0.007$ ). Having found one solution, we could then continue in both directions 


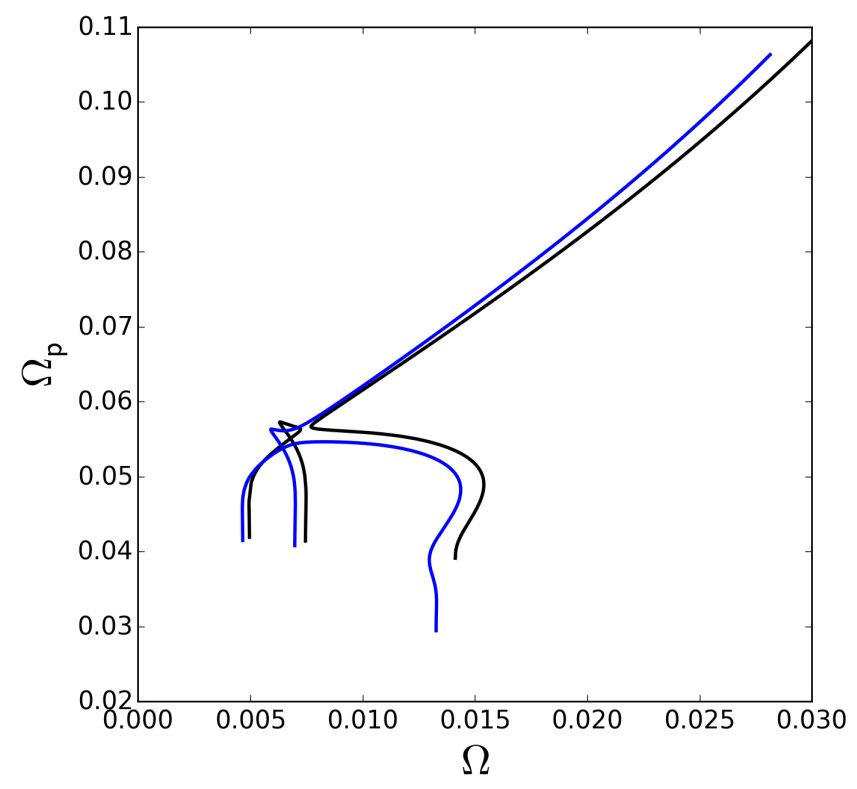

FiguRE 5. Solution branch structure for 3-fold vortex patch equilibria when $\varepsilon=3.5$ (black) and $\varepsilon=3.6$ (blue). The branches start at the circular vortex patch solution in the upper right portion of the graph.

(a)

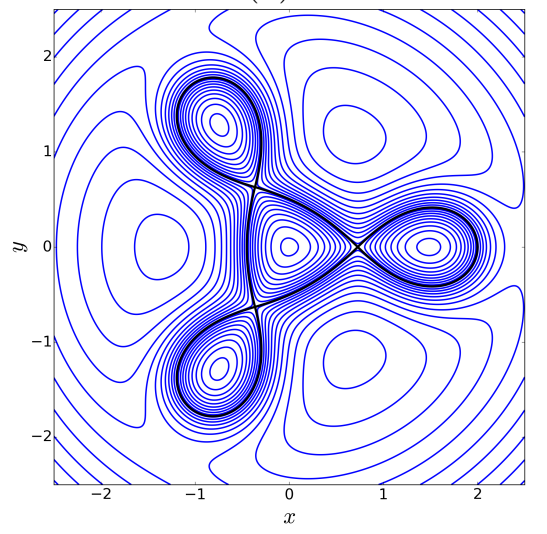

(b)

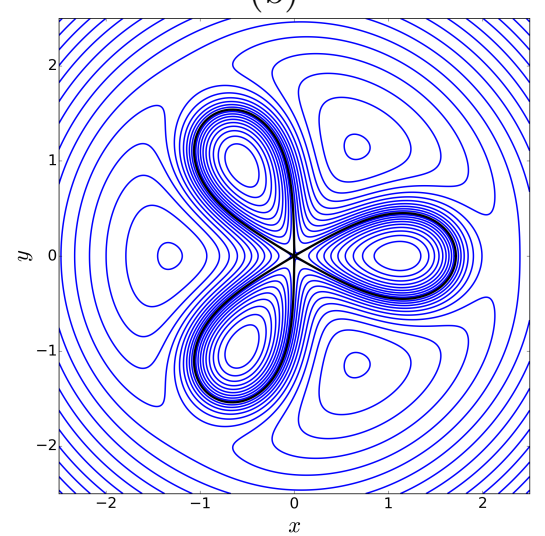

(c)

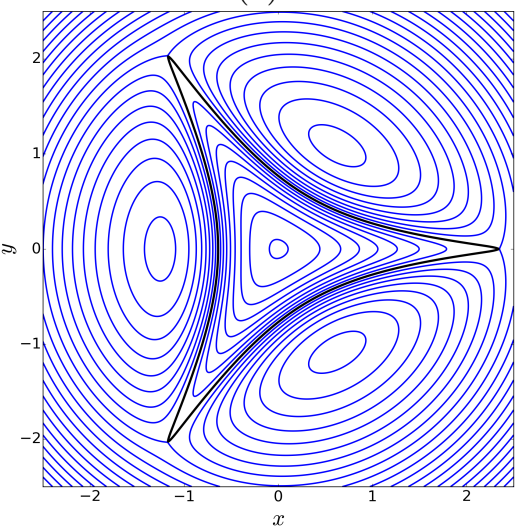

Figure 6. Form of the (near) limiting solutions (black contours) and the co-rotating stream function (blue) for the state with (a) the smallest $\Omega$, (b) the intermediate value of $\Omega$, and (c) the largest $\Omega$. Here $\varepsilon=3.6$, corresponding to the blue curves in figure 5 .

to find the limiting states on this separated branch. (The blue separated branch can be found similarly by jumping from the black separated branch at small $\Omega$.) One of these limiting states is the petal-like state just described. The other, at the smallest $\Omega$, is a new state consisting of a triangular central vortex attached to three petals - a four vortex state. These limiting solutions are illustrated for $\varepsilon=3.6$ in figure 6 . 


\begin{tabular}{|c|c|r|}
\hline$\Omega$ & $\pi / 2 J$ & \multicolumn{1}{c|}{$16 E / \pi$} \\
\hline 0.333333 & 1.000000 & 1.000000 \\
0.301234 & 0.885055 & 0.835043 \\
0.122420 & 0.290564 & -1.002261 \\
0.113192 & 0.272230 & -1.110795 \\
\hline
\end{tabular}

TABLE 2. Key properties of the three-fold (near) limiting states for $\varepsilon=0$.

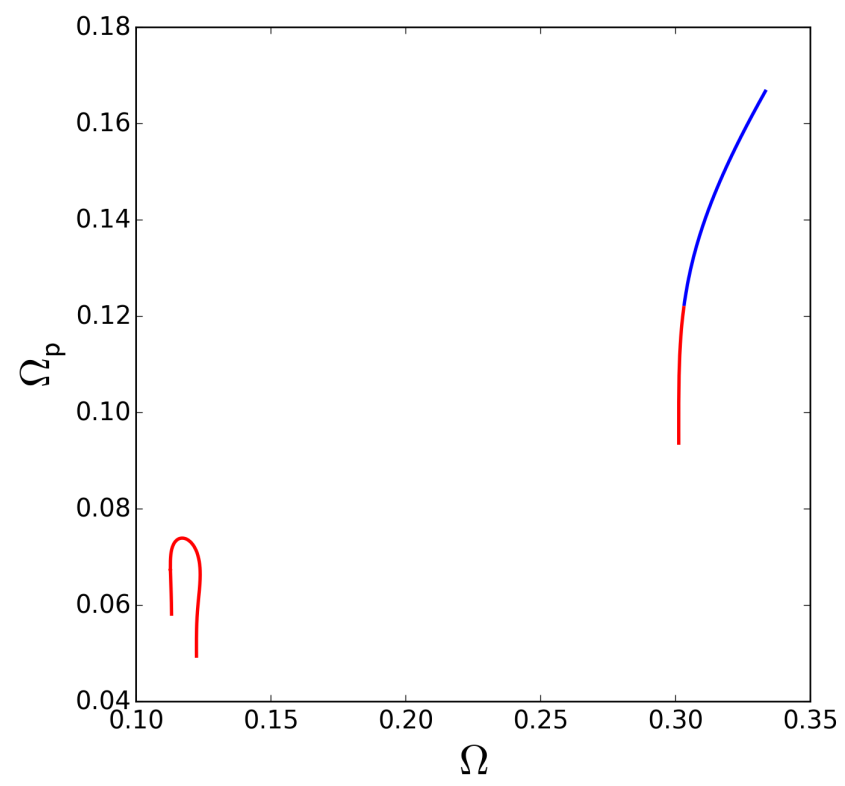

FigURE 7 . Solution branch structure for 3 -fold vortex patch equilibria when $\varepsilon=$ 0 (the Euler equations). The primary branch starts at the circular vortex patch solution in the upper right portion of the graph. There, $\Omega=\frac{1}{3}$ and $\Omega_{p}=\frac{1}{6}$. The separated branch is new. The blue portion of the primary branch is linearly stable while the red portion and the entire separated branch is linearly unstable.

By continuing to jump branches from one value of $\varepsilon$ to another, we were able to determine that separated branches exist over a wide range of $\varepsilon$, and likely for all $\varepsilon$. As $\varepsilon \rightarrow 0$, the separated branch moves far from the primary branch stemming from the circular patch, as shown in figure 7. The limiting states are qualitatively similar to those for $\varepsilon=3.6$ and are shown in figure 8 . Key properties of the three-fold limiting states for $\varepsilon=0$ are provided in Table 2 .

The limiting solutions all appear to exhibit corners on their boundaries, though it is impossible to accurately resolve these corners with the numerical algorithm developed. Zooms of a small portion of the near-limiting solutions in figures $8(\mathrm{a}-\mathrm{c})$ are shown in figures $9(\mathrm{a}-\mathrm{c})$ respectively. Both (a) and (c) appear to limit to right-angled corners, consistent with the analysis of Overman (1986) [22], who demonstrated that the tangent angle through an isolated corner as in (c) must turn by $90^{\circ}$, and that this result also holds for doubly-connected states close to that in (a). The situation in (b) is different. This is close to the limiting triply-connected state found in [10], and the corner angle (in the sectors of rotational fluid) depends on the rotation rate of the equilibrium. [22] estimates 
(a)

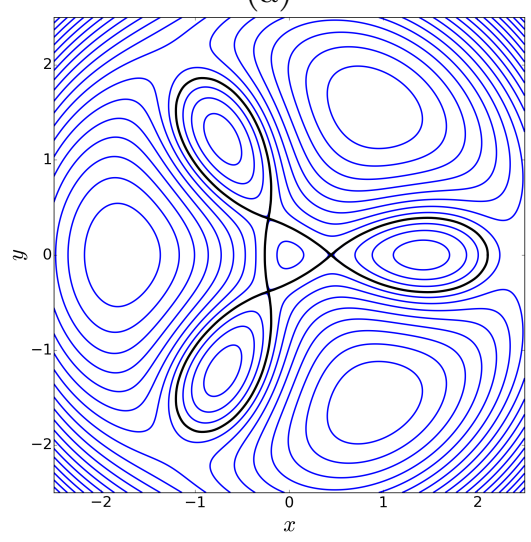

(b)

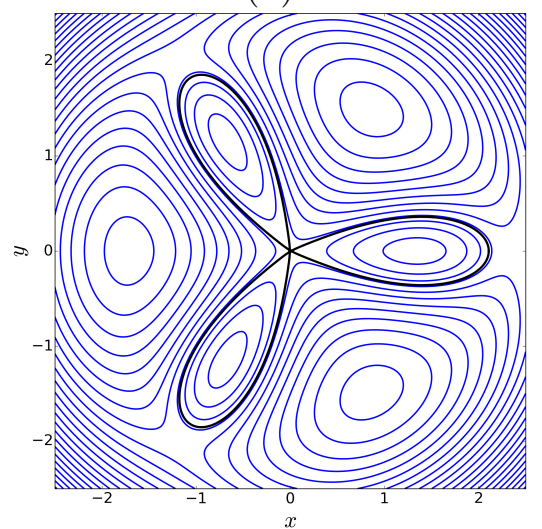

(c)

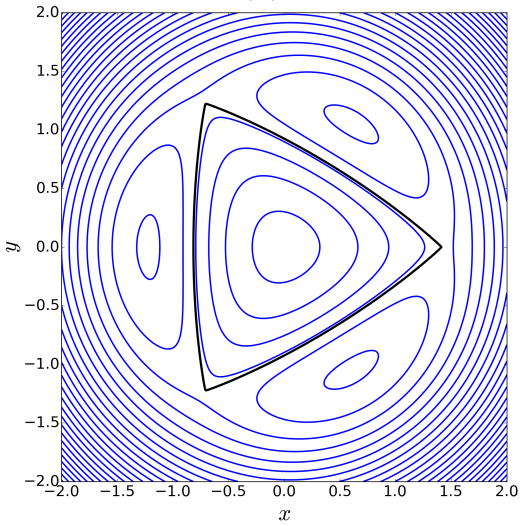

FIgURE 8. Form of the (near) limiting solutions (black contours) and the co-rotating stream function (blue) for the state with (a) the smallest $\Omega$, (b) the intermediate value of $\Omega$, and (c) the largest $\Omega$. Here $\varepsilon=0$, corresponding to the Euler equations. The state in (c) at the end of the primary branch of solutions was discovered in [6]; the state in (b) was discovered in [10], while that in (a) is new.

(a)

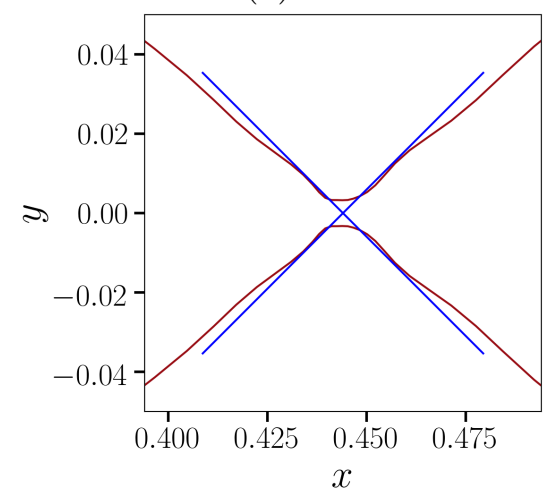

(b)

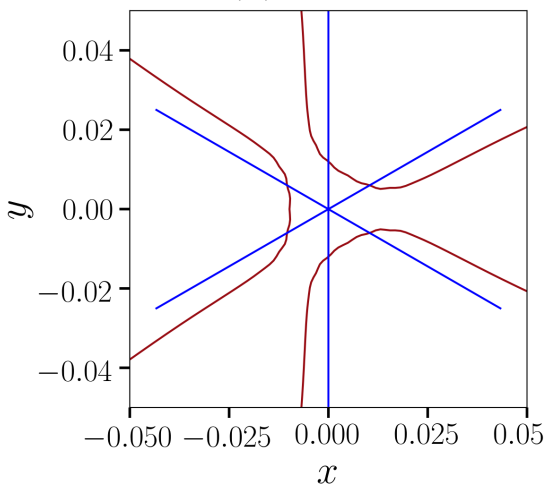

(c)

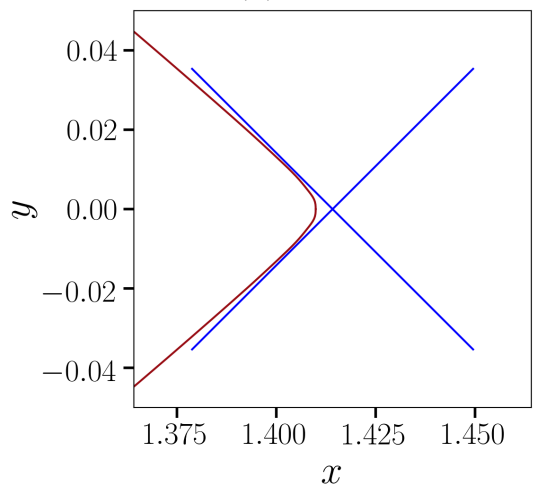

FigURE 9. Zooms showing the vortex boundaries corresponding to the near-limiting states exhibited in figures $8(\mathrm{a}-\mathrm{c})$. The additional blue lines in (a) and (c) cross at $90^{\circ}$, while those in (b) cross at $60^{\circ}$. In (a), the image is centred at $(x, y)=(0.444,0)$, while in $(\mathrm{c})$ it is centred at $(\sqrt{2}, 0)$.

this angle to be approximately $46^{\circ}$ (using $\Omega=0.122420$ from Table 2 , the angle is found to be closer to $\left.46.9^{\circ}\right)$. The evidence in figures $9(\mathrm{~b})$ is however inconclusive.

A more complete picture of the bifurcation structure of the three-fold patch solutions for various values of $\varepsilon$ is provided in figure 10. Each value of $\varepsilon$ is seen to have a separated branch which exists at values of $\Omega$ smaller than that of the limiting circular patch solution. In all cases, this separated branch is linearly unstable. Portions of the primary branch are also unstable, though there is a window of stability for all $\varepsilon>0$. The nonlinear evolution of the unstable states is deferred to another study, but the instabilities often leave a time-dependent pulsating state. 


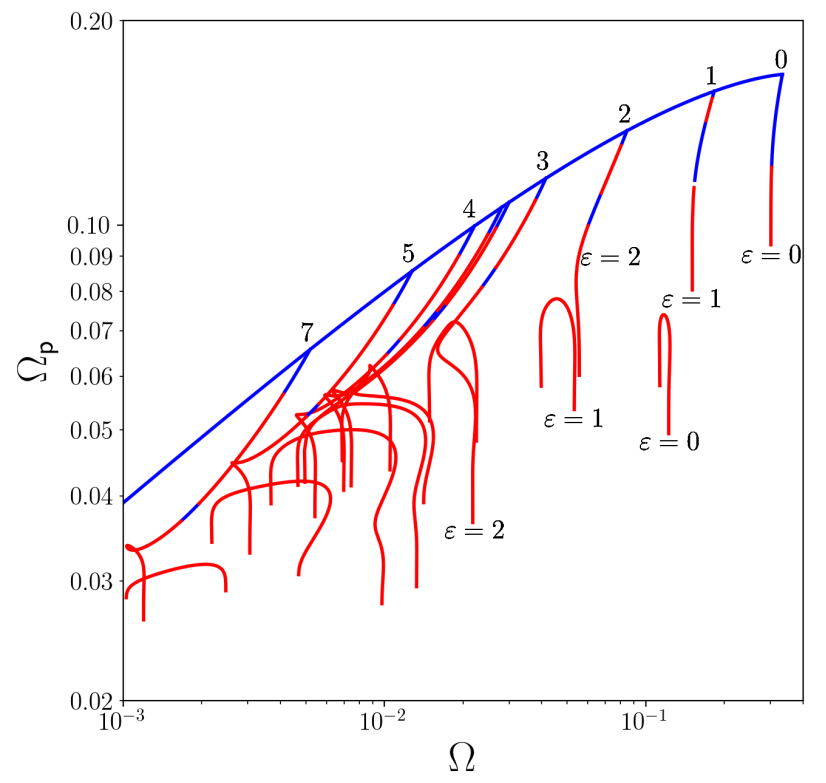

FiguRE 10. Solution branch structure for 3-fold vortex patch equilibria for various $\varepsilon$, as labelled. Blue portions of the curves are linearly stable while red portions are linearly unstable, as determined by a full linear stability analysis (following [10] and [23]). The uppermost blue curve connects the limiting circular patch solutions continuously as a function of $\varepsilon$. Note: small gaps between the blue and red portions of the curves appear because successive equilibria are separated by non-zero increments in $\Omega$ or $J$. Thus gaps occur where there is a change in stability.

\section{TOOLS USED FOR THE MATHEMATICAL ANALYSIS}

The purpose of this section is to review and collect some technical tools that are used throughout the remainder of this paper. We first recall some simple facts about Hölder spaces on the unit circle. Second, we discuss basic properties of modified Bessel functions. Last we state the classical Crandall-Rabinowitz theorem and give a generalized version with a parameter.

4.1. Notation. Here we introduce some notation that is used in the forthcoming sections.

- The unit disc of the plane and its boundary will be denoted by $\mathbb{D}$ and $\mathbb{T}$, respectively.

- We denote by $C$ any positive constant that may change from line to line.

- For a given continuous function $f: \mathbb{T} \rightarrow \mathbb{C}$, we define its mean value by

$$
f_{\mathbb{T}} f(\tau) d \tau \triangleq \frac{1}{2 i \pi} \int_{\mathbb{T}} f(\tau) d \tau
$$

where $d \tau$ stands for the complex integration.

- Let $X$ and $Y$ be two normed spaces. We denote by $\mathcal{L}(X, Y)$ the vector space of all the continuous linear maps endowed with its usual strong topology.

- Let $Y$ be a vector space and $R$ be a subspace, then $Y / R$ denotes the quotient space.

4.2. Modified Bessel functions. This section is devoted to some classical properties of Bessel functions of imaginary argument. We start with the Bessel function of the first kind and order $\nu$ 
given by the expansion

$$
J_{\nu}(z)=\sum_{m=0}^{+\infty} \frac{(-1)^{m}\left(\frac{z}{2}\right)^{\nu+2 m}}{m ! \Gamma(\nu+m+1)}, \quad|\arg (z)|<\pi .
$$

Note that this sum converges in a classical way provided that $\Gamma(\nu+m+1)$ exists for any positive integer. In addition, for $\nu=n \in \mathbb{Z}$ it is known that Bessel functions admit the following integral representation:

$$
\forall z \in \mathbb{C}, \quad J_{n}(z)=\frac{1}{\pi} \int_{0}^{\pi} \cos (n \theta-z \sin \theta) d \theta .
$$

Bessel functions of imaginary argument, denoted by $I_{\nu}$ and $K_{\nu}$, are given by

$$
I_{\nu}(z)=\sum_{m=0}^{+\infty} \frac{\left(\frac{z}{2}\right)^{\nu+2 m}}{m ! \Gamma(\nu+m+1)}, \quad|\arg (z)|<\pi
$$

and

$$
K_{\nu}(z)=\frac{\pi}{2} \frac{I_{-\nu}(z)-I_{\nu}(z)}{\sin (\nu \pi)}, \nu \in \mathbb{C} \backslash \mathbb{Z} \quad|\arg (z)|<\pi .
$$

However, for $\nu=n \in \mathbb{Z}$ we set $K_{n}(z)=\lim _{\nu \rightarrow n} K_{\nu}(z)$. At this stage we recall useful expansions for $K_{n}$ that can be found for instance in [29, p. 79-80],

$$
K_{0}(z)=-\log \left(\frac{z}{2}\right) I_{0}(z)+\sum_{m=0}^{\infty} \frac{\left(\frac{z}{2}\right)^{2 m}}{(m !)^{2}} \psi(m+1), \quad K_{0}^{\prime}(z)=-K_{1}(z)
$$

where

$$
\psi(1)=-\gamma \quad \text { and } \quad \forall m \in \mathbb{N}^{*}, \quad \psi(m+1)=\sum_{k=1}^{m} \frac{1}{k}-\gamma .
$$

In addition, for $n \in \mathbb{N}^{*}$

$$
\begin{aligned}
K_{n}(z)= & (-1)^{n+1} \sum_{m=0}^{+\infty} \frac{\left(\frac{z}{2}\right)^{n+2 m}}{m !(n+m) !}\left(\log \left(\frac{z}{2}\right)-\frac{1}{2} \psi(m+1)-\frac{1}{2} \psi(n+m+1)\right) \\
& +\frac{1}{2} \sum_{m=0}^{n-1} \frac{(-1)^{m}(n-m-1) !}{m !\left(\frac{z}{2}\right)^{n-2 m}} .
\end{aligned}
$$

Another useful property is the positivity of $I_{n}$ and $K_{n}$. In fact, for any $n \in \mathbb{N}$ we have

$$
\forall x>0, I_{n}(x)>0 \text { and } K_{n}(x)>0 .
$$

The first one is obvious from the definition since each term in the sum is strictly positive. As for the second one, it can be deduced from the following integral representation found in e.g. [29, p. $181]$

$$
K_{\nu}(z)=\int_{0}^{+\infty} e^{-z \cosh t} \cosh (\nu t) d t .
$$

Another useful identity found in [29, p. 441] deals with Nicholson's integral representation of $I_{n}(z) K_{n}(z):$ for $n \in \mathbb{N}$

$$
I_{n}(z) K_{n}(z)=\frac{2(-1)^{n}}{\pi} \int_{0}^{\frac{\pi}{2}} K_{0}(2 z \cos \theta) \cos (2 n \theta) d \theta .
$$


For the convenience of the reader, we next describe the relationship between $K_{0}$ and the Green function associated with Helmholtz's operator in two-dimensional space. Let $\varepsilon \in \mathbb{R}^{*}$ and consider in the distribution sense the equation

$$
\left(-\Delta+\varepsilon^{2}\right) G_{\varepsilon}=\delta_{0}, \text { in } \quad \mathcal{S}^{\prime}\left(\mathbb{R}^{2}\right)
$$

Then using a Fourier transform we obtain

$$
\widehat{G}_{\varepsilon}(\xi)=\frac{1}{|\xi|^{2}+\varepsilon^{2}}, \quad \forall \xi \in \mathbb{R}^{2} .
$$

Thus by a scaling argument, we have

$$
G_{\varepsilon}(x)=G_{1}(\varepsilon x), \quad \text { with } \quad G_{1}(x)=\frac{1}{4 \pi^{2}} \int_{\mathbb{R}^{2}} \frac{e^{i x \cdot \xi}}{1+|\xi|^{2}} d \xi .
$$

Hence, a change of variables using polar coordinates yields

$$
\begin{aligned}
G_{1}(x) & =\frac{1}{4 \pi^{2}} \int_{0}^{+\infty} \frac{r}{1+r^{2}} \int_{0}^{2 \pi} \cos (|x| r \cos \theta) d \theta d r \\
& =\frac{1}{2 \pi} \int_{0}^{+\infty} \frac{r J_{0}(|x| r)}{1+r^{2}} d r \\
& =\frac{1}{2 \pi} K_{0}(|x|),
\end{aligned}
$$

where in the last line we have used an identity from [29, p. 425]. As an application we show how to recover the velocity from the domain of the patch in (1.1). In fact, if $D$ is a smooth bounded simply-connected domain and $q=\mathbf{1}_{D}$, then from the foregoing results the stream function $\psi$, which is the solution of the elliptic equation

$$
\left(\Delta-\varepsilon^{2}\right) \psi=\mathbf{1}_{D}
$$

is given explicitly by

$$
\psi(x)=-\frac{1}{2 \pi} \int_{\mathbb{R}^{2}} K_{0}(|\varepsilon||x-y|) \mathbf{1}_{D}(y) d A(y)
$$

where $d A$ denotes the planar Lebesgue measure. It follows that the velocity induced by the patch $v=\nabla^{\perp} \psi$ takes the form

$$
v(x)=\frac{1}{2 \pi} \int_{\partial D} K_{0}(|\varepsilon||x-\xi|) d \xi,
$$

where the integration should be understood in the complex sense.

4.3. Boundary equations. In what follows we state the boundary equation of a rotating patch. First, the initial data $q_{0}=\mathbf{1}_{D}$ generate a rotating patch about the origin with uniform angular velocity $\Omega \in \mathbb{R}$ if

$$
q(t)=\mathbf{1}_{D_{t}} \quad \text { with } \quad D_{t}=e^{i t \Omega} D .
$$

We may check that this is equivalent to

$$
\left(v(x)-\Omega x^{\perp}\right) \cdot \vec{n}(x)=0, \quad \forall x \in \partial D
$$

with $\vec{n}(x)$ being the unit outward normal vector to the boundary at the point $x$. The velocity $v$ induced by $q_{0}$ is given by (4.5). Using complex notation we find that

$$
\forall w \in \mathbb{T}, G(\varepsilon, \Omega, \Phi)(w)=0
$$

with

$$
G(\varepsilon, \Omega, \Phi)(w)=\operatorname{Im}\left\{\Omega \Phi(w) \overline{\Phi^{\prime}(w)} \bar{w}-\overline{\Phi^{\prime}(w)} \bar{w} f_{\mathbb{T}} \Phi^{\prime}(\tau) K_{0}(|\varepsilon||\Phi(w)-\Phi(\tau)|) d \tau\right\}
$$


and $\Phi: \mathbb{T} \rightarrow \mathbb{C}$ is at least a $C^{1}$ parametrization of the boundary. Actually, we may add a constant term in the kernel $K_{0}$ without changing the equation. Thus according to the singularity structure of $K_{0}$ near the origin detailed in (4.1) the suitable constant to add is $\log \left(\frac{|\varepsilon|}{2}\right)$. Therefore

$$
G(\varepsilon, \Omega, \Phi)(w)=\operatorname{Im}\left\{\Omega \Phi(w) \overline{\Phi^{\prime}(w)} \bar{w}-\overline{\Phi^{\prime}(w)} \bar{w} f_{\mathbb{T}} \Phi^{\prime}(\tau) K_{0}^{\varepsilon}(|\Phi(w)-\Phi(\tau)|) d \tau\right\}
$$

with

$$
K_{0}^{\varepsilon}(x) \triangleq K_{0}(|\varepsilon| x)+\log (|\varepsilon| / 2) .
$$

If we let $\varepsilon \rightarrow 0$, then without surprise we get the vortex patch equation associated with the Euler equations described for example in [15]:

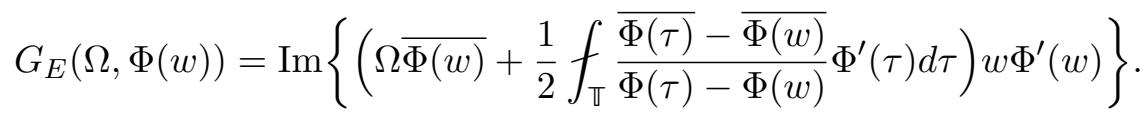

One may notice that

$$
G(0, \Omega, \Phi(w))=-G_{E}(\Omega, \Phi(w)) .
$$

Indeed, starting from the general formula

$$
f_{\partial D} \log |z-\xi| d \xi=-\frac{1}{2} \overline{f_{\partial D} \bar{\xi}-\bar{z}} d \xi
$$

we find by a change of variables

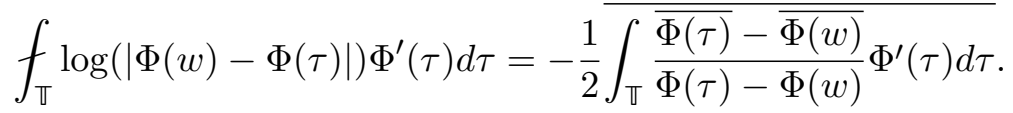

Thus

$$
G_{E}(\Omega, \Phi(w))=-\operatorname{Im}\left\{\left(\Omega \Phi(w)+f_{\mathbb{T}} \log (|\Phi(w)-\Phi(\tau)|) \Phi^{\prime}(\tau) d \tau\right) \bar{w} \overline{\Phi^{\prime}(w)}\right\} .
$$

It suffices now to use the expansion (4.1) to deduce that

$$
\forall x \neq 0, \quad \lim _{\varepsilon \rightarrow 0} K_{0}^{\varepsilon}(x)=-\log (x / 2)
$$

and thus we find (4.7).

\section{Bifurcation to $m$-FOLD SYMMETRIC VORTEX PATCH EQUILIBRIA}

The main task of this section is to prove the existence of rotating m-fold vortex patch (relative) equilibria, or 'V-states' [6], for the QGSW model given by (1.1). In the first section we state our main result. The proof is carried out in several steps and is detailed in different sections. The basic tool is the classical Crandall-Rabinowitz's theorem, and for the study of the imperfect bifurcation we need a slight generalization of this theorem.

5.1. Main result. We first state our principal result concerning the existence of a countable family of bifurcating curves with m-fold symmetry from Rankine (circular) vortices. More precisely, we obtain the following result.

Theorem 5.1. Let $\varepsilon \in \mathbb{R}$, then for each integer $m \geq 1$ there exists a curve (or branch) of $m$-fold rotating vortex patches bifurcating from the unit disc at the angular velocity

$$
\Omega_{m}(\varepsilon)=I_{1}(|\varepsilon|) K_{1}(|\varepsilon|)-I_{m}(|\varepsilon|) K_{m}(|\varepsilon|) .
$$


Moreover, the existence is uniform for vanishing $\varepsilon$. More precisely, there exists $a>0$ and continuous functions $\varphi:(-a, a)^{2} \rightarrow \mathbb{R}, \psi:(-a, a)^{2} \rightarrow C^{1+\alpha}(\mathbb{T})$ satisfying

$$
\varphi(0,0)=\Omega_{m}(0)=\frac{m-1}{2 m}, \quad \psi(0,0)=0
$$

such that

$$
\Omega=\varphi(\varepsilon, s), \quad \psi(\varepsilon, s, w)=\sum_{n \geq 2} a_{n m-1}(\varepsilon, s) \bar{w}^{n m-1}
$$

and

$$
G\left(\varepsilon, \varphi(\varepsilon, s), w+s \bar{w}^{m-1}+s \psi(\varepsilon, s, w)\right)=0, \forall(\varepsilon, s) \in(-a, a)^{2}, \quad \forall w \in \mathbb{T} .
$$

Recall that the function $G$ defining the vortex patch equation is given by (4.6).

Remark 5.2. On the one hand, the case $m=1$ is trivial. It corresponds simply to the translation of the unit disc, and with the notation of the theorem we have $\psi(\varepsilon, s, w)=0$. On the other hand, the regularity of the boundary is not at all optimal; like for the Euler equations we guess that it must be analytic, see [3, 14].

Remark 5.3. It is known that for the Euler equations the bifurcation diagram of simply connected vortex patches is organized around Rankine vortices through a countable collection of pitchfork curves (one for each symmetry). Theorem 5.1 shows that locally this structure is preserved for any perturbation size of $\varepsilon$ and therefore there is no symmetry breakdown. This is not the case however for the bifurcation diagram close to Kirchhoff's ellipse, as discussed below in Section 6.

The proof of Theorem 5.1 is a consequence of the materials developed in next sections. Actually, the first part of Theorem 5.1 follows from Theorem 5.4, Proposition 5.7, and Proposition 5.9. However for the second part dealing with the stability of Eulerian branches under small perturbation on $\varepsilon$, we need to make use of Theorem 5.5.

5.2. Crandall-Rabinowitz's Theorem with a parameter. The main objective of this section is to formulate suitable conditions for the bifurcation from the trivial solutions of a general nonlinear equation of the type

$$
F(\varepsilon, \lambda, x)=0, \quad F: \mathbb{R} \times \mathbb{R} \times V \rightarrow Y
$$

with $Y$ a Banach space and $V$ a neighborhood of 0 in some Banach space $X$. We assume that $F$ is smooth enough and

$$
\forall \lambda, F(0, \lambda, 0)=0 .
$$

The starting point is that for $\varepsilon=0$ we know the structure of the bifurcation diagram near the trivial solutions and it is of interest to understand how its geometric structure varies with respect to an arbitrary perturbation in $\varepsilon$. This is called an imperfect bifurcation. This subject is well developed in the literature starting with the pioneering work of Golubitsky and Schaeffer [11], who classify the bifurcation diagram in a general setting using tools from the theory of singularities. Various particular studies related to the present study have been carried out over the last few decades, and one may consult for instance $[18,27]$ and the references therein. In what follows we formulate some results dealing with imperfect bifurcations with symmetry persistence. This phenomenon occurs especially when the trivial solutions do not vary with respect to the parameter $\varepsilon$, that is

$$
\forall \lambda, \forall \varepsilon, F(\varepsilon, \lambda, 0)=0 .
$$

For the steadily-rotating vortex patch solutions of (1.1), these are precisely the suitable conditions enabling a detailed study of the bifurcations from the unit disc. Next we recall the classical theorem of Crandall-Rabinowitz [5] concerning bifurcations from trivial solutions. This will be applied to get the first part of Theorem 5.1 when $\varepsilon$ is fixed at an arbitrary value. 
Theorem 5.4. Let $X, Y$ be two Banach spaces, $V$ be a neighborhood of 0 in $X$ and let

$$
F: \mathbb{R} \times V \rightarrow Y
$$

with the following properties:

(1) $F(\lambda, 0)=0$ for any $\lambda \in \mathbb{R}$.

(2) The partial derivatives $F_{\lambda}, F_{x}$ and $F_{\lambda x}$ exist and are continuous.

(3) $\operatorname{Ker}\left(\partial_{x} F(0,0)\right)=\left\langle x_{0}\right\rangle$ and $Y / R\left(\partial_{x} F(0,0)\right)$ are one-dimensional.

(4) Transversality assumption: $\partial_{\lambda} \partial_{x} F(0,0) x_{0} \notin R\left(\partial_{x} F(0,0)\right)$.

If $\mathcal{X}$ is any complement of $\operatorname{Ker}\left(\partial_{x} F(0,0)\right)$ in $X$, then there is a neighborhood $U$ of $(0,0)$ in $\mathbb{R} \times X$, an interval $(-a, a)$, and continuous functions $\psi:(-a, a) \rightarrow \mathbb{R}, \phi:(-a, a) \rightarrow Z$ such that $\psi(0)=0$, $\phi(0)=0$ and

$$
\{(\lambda, x) \in U, F(\lambda, x)=0\}=\left\{\left(\psi(s), s x_{0}+s \phi(s)\right) ;|s|<a\right\} \cup\{(\lambda, 0) ;(\lambda, 0) \in U\} .
$$

The next result deals with a slight generalization of the preceding Crandall-Rabinowitz's theorem to include a parameter. This allows us to treat the stability of the bifurcation diagram under a small perturbation. This will be the cornerstone of the proof of the second part of Theorem 5.1.

Theorem 5.5. Let $X, Y$ be two Banach spaces, $V$ a neighbourhood of 0 in $X$ and let

$$
F:(-1,1) \times \mathbb{R} \times V \rightarrow Y
$$

be a function of class $C^{1}$ with the following properties:

(1) $F(\varepsilon, \lambda, 0)=0$ for any $\varepsilon \in \mathbb{R}$ and $\lambda \in \mathbb{R}$.

(2) The partial derivatives $F_{\varepsilon}, F_{\lambda}, F_{x}$ and $F_{\lambda x}$ exist and are continuous.

(3) $\operatorname{Ker}\left(\partial_{x} F(0,0,0)\right)=\left\langle x_{0}\right\rangle$ and $Y / R\left(\partial_{x} F(0,0,0)\right)$ are one-dimensional.

(4) Transversality assumption: $\partial_{\lambda} \partial_{x} F(0,0,0) x_{0} \notin R\left(\partial_{x} F(0,0,0)\right)$.

If $\mathcal{X}$ is any complement of $\operatorname{Ker}\left(\partial_{x} F(0,0,0)\right)$ in $X$, then there is a neighborhood $U$ of $(0,0,0)$, an interval $(-a, a)$, with $a>0$, and continuous functions

$$
\psi:(-a, a)^{2} \rightarrow \mathbb{R}, \quad \phi:(-a, a)^{2} \rightarrow \mathcal{X}
$$

such that $\varphi(0,0)=0, \psi(0,0)=0$ and

$\{(\varepsilon, \lambda, x) \in U, F(\varepsilon, \lambda, x)=0\}=\left\{\left(\varepsilon, \psi(\varepsilon, s), s x_{0}+s \phi(\varepsilon, s)\right) ;|\varepsilon|,|s|<a\right\} \cup\{(\varepsilon, \lambda, 0) ;(\varepsilon, \lambda, 0) \in U\}$.

Proof. The proof is a simple adaptation of [5]. Let $\left\langle w_{0}\right\rangle$ be a complement of $\mathcal{Y} \triangleq \mathcal{R}\left(\partial_{x} F(0,0,0)\right)$ in $Y$. Then

$$
X=\left\langle x_{0}\right\rangle \oplus \mathcal{X} \quad \text { and } \quad Y=\left\langle w_{0}\right\rangle \oplus \mathcal{Y} .
$$

Consider the projection $P: X \mapsto\left\langle x_{0}\right\rangle$ on $\left\langle x_{0}\right\rangle$ along $\mathcal{X}$ given by

$$
x=s x_{0}+z, z \in \mathcal{X} \Longrightarrow P x=s x_{0}
$$

and similarly define the projection $Q: Y \mapsto\left\langle w_{0}\right\rangle$ on $\left\langle w_{0}\right\rangle$ along $\mathcal{Y}$. Then the equation $F(\varepsilon, \lambda, x)=0$ is equivalent to the system

$$
F_{1}(\varepsilon, \lambda, s, z) \triangleq(\mathrm{Id}-Q) F\left(\varepsilon, \lambda, s x_{0}+z\right)=0 \quad \text { and } \quad Q F\left(\varepsilon, \lambda, s x_{0}+z\right)=0 .
$$

It is clear that for some $\eta>0$, the function

$$
F_{1}:(-1,1) \times \mathbb{R} \times(-\eta, \eta) \times \mathcal{U} \rightarrow \mathcal{Y}_{m}
$$

is $C^{1}$ with $\mathcal{U}$ a small neighbourhood of 0 in $\mathcal{X}$. Moreover, it is not difficult to check that

$$
\partial_{z} F_{1}(0,0,0,0)=(\operatorname{Id}-Q) \partial_{x} F(0,0,0): \mathcal{X} \rightarrow \mathcal{Y}
$$


is an isomorphism. By the implicit function theorem, the solutions of the equation $F_{1}(\varepsilon, \lambda, s, z)=0$ are described near the point $(0,0,0,0)$ by the parametrization $z=\varphi(\varepsilon, \lambda, s)$ with

$$
\varphi:(-\delta, \delta)^{3} \rightarrow \mathcal{X}, \quad \delta>0
$$

being a $C^{1}$ function. Therefore we obtain

$$
(\operatorname{Id}-Q) F\left(\varepsilon, \lambda, s x_{0}+\varphi(\varepsilon, \lambda, s)\right)=0, \forall|\varepsilon|,|\lambda|,|s|<\delta .
$$

Consequently, solving the equation $F(\varepsilon, \lambda, x)=0$ close to $(0,0,0)$ is equivalent to

$$
F_{2}(\varepsilon, \lambda, s) \triangleq Q F\left(\varepsilon, \lambda, s x_{0}+\varphi(\varepsilon, \lambda, s)\right)=0, \quad \forall|\varepsilon|,|\lambda|,|s|<\delta .
$$

Using the assumption $F(\varepsilon, \lambda, 0)=0$, for any $\varepsilon \in \mathbb{R}$ and $\lambda \in \mathbb{R}$, one deduces by uniqueness that

$$
\varphi(\varepsilon, \lambda, 0)=0, \quad \forall|\varepsilon|,|\lambda|<\delta .
$$

Now differentiating equation (5.1) with respect to $s$ we get

$$
\partial_{s}\left((\operatorname{Id}-Q) F\left(\varepsilon, \lambda, s x_{0}+\varphi(\varepsilon, \lambda, s)\right)\right)=0, \forall|\varepsilon|,|\lambda|,|s|<\delta .
$$

In particular we deduce for $s=0$

$$
(\operatorname{Id}-Q) \partial_{x} F(\varepsilon, \lambda, \varphi(\varepsilon, \lambda, 0))\left(x_{0}+\partial_{s} \varphi(\varepsilon, \lambda, 0)\right)=0
$$

which implies, in view of $(5.2)$, that

$$
\partial_{x} F(0,0,0)\left(\partial_{s} \varphi(0,0,0)\right)=0 .
$$

This gives $\partial_{s} \varphi(0,0,0) \in\left\langle x_{0}\right\rangle$, but from the definition one has $\partial_{s} \varphi(0,0,0) \in \mathcal{X}$ and consequently

$$
\partial_{s} \varphi(0,0,0)=0 \text {. }
$$

Hence there exists a continuous function $\varphi_{1}:(-\delta, \delta)^{3} \rightarrow \mathcal{X}$ such that

$$
\varphi(\varepsilon, \lambda, s)=s \varphi_{1}(\varepsilon, \lambda, s) \text { and } \varphi_{1}(0,0,0)=0 .
$$

Set

$$
g(\varepsilon, \lambda, s) \triangleq\left\{\begin{array}{l}
Q F\left(\varepsilon, \lambda, s x_{0}+\varphi(\varepsilon, \lambda, s)\right) / s, \quad s \neq 0 \\
Q \partial_{x} F(\varepsilon, \lambda, 0)\left[x_{0}+\partial_{s} \varphi(\varepsilon, \lambda, 0)\right], \quad s=0 .
\end{array}\right.
$$

Note that $g$ is continuous and

$$
g(0,0,0)=Q \partial_{x} F(0,0,0) x_{0}=0 .
$$

Moreover, thanks to (5.2) one may easily check that

$$
\partial_{\lambda} \varphi(\varepsilon, \lambda, 0)=0, \forall|\varepsilon|,|\lambda|<\delta .
$$

Consequently, the partial derivative $\partial_{\lambda} g$ exists, it is continuous and satisfies

$$
\partial_{\lambda} g(0,0,0)=Q \partial_{\lambda} \partial_{x} F(0,0,0) x_{0} .
$$

From the transversality assumption we find

$$
\partial_{\lambda} g(0,0,0) \neq 0 \text {. }
$$

Hence we can use a weak version of the implicit function theorem, see Appendix A in [5], and thus find that the solutions of $g(\varepsilon, \lambda, s)=0$ near the origin are parametrized by a $C^{1}$ surface $\psi:(-a, a)^{2} \rightarrow \mathbb{R}$ such that $\lambda=\psi(\varepsilon, s)$ and

$$
g(\varepsilon, \psi(\varepsilon, s), s)=0, \forall|\varepsilon|,|s|<a, a>0 .
$$

Therefore the non-trivial solutions of the equation $F(\varepsilon, \lambda, x)=0$ near the origin are parametrized by

$$
\lambda=\psi(\varepsilon, s), \quad x=s x_{0}+s \varphi_{1}(\varepsilon, \psi(\varepsilon, s), s) \triangleq s x_{0}+s \phi(\varepsilon, s), \quad \forall|s|,|\varepsilon|<a .
$$

This completes the proof of the desired result. 
5.3. Function spaces I. In this section we introduce the function spaces used below in studying the bifurcation from the unit disc. For $\alpha \in(0,1)$, we set

$$
X=\left\{f \in C^{1+\alpha}(\mathbb{T}), \text { s.t. } \forall w \in \mathbb{T}, f(w)=\sum_{n=0}^{+\infty} f_{n} \bar{w}^{n}, f_{n} \in \mathbb{R}\right\}
$$

and

$$
Y=\left\{g \in C^{\alpha}(\mathbb{T}), \text { s.t. } \forall w \in \mathbb{T}, g(w)=\sum_{n=1}^{+\infty} g_{n} e_{n}(w), g_{n} \in \mathbb{R}\right\}, \text { with } \quad e_{n}(w)=\operatorname{Im}\left(w^{n}\right) .
$$

As discussed below, the $m$-fold symmetric vortex patch solutions are essentially arising from bifurcations in the more restrictive function spaces

$$
X_{m}=\left\{f \in X, \text { s.t. } \forall w \in \mathbb{T}, f(w)=\sum_{n=1}^{+\infty} f_{n m-1} \bar{w}^{n m-1}\right\}
$$

and

$$
Y_{m}=\left\{g \in Y, \text { s.t. } \forall w \in \mathbb{T}, g(w)=\sum_{n=1}^{+\infty} g_{n} e_{n m}(w)\right\} .
$$

Of course, the spaces $X$ and $X_{m}$ are equipped with the strong topology of $C^{1+\alpha}$ whereas $Y$ and $Y_{m}$ are equipped with the strong topology of $C^{\alpha}$.

Next we recall the following lemma (see e.g. $[12,14]$ ).

Lemma 5.6. Let $\Delta=\{(w, w), w \in \mathbb{T}\}$ and let $K: \mathbb{T} \times \mathbb{T} \backslash \Delta \mapsto \mathbb{C}$ be a measurable function with the following properties. There exists $C>0$ such that,

$$
|K(w, \tau)| \leq C, \quad \forall(w, \tau) \notin \Delta
$$

and that for each $\tau \in \mathbb{T}$, the function $w \in \mathbb{T} \backslash\{\tau\} \mapsto K(w, \tau)$ is differentiable and

$$
\left|\partial_{w} K(w, \tau)\right| \leq \frac{C}{|w-\tau|} .
$$

Then the operator

$$
T \varphi(w)=\int_{\mathbb{T}} K(w, \tau) \varphi(\tau) d \tau
$$

sends $C^{\alpha}(\mathbb{T})$ to $L^{\infty}(\mathbb{T})$ for any $\alpha \in(0,1)$ with

$$
\|T \varphi\|_{\alpha} \leq C_{\alpha} C\|\varphi\|_{\infty}, \varphi \in L^{\infty}(\mathbb{T})
$$

where $C_{\alpha}$ depends only on $\alpha$.

5.4. Regularity of the functional I. The main goal of this section is to study the regularity properties required by Theorem 5.4 and Theorem 5.5 for the functional $G$ introduced in (4.6). Denote by $B_{r}$ the ball of center Id and radius $r$ in the space $X$ and $B_{r}^{m}$ the same ball in the space $X_{m}$.

Proposition 5.7. There exists $r \in(0,1)$ such that for any $\alpha \in(0,1)$ the following holds true.

(1) $G: \mathbb{R} \times \mathbb{R} \times B_{r} \rightarrow Y$ is of class $C^{1}$. It is at least of class $C^{3}$.

(2) The restriction $G: \mathbb{R} \times \mathbb{R} \times B_{r}^{m} \rightarrow Y_{m}$ is well-defined. 
Proof. The proofs are classical and can be performed in a similar way to those of [12, 14], using Lemma 5.6 in particular. Some details will be given later in the subsection 6.3 . Thus we only sketch the proof of the symmetry given in point (2). The spaces that used are described in subsection 5.3. Recall that

$$
B_{r}^{m}=\left\{\Phi \in X_{m},\|\Phi-\mathrm{Id}\|_{C^{1+\alpha}(\mathbb{T})} \leq r\right\}, \quad \Phi(w)=w+\sum_{n=1}^{+\infty} f_{n m-1} \bar{w}^{n m-1}
$$

and

$$
G(\varepsilon, \Omega, \Phi)=\operatorname{Im}\left\{(\Omega \Phi(w)-I(\varepsilon, \Phi)(w)) \overline{\Phi^{\prime}(w)} \bar{w}\right\}
$$

with

$$
I(\varepsilon, \Phi)(w)=f_{\mathbb{T}} \Phi^{\prime}(\tau) K_{0}(|\varepsilon||\Phi(w)-\Phi(\tau)|) d \tau .
$$

We begin by checking that $G(\varepsilon, \Omega, f)$ belongs to $Y_{m}$. It is enough for that purpose to prove that

$$
G(\varepsilon, \Omega, \Phi)\left(e^{\frac{2 i \pi}{m}} w\right)=G(\varepsilon, \Omega, \Phi)(w), \forall w \in \mathbb{T} .
$$

Note that

$$
\Phi\left(e^{\frac{2 i \pi}{m}} w\right)=e^{\frac{2 i \pi}{m}} \Phi(w), \quad \Phi^{\prime}\left(e^{\frac{2 i \pi}{m}} w\right)=\Phi^{\prime}(w)
$$

and thus the property is obvious for the first term $\operatorname{Im}\left\{\Omega \Phi(w) \overline{\Phi^{\prime}(w)} \bar{w}\right\}$. For the last term of $G$, it is enough to check the identity,

$$
I(\varepsilon, \Phi)\left(e^{\frac{2 i \pi}{m}} w\right)=e^{\frac{2 i \pi}{m}} I(\varepsilon, \Phi)(w), \forall w \in \mathbb{T} .
$$

This follows simply by making the change of variable $\tau=e^{\frac{2 i \pi}{m}} \xi$ :

$$
\begin{aligned}
I(\varepsilon, \Phi)\left(e^{\frac{2 i \pi}{m}} w\right) & =f_{\mathbb{T}} e^{\frac{2 i \pi}{m}} \Phi^{\prime}\left(e^{\frac{2 i \pi}{m}} \tau\right) K_{0}\left(|\varepsilon|\left|\Phi\left(e^{\frac{2 i \pi}{m}} w\right)-\Phi\left(e^{\frac{2 i \pi}{m}} \tau\right)\right|\right) d \tau \\
& =e^{\frac{2 i \pi}{m}} f_{\mathbb{T}} \Phi^{\prime}(\tau) K_{0}(|\varepsilon||\Phi(w)-\Phi(\tau)|) d \tau \\
& =e^{\frac{2 i \pi}{m}} I(\varepsilon, \Phi)(w) .
\end{aligned}
$$

This ends the proof.

5.5. Spectral study. In this section we compute the linearized operator at the trivial solution of the functional $G$ introduced in (4.6). We prove that it acts as a Fourier multiplier with symbol related to modified Bessel functions. This allows us to describe the full range of $\Omega$ corresponding to non-trivial kernels. Finally, we check that for these values of $\Omega$ all the assumptions of CrandallRabinowitz's theorem are satisfied.

5.5.1. Structure of the linearized operator. We prove the following result.

Proposition 5.8. Let $h: w \mapsto \sum_{n=0}^{+\infty} a_{n} \bar{w}^{n} \in X$, then

$$
D_{f} G(\varepsilon, \Omega, \operatorname{Id})(h)(w)=\sum_{n=0}^{+\infty} a_{n}(n+1)\left(\Omega_{n+1}(\varepsilon)-\Omega\right) e_{n+1}(w), \text { with } e_{n}(w)=\operatorname{Im}\left(w^{n}\right)
$$


and

$$
\Omega_{m}(\varepsilon)=I_{1}(|\varepsilon|) K_{1}(|\varepsilon|)-I_{m}(|\varepsilon|) K_{m}(|\varepsilon|)
$$

Proof. Without loss of generality, we may assume that $\varepsilon>0$. Now, for given $h \in X$, one may deduce from straightforward computations that

$$
D_{f} G(\varepsilon, \Omega, \operatorname{Id})(h)(w)=\mathcal{L}_{0}(h)(w)+\mathcal{L}_{1}(h)(w)+\mathcal{L}_{2}(h)(w)
$$

with

$$
\begin{gathered}
\mathcal{L}_{0}(h)(w)=\Omega \operatorname{Im}\left\{h(w) \bar{w}+\overline{h^{\prime}(w)}\right\}, \\
\mathcal{L}_{1}(h)(w)=\operatorname{Im}\left\{-\overline{h^{\prime}(w)} \bar{w} f_{\mathbb{T}} K_{0}(\varepsilon|w-\tau|) d \tau-\bar{w} f_{\mathbb{T}} h^{\prime}(\tau) K_{0}(\varepsilon|w-\tau|) d \tau\right\}
\end{gathered}
$$

and

$$
\mathcal{L}_{2}(h)(w)=\varepsilon \operatorname{Im}\left\{-\bar{w} f_{\mathbb{T}} \frac{\operatorname{Re}((h(w)-h(\tau))(\bar{w}-\bar{\tau}))}{|w-\tau|} K_{0}^{\prime}(\varepsilon|w-\tau|) d \tau\right\} .
$$

We begin with the easier term $\mathcal{L}_{0}(h)(w)$ whose computation is straightforward:

$$
\mathcal{L}_{0}(h)(w)=-\sum_{n=0}^{+\infty} a_{n} \Omega(n+1) e_{n+1} .
$$

For $\mathcal{L}_{1}(h)$ we first use the change of variable $\tau \mapsto w \tau$

$$
\mathcal{L}_{1}(h)(w)=\operatorname{Im}\left\{-\overline{h^{\prime}(w)} f_{\mathbb{T}} K_{0}(\varepsilon|1-\tau|) d \tau-f_{\mathbb{T}} h^{\prime}(\tau w) K_{0}(\varepsilon|1-\tau|) d \tau\right\}
$$

which implies that

$$
\mathcal{L}_{1}(h)(w)=-\sum_{n=1}^{+\infty} n a_{n}\left[f_{\mathbb{T}} K_{0}(\varepsilon|1-\tau|)\left(\bar{\tau}^{n+1}-1\right) d \tau\right] e_{n+1} .
$$

We focus on the integral term involving in $\mathcal{L}_{1}(h)(w)$. By symmetry arguments we obtain

$$
\begin{aligned}
f_{\mathbb{T}} K_{0}(\varepsilon|1-\tau|)\left(\bar{\tau}^{n+1}-1\right) d \tau & =\frac{1}{2 \pi} \int_{0}^{2 \pi} K_{0}(2 \varepsilon \sin (\theta / 2))(\cos (n \theta)-\cos \theta) d \theta \\
& =\frac{2}{\pi} \int_{0}^{\frac{\pi}{2}} K_{0}(2 \varepsilon \sin \theta)(\cos (2 n \theta)-\cos (2 \theta)) d \theta \\
& =\frac{2}{\pi} \int_{0}^{\frac{\pi}{2}} K_{0}(2 \varepsilon \cos \theta)\left((-1)^{n} \cos (2 n \theta)+\cos (2 \theta)\right) d \theta
\end{aligned}
$$

Using (4.4) we deduce that

$$
f_{\mathbb{T}} K_{0}(\varepsilon|1-\tau|)\left(\bar{\tau}^{n+1}-1\right) d \tau=I_{n}(\varepsilon) K_{n}(\varepsilon)-I_{1}(\varepsilon) K_{1}(\varepsilon) .
$$

Therefore

$$
\mathcal{L}_{1}(h)(w)=\sum_{n=1}^{+\infty} n a_{n}\left(I_{1}(\varepsilon) K_{1}(\varepsilon)-I_{n}(\varepsilon) K_{n}(\varepsilon)\right) e_{n+1} .
$$

For the computation of $\mathcal{L}_{2}(h)(w)$, we write

$$
\mathcal{L}_{2}(h)(w)=-\sum_{n=1}^{+\infty} \frac{\varepsilon a_{n}}{2}\left(f_{\mathbb{T}}\left[\frac{\left(\tau^{n}-1\right)(\tau-1)}{|1-\tau|}-\frac{\left(\bar{\tau}^{n}-1\right)(\bar{\tau}-1)}{|1-\tau|}\right] K_{0}^{\prime}(\varepsilon|1-\tau|) d \tau\right) e_{n+1} .
$$


Now we compute the following integral term which is more delicate

$$
d_{n} \triangleq \frac{\varepsilon}{2} f_{\mathbb{T}}\left[\frac{\left(\tau^{n}-1\right)(\tau-1)}{|1-\tau|}-\frac{\left(\bar{\tau}^{n}-1\right)(\bar{\tau}-1)}{|1-\tau|}\right] K_{0}^{\prime}(\varepsilon|1-\tau|) d \tau .
$$

First we use the following trigonometric identity: for $\tau=e^{i \theta}, \theta \in[0,2 \pi]$, one has

$$
\operatorname{Re}\left\{\left(\frac{\left(\tau^{n}-1\right)(\tau-1)}{|1-\tau|}-\frac{\left(\bar{\tau}^{n}-1\right)(\bar{\tau}-1)}{|1-\tau|}\right) \frac{d \tau}{2 i \pi}\right\}=\frac{1}{\pi} \cos (\theta / 2)(\sin \theta+\sin (n \theta)-\sin ((n+1) \theta)) d \theta .
$$

Thus integration by parts yields

$$
\begin{aligned}
d_{n} & =\frac{1}{2 \pi} \int_{0}^{2 \pi}(\varepsilon \cos (\theta / 2)) K_{0}^{\prime}(2 \varepsilon \sin (\theta / 2))(\sin \theta+\sin (n \theta)-\sin ((n+1) \theta)) d \theta \\
& =-\frac{1}{2 \pi} \int_{0}^{2 \pi} K_{0}(2 \varepsilon \sin (\theta / 2))(\cos \theta+n \cos (n \theta)-(n+1) \cos ((n+1) \theta)) d \theta .
\end{aligned}
$$

Performing a change of variables and invoking symmetry arguments imply

$$
\begin{aligned}
d_{n} & =-\frac{2}{\pi} \int_{0}^{\frac{\pi}{2}} K_{0}(2 \varepsilon \sin (\theta))(\cos (2 \theta)+n \cos (2 n \theta)-(n+1) \cos (2(n+1) \theta)) d \theta \\
& =\frac{2}{\pi} \int_{0}^{\frac{\pi}{2}} K_{0}(2 \varepsilon \cos (\theta))\left(\cos (2 \theta)-n(-1)^{n} \cos (2 n \theta)-(n+1)(-1)^{n} \cos (2(n+1) \theta)\right) d \theta .
\end{aligned}
$$

Using (4.4) we obtain

$$
d_{n}=-I_{1}(\varepsilon) K_{1}(\varepsilon)-n I_{n}(\varepsilon) K_{n}(\varepsilon)+(n+1) I_{n+1}(\varepsilon) K_{n+1}(\varepsilon) .
$$

Combined with (5.8) we find that

$$
\mathcal{L}_{1}(h)(w)+\mathcal{L}_{2}(h)(w)=\sum_{n=0}^{+\infty}(n+1) a_{n}\left(I_{1}(\varepsilon) K_{1}(\varepsilon)-I_{n+1}(\varepsilon) K_{n+1}(\varepsilon)\right) e_{n+1} .
$$

Putting together this identity with (5.7) gives the desired result.

5.5.2. Bifurcation assumptions. Next we check the assumptions on the linearized operator required by Theorem 5.4 and Theorem 5.5. For this purpose, we introduce the countable dispersion set

$$
\mathbb{S}=\left\{\Omega_{m}(\varepsilon) \triangleq I_{1}(|\varepsilon|) K_{1}(|\varepsilon|)-I_{m}(|\varepsilon|) K_{m}(|\varepsilon|), \quad m \geq 1\right\} .
$$

The main result reads as follows.

Proposition 5.9. Let $\varepsilon \in \mathbb{R}$ be a fixed real number and $G$ be the functional defined in (4.6); note that some of its properties are detailed in Proposition 5.7. Then the following assertions hold.

(1) The sequence $m \mapsto \Omega_{m}(\varepsilon)$ is strictly increasing and converges to $I_{1}(|\varepsilon|) K_{1}(|\varepsilon|)$.

(2) The kernel of $D_{f} G(\varepsilon, \Omega, \mathrm{Id})$ is non-trivial if and only if $\Omega=\Omega_{m}(\varepsilon) \in \mathbb{S}$. In this case, it is one-dimensional and generated by

$$
v_{m}: w \in \mathbb{T} \mapsto \bar{w}^{m-1} .
$$

(3) The range of $D_{f} G\left(\varepsilon, \Omega_{m}(\varepsilon), \mathrm{Id}\right)$ is closed in $Y$ and is of co-dimension one. It is given by

$$
\mathcal{R}\left(D_{f} G\left(\varepsilon, \Omega_{m}(\varepsilon), \mathrm{Id}\right)\right)=\left\{g \in C^{\alpha}(\mathbb{T}), g=\sum_{\substack{n \neq m \\ n=1}}^{+\infty} g_{n} e_{n}, g_{n} \in \mathbb{R}\right\} .
$$


(4) Transversality assumption:

$$
\partial_{\Omega} D_{f} G\left(\varepsilon, \Omega_{m}, \text { Id }\right) v_{m} \notin \mathcal{R}\left(D_{f} G\left(\varepsilon, \Omega_{m}, I d\right)\right) .
$$

Proof. (1) We use the following inequality (see [26]). For $\nu \geq 0$ and $x>0$

$$
\frac{I_{\nu+\frac{1}{2}}(x)}{I_{\nu-\frac{1}{2}}(x)}<\frac{x}{\nu+\sqrt{\nu^{2}+x^{2}}} \leq \frac{K_{\nu-\frac{1}{2}}(x)}{K_{\nu+\frac{1}{2}}(x)} .
$$

Thus using the positivity of $I_{n}$ and $K_{n}$ mentioned in (4.2), we find that the sequence $n \mapsto$ $I_{n}(|\varepsilon|) K_{n}(|\varepsilon|)$ is strictly decreasing. It remains to check that $\lim _{n \rightarrow \infty} I_{n}(|\varepsilon|) K_{n}(|\varepsilon|)=0$. For this, we establish a precise result on the convergence rate used below: there exists $C>0$ such that for any real number $\varepsilon$,

$$
\forall n \in \mathbb{N}^{\star}, \quad 0<I_{n}(|\varepsilon|) K_{n}(|\varepsilon|) \leq C \frac{\ln (n+1)}{n} .
$$

Indeed, using integration by parts in (4.4) we find

$$
I_{n}(|\varepsilon|) K_{n}(|\varepsilon|)=-\frac{2(-1)^{n} \varepsilon}{\pi n} \int_{0}^{\frac{\pi}{2}} \sin \theta K_{1}(2|\varepsilon| \cos \theta) \sin (2 n \theta) d \theta
$$

Thus

$$
0<I_{n}(|\varepsilon|) K_{n}(|\varepsilon|) \leq \frac{2|\varepsilon|}{\pi n} \int_{0}^{\frac{\pi}{2}} \sin \theta K_{1}(2|\varepsilon| \cos \theta)|\sin (2 n \theta)| d \theta .
$$

On the other hand using (4.3) we deduce by the change of variable $\theta=\cosh t$ that for $x>0$

$$
\begin{aligned}
K_{1}(x) & =\int_{1}^{+\infty} e^{-x \theta} \frac{\theta}{\sqrt{\theta^{2}+1}} d \theta \\
& =\int_{1}^{2} e^{-x \theta} \frac{\theta}{\sqrt{\theta^{2}+1}} d \theta+\int_{2}^{+\infty} e^{-x \theta} \frac{\theta}{\sqrt{\theta^{2}+1}} d \theta \\
& \leq e^{-x}+\int_{2}^{+\infty} e^{-x \theta} d \theta \\
& \leq e^{-x}+\frac{1}{x} e^{-2 x} .
\end{aligned}
$$

Consequently there exists $C>0$ such that for any $x>0$,

$$
K_{1}(x) \leq \frac{C}{x}
$$

which implies after straightforward computations related to Dirichlet kernel,

$$
\begin{aligned}
\forall n \in \mathbb{N}^{\star}, \quad I_{n}(\varepsilon) K_{n}(\varepsilon) & \leq \frac{C}{n} \int_{0}^{\frac{\pi}{2}} \frac{|\sin (2 n \theta)|}{\cos \theta} d \theta \\
& \leq \frac{C}{n} \int_{0}^{\frac{\pi}{2}} \frac{|\sin (2 n \theta)|}{\sin \theta} d \theta \\
& \leq C \frac{\ln (n+1)}{n} .
\end{aligned}
$$

(2) The result follows from the structure of the linearized operator stated in Proposition 5.8 and the strict monotonicity of the eigenvalues $\left(\Omega_{m}(\varepsilon)\right)_{m \geq 1}$.

(3) We want to prove that for any $m \geq 1$ the range of $D_{f} G\left(\varepsilon, \Omega_{m}(\varepsilon)\right.$, Id) coincides with

$$
Z_{m} \triangleq\left\{g \in C^{\alpha}(\mathbb{T}), g(w)=\sum_{\substack{n \neq m \\ n=1}}^{+\infty} g_{n} e_{n}, g_{n} \in \mathbb{R}\right\} .
$$


As $Z_{m}$ is closed in $\mathrm{Y}$ and of co-dimension one, it is enough to check that the range is $Z_{m}$. First, it is obvious that

$$
\mathcal{R}\left(D_{f} G\left(\varepsilon, \Omega_{m}(\varepsilon), \mathrm{Id}\right) \subset Z_{m}\right.
$$

and it thus remains to check the reverse inclusion. Let $g=\sum_{n \geq 1} g_{n} e_{n} \in Z_{m}$; we want to find $h \in X$ such that

$$
D_{f} G\left(\varepsilon, \Omega_{m}(\varepsilon), \operatorname{Id}\right)(h)=g .
$$

Set $h(w)=\sum_{n \geq 0} h_{n} \bar{w}^{n}$, then the equation

$$
D_{f} G\left(\varepsilon, \Omega_{m}(\varepsilon), \mathrm{Id}\right) h=g
$$

admits an explicit solution such that

$$
h_{n}=\frac{g_{n+1}}{(n+1)\left(\Omega_{n+1}(\varepsilon)-\Omega_{m}(\varepsilon)\right)}, \quad n \neq m-1
$$

and

$$
h_{m-1}=0 .
$$

We next check that $h \in C^{1+\alpha}(\mathbb{T})$. Since

$$
h(w)=\sum_{\substack{n \neq m-1 \\ n \geq 0}} \frac{g_{n+1}}{(n+1)\left(\Omega_{n+1}(\varepsilon)-\Omega_{m}(\varepsilon)\right)} \bar{w}^{n}
$$

then it follows from Cauchy-Schwarz inequality and the Bessel identity that

$$
\begin{aligned}
\|h\|_{L^{\infty}(\mathbb{T})} & \leq C_{0} \sum_{n \geq 1} \frac{\left|g_{n+1}\right|}{n+1} \\
& \leq C\|g\|_{L^{2}(\mathbb{T})} \\
& \leq C\|g\|_{C^{\alpha}(\mathbb{T})}
\end{aligned}
$$

where $C_{0}$ is the inverse of the distance between $\Omega_{m}(\varepsilon)$ and $\mathbb{S} \backslash\left\{\Omega_{m}(\varepsilon)\right\} . C_{0}$ is finite due to the monotonicity of the eigenvalues. We now prove that the derivative $h^{\prime}$ belongs to $C^{\alpha}$. It is obvious that

$$
h^{\prime}(w)=\sum_{\substack{n \neq m-1 \\ n=1}}^{+\infty} \frac{n g_{n+1}}{(n+1)\left(\Omega_{m}(\varepsilon)-\Omega_{n+1}(\varepsilon)\right)} \bar{w}^{n+1},
$$

which can be split as follows

$$
\begin{aligned}
h^{\prime}(w) & =\sum_{\substack{n \neq m \\
n=2}}^{+\infty} \frac{g_{n}}{\Omega_{m}(\varepsilon)-\Omega_{n}(\varepsilon)} \bar{w}^{n}+\sum_{\substack{n \neq m \\
n=2}}^{+\infty} \frac{g_{n}}{n\left(\Omega_{n}(\varepsilon)-\Omega_{m}(\varepsilon)\right)} \bar{w}^{n} \\
= & -\sum_{\substack{n \neq m \\
n=2}}^{+\infty} \frac{g_{n}}{K_{m}(|\varepsilon|) I_{m}(|\varepsilon|)} \bar{w}^{n}-\sum_{\substack{n \neq m \\
n=2}}^{+\infty} g_{n}\left[\frac{1}{\Omega_{n}(\varepsilon)-\Omega_{m}(\varepsilon)}-\frac{1}{K_{m}(|\varepsilon|) I_{m}(|\varepsilon|)}\right] \bar{w}^{n} \\
& +\sum_{\substack{n \neq m \\
n=2}}^{+\infty} \frac{g_{n}}{n K_{m}(|\varepsilon|) I_{m}(|\varepsilon|)} \bar{w}^{n}+\sum_{\substack{n \neq m \\
n=2}}^{+\infty} \frac{g_{n}}{n}\left[\frac{1}{\Omega_{n}(\varepsilon)-\Omega_{m}(\varepsilon)}-\frac{1}{K_{m}(|\varepsilon|) I_{m}(|\varepsilon|)}\right] \bar{w}^{n} .
\end{aligned}
$$


Set

$$
\chi(w)=\sum_{\substack{n \neq m \\ n=2}}^{+\infty} g_{n} \bar{w}^{n}, \quad H_{1}(w)=\sum_{\substack{n \neq m \\ n=2}}^{+\infty}\left[\frac{1}{\Omega_{n}(\varepsilon)-\Omega_{m}(\varepsilon)}-\frac{1}{K_{m}(|\varepsilon|) I_{m}(|\varepsilon|)}\right] \bar{w}^{n}, \quad H_{2}(w)=\sum_{\substack{n \neq m \\ n=2}}^{+\infty} \frac{\bar{w}^{n}}{n}
$$

and

$$
H_{3}(w)=\sum_{\substack{n \neq m \\ n=2}}^{+\infty} \frac{1}{n}\left[\frac{1}{\Omega_{n}(\varepsilon)-\Omega_{m}(\varepsilon)}-\frac{1}{K_{m}(|\varepsilon|) I_{m}(|\varepsilon|)}\right] \bar{w}^{n}
$$

Then

$$
h^{\prime}(w)=-\frac{1}{K_{m}(|\varepsilon|) I_{m}(|\varepsilon|)} \chi(w)-\chi * H_{1}(w)+\frac{1}{K_{m}(|\varepsilon|) I_{m}(|\varepsilon|)} \chi * H_{2}(w)+\chi * H_{3}(w) .
$$

As $\chi(w)=\Pi_{+}(2 i g(w))-2 i g(w)$, with $\Pi_{+}$being the Szegö projection that sends continuously $C^{\alpha}(\mathbb{T})$ to itself, we deduce that $\chi \in C^{\alpha}(\mathbb{T})$. Recall that Szegö projection is defined by

$$
w \in \mathbb{T}, \quad f(w)=\sum_{n \in \mathbb{Z}} a_{n} w^{n}, \quad \Pi_{+} f(w)=\sum_{n \in \mathbb{N}} a_{n} w^{n} .
$$

Hence in order to ensure $h^{\prime} \in C^{\alpha}(\mathbb{T})$ it is enough to prove that $H_{j} \in L^{1}(\mathbb{T}), j \in\{1,2,3\}$. Let us start with $H_{1}$. It is obvious that

$$
\left|\frac{1}{\Omega_{n}(\varepsilon)-\Omega_{m}(\varepsilon)}-\frac{1}{K_{m}(|\varepsilon|) I_{m}(|\varepsilon|)}\right|=\left|\frac{K_{n}(|\varepsilon|) I_{n}(|\varepsilon|)}{K_{m}(|\varepsilon|) I_{m}(|\varepsilon|)\left(K_{m}(|\varepsilon|) I_{m}(l \varepsilon \mid)-K_{n}(|\varepsilon|) I_{n}(|\varepsilon|)\right)}\right| .
$$

Hence using (5.12) we find a constant $C$ depending on $m$ and $\varepsilon$ such that for any $n \neq m$

$$
\begin{aligned}
\left|\frac{1}{\Omega_{n}(\varepsilon)-\Omega_{m}(\varepsilon)}-\frac{1}{K_{m}(|\varepsilon|) I_{m}(|\varepsilon|)}\right| & \leq C K_{n}(|\varepsilon|) I_{n}(|\varepsilon|) \\
& \leq C \frac{\ln (n+1)}{n} .
\end{aligned}
$$

According to the Parseval identity, this proves that $H_{1} \in L^{2}(\mathbb{T})$ and by the usual embedding we find $H_{1} \in L^{1}(\mathbb{T})$. It is simple to check that $H_{2}, H_{3}$ belong to $L^{2}(\mathbb{T})$ and so to $L^{1}(\mathbb{T})$ which completes the desired result.

(4) For the transversality assumption, it is obvious that for any $h \in X$

$$
\partial_{\Omega} D_{f} G\left(\varepsilon, \Omega_{m}(\varepsilon), \operatorname{Id}\right) h=\operatorname{Im}\left\{h(w) \bar{w}+\overline{h^{\prime}(w)}\right\} .
$$

Therefore, for $v_{m}(w)=\bar{w}^{m-1}$

$$
\partial_{\Omega} D_{f} G\left(\varepsilon, \Omega_{m}(\varepsilon), \mathrm{Id}\right) v_{m}=-m e_{m} \notin \mathcal{R}\left(D_{f} G\left(\varepsilon, \Omega_{m}(\varepsilon), \mathrm{Id}\right)\right)
$$

and consequently the transversality condition is verified.

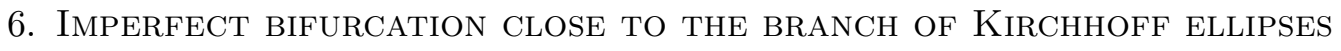

This section is devoted to the study of the global structure of the two-fold branch. According to Theorem 5.1 we know there exists a local branch close to Rankine vortices that bifurcates at the point $\Omega_{2}(\varepsilon)$. For $\varepsilon=0$ the full branch is explicitly described by Kirchhoff ellipses, and according to $[3,15,16,20]$ we know that from this branch a countable family of bifurcating curves emerges at the Love instability points [19]. Notice that these new curves model alternating one/two-fold $\mathrm{V}$-states and the two-fold $\mathrm{V}$-states are characterized by an odd frequency perturbation of the conformal mapping of the ellipse $w \in \mathbb{T} \mapsto w+Q \bar{w}$. 
We investigate below the 'imperfect' bifurcation, that is, the behavior of the solution branch structure subject to a small perturbation in $\varepsilon$. We prove that the scenarios of persistence/breakdown symmetry occur simultaneously close to the Kirchhoff ellipse branch. Indeed, we prove by using perturbation theory, see Theorem 6.3, that far from the second bifurcating point the local structure of the two-fold branch persists and varies continuously with respect to a small perturbation in $\varepsilon$. However around the singularity set the issue depends on the symmetry of the V-states. In fact, we show in Theorem 6.3 that the diagram structure around the one-fold bifurcating curves is not destroyed and is similar to the Euler one. However, and this is only proved for the $m=4$ Love instability point, the symmetry is broken down around the first bifurcating curve of the two-fold $\mathrm{V}$-states (see Theorem 6.5). This is a kind of resonance phenomenon between the two branches with the same symmetry leading to a separation of the singularity and a loss of the connectedness. Numerically, in Section 3 this behavior is observed for the first two-fold branches emerging from the ellipse, but from an analytical standpoint the problem is difficult due to the cumbersome computations required for higher elliptical azimuthal wavenumbers.

6.1. Function spaces II. We first introduce the function spaces suitable for studying the bifurcation from the two-fold branch. We draw attention to the fact that we use the same notation as in the Section 5 dealing with the $m$-folds structure but with a different meaning. For $\alpha \in(0,1)$, we set

$$
X=\left\{f \in C^{1+\alpha}(\mathbb{T}), f(w)=\sum_{n=2}^{+\infty} f_{n} w^{n}, f_{n} \in \mathbb{R}\right\}
$$

and

$$
Y=\left\{g \in C^{\alpha}(\mathbb{T}), g(w)=\sum_{n=1}^{+\infty} g_{n} e_{n}(w), g_{n} \in \mathbb{R}\right\}, \text { with } \quad e_{n}(w)=\operatorname{Im}\left(w^{n}\right) .
$$

6.2. Summary of the bifurcations from Kirchhoff ellipses. The results of this section were obtained in [15] and for the commodity of the presentation we briefly recall them. Since ellipses are explicit rotating solutions for the Euler equations, then from [15] one finds that

$$
G\left(0, \frac{1-Q^{2}}{4}, \alpha_{Q}\right)=0, \quad \forall Q \in(0,1)
$$

with $\alpha_{Q}: w \in \mathbb{T} \mapsto w+Q \bar{w}$ being the conformal parametrization of the ellipse centered at the origin and with semi-axes $1 \pm Q$ and $Q \in[0,1)$. Notice that Kirchhoff discovered that such ellipses rotate at the angular velocity $\frac{1-Q^{2}}{4}$. Introducing

$$
F(\varepsilon, Q, f)=G\left(\varepsilon, \frac{1-Q^{2}}{4}, \alpha_{Q}+f\right), \quad f \in X
$$

where the space $X$ is described in (6.1), it is plain that

$$
F(0, Q, 0)=0, \quad \forall Q \in[0,1) .
$$

From (4.7) one obtains

$$
\mathcal{L}_{Q} \triangleq D_{f} F(0, Q, 0)=-D_{f} G_{E}\left(\frac{1-Q^{2}}{2}, \alpha_{Q}\right) .
$$

Let $m \geq 3$ be an integer and denote by $Q_{m}$ the unique solution in $[0,1)$ of the equation

$$
1+Q^{m}-\frac{1-Q^{2}}{2} m=0,
$$

and set

$$
\mathcal{S} \triangleq\left\{Q_{m}, m \geq 3\right\}
$$

At various points in the argument, we need to distinguish between the following two subsets of $\mathcal{S}$ :

$$
\mathcal{S}_{\text {reso }} \triangleq\left\{Q_{2 m}, m \geq 2\right\} \quad \underset{28}{\text { and }} \mathcal{S}_{\text {Nreso }} \triangleq\left\{Q_{2 m+1}, m \geq 1\right\} \text {. }
$$


The first one is called the 'resonant set' and the second is the 'non-resonant set'. Note that from [15] we know that the sequence $\left(Q_{m}\right)_{m \geq 3}$ is strictly increasing with

$$
\lim _{m \rightarrow+\infty} Q_{m}=1 \text {. }
$$

The following result dealing with the structure of the linearized operator $\mathcal{L}_{Q}$ was proved in [15]. This was used to prove the existence of bifurcations from Kirchhoff ellipses using the CrandallRabinowitz theorem.

Proposition 6.1. Let $X$ and $Y$ be the spaces introduced in (6.1) and (6.2). Then the following assertions hold true.

(1) Let $h(w)=\sum_{n \geq 2} a_{n} w^{n} \in X$, then

$$
\mathcal{L}_{Q} h=\frac{1}{2} \sum_{n \geq 1} g_{n+1} e_{n} ; \quad e_{n}(w)=\operatorname{Im}\left(w^{n}\right),
$$

with

$$
\begin{aligned}
g_{2} & =\frac{1}{2}(1+Q)^{2} a_{2}, \\
g_{3} & =2 Q^{2} a_{3}, \\
g_{n+1} & =\left(1+Q^{n}-\frac{1-Q^{2}}{2} n\right)\left(a_{n+1}-Q a_{n-1}\right), \quad \forall n \geq 3 .
\end{aligned}
$$

(2) The kernel of $\mathcal{L}_{Q}$ is non-trivial if and only if $Q=Q_{m} \in \mathcal{S}$ and it is a one-dimensional vector space generated by

$$
v_{m}(w)=\frac{w^{m+1}}{1-Q w^{2}}
$$

(3) The range of $\mathcal{L}_{Q}$ is of co-dimension one in $Y$ and it is given by

$$
R\left(\mathcal{L}_{Q}\right)=\left\{g \in C^{\alpha}(\mathbb{T}), g=\sum_{\substack{n \geq 1 \\ n \neq m}} g_{n+1} e_{n}, \quad g_{n} \in \mathbb{R}\right\} .
$$

(4) Transversality assumption: for any $Q=Q_{m} \in \mathcal{S}$,

$$
\partial_{Q} \mathcal{L}_{Q} v_{m} \notin R\left(\mathcal{L}_{Q}\right)
$$

6.3. Regularity of the functional II. The main goal of this section is to study the regularity properties required by Theorem 5.4 for the functional $F$ introduced in (6.3).

Proposition 6.2. Let $\alpha \in(0,1), \mu \in(0,1)$ and set $r_{\mu}=\frac{1-\mu}{2}$. Then we have

$$
\begin{array}{ll}
F:(-1,1) \times(0, \mu) \times B_{r_{\mu}} & \longrightarrow Y \\
(\varepsilon, Q, f) & \longmapsto F(\varepsilon, \Omega, f)
\end{array}
$$

is well-defined and of class $C^{1}$, and $\partial_{Q} \partial_{f} F$ exists and is continuous on $(-1,1) \times(0, \mu) \times B_{r_{\mu}}$, where $B_{r_{\mu}}=\left\{f \in X,\|f\|_{C^{1+\alpha}} \leq r_{\mu}\right\}$. Moreover for any $i, j \in \mathbb{N}, i+j \leq 3$ the function $\partial_{\Omega}^{i} \partial_{f}^{j} F(\varepsilon, .,$.$) is$ continuous.

Proof. We only sketch the basic steps of the proof which closely parallels the proof developed in [15]. For more details we refer the reader to this reference. First, we write

$$
\begin{aligned}
& F(\varepsilon, Q, f(w))=\operatorname{Im}\left\{\frac{1-Q^{2}}{4}\left(1+Q \bar{w}^{2}+\bar{w} f(w)\right)\left(1-Q w^{2}+\overline{f^{\prime}(w)}\right)\right. \\
& \left.-\overline{\Phi^{\prime}(w)} \bar{w} f_{\mathbb{T}} \Phi^{\prime}(\tau) K_{0}^{\varepsilon}(|\Phi(w)-\Phi(\tau)|) d \tau\right\}
\end{aligned}
$$


with the notation $\Phi(w)=\alpha_{q}(w)+f(w)$ and

$$
K_{0}^{\varepsilon}(x)=K_{0}(|\varepsilon| x)+\log \left(\frac{|\varepsilon|}{2}\right) .
$$

Since $C^{\alpha}$ is an algebra, $f \in C^{1+\alpha}$ and $f^{\prime} \in C^{\alpha}$, then the first function

$$
w \in \mathbb{T} \mapsto \frac{1-Q^{2}}{4} \operatorname{Im}\left\{\left[1+Q \bar{w}^{2}+\bar{w} f(w)\right]\left[1-Q w^{2}+\overline{f^{\prime}(w)}\right]\right\}
$$

belongs to $C^{\alpha}$ and its Fourier coefficients are all real; therefore, it belongs to the space $Y$. The $C^{1}$ regularity with respect to $(Q, f)$ is elementary and was discussed in [15]. For the second term, using the results in subsection 4.2 , one may write

$$
K_{0}^{\varepsilon}(x)=-\log (x)-\left(\log \left(\frac{|\varepsilon|}{2}\right)+\log (x)\right) \frac{\varepsilon^{2} x^{2}}{4} \mathcal{K}_{1}\left(\varepsilon^{2} x^{2}\right)+\mathcal{K}_{2}\left(\varepsilon^{2} x^{2}\right)
$$

where

$$
\mathcal{K}_{1}(z)=\sum_{m=1}^{+\infty} \frac{\left(\frac{z}{4}\right)^{m-1}}{(m !)^{2}}
$$

and

$$
\mathcal{K}_{2}(z)=\sum_{m=0}^{+\infty}\left(\frac{z}{4}\right)^{m} \frac{\psi(m+1)}{(m !)^{2}}
$$

Consequently,

$$
-f_{\mathbb{T}} \Phi^{\prime}(\tau) K_{0}^{\varepsilon}(|\Phi(w)-\Phi(\tau)|) d \tau=T_{0} \Phi^{\prime}(w)+T_{1} \Phi^{\prime}(w)+T_{2} \Phi^{\prime}(w)
$$

where

$$
\begin{gathered}
T_{0} \varphi(w)=f_{\mathbb{T}} \log (|\Phi(w)-\Phi(\tau)|) \varphi(\tau) d \tau, \\
T_{1} \varphi(w) \triangleq f_{\mathbb{T}} \widehat{K_{1}}(\tau, w, \varepsilon) \varphi(\tau) d \tau
\end{gathered}
$$

and

with

$$
T_{2} \varphi(w) \triangleq-f_{\mathbb{T}} \mathcal{K}_{2}\left(\varepsilon^{2}|\Phi(w)-\Phi(\tau)|^{2}\right) \varphi(\tau) d \tau
$$

$$
\widehat{K_{1}}(\tau, w, \varepsilon) \triangleq \frac{\varepsilon^{2}}{4}\left(\log \left(\frac{|\varepsilon|}{2}\right)+\log (|\Phi(w)-\Phi(\tau)|)\right)|\Phi(w)-\Phi(\tau)|^{2} \mathcal{K}_{1}\left(\varepsilon^{2}|\Phi(w)-\Phi(\tau)|^{2}\right)
$$

Moreover, we have seen in (4.8) that

$$
T_{0} \Phi^{\prime}(w)=\overline{\widehat{T}_{0} \Phi^{\prime}(w)}
$$

with

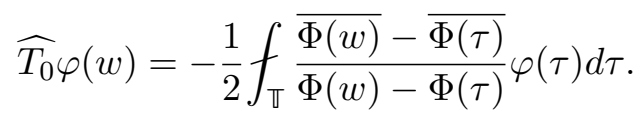

Let $Q \in(0, \mu)$ and take $r_{\mu}=\frac{1-\mu}{2}$. By the mean value theorem, there exists a constant $C_{\mu}$ such that for all $f \in B_{r_{\mu}}$

$$
\frac{1-\mu}{2}|\tau-w| \leq|\Phi(w)-\Phi(\tau)| \leq C_{\mu}|\tau-w|, \quad \forall \tau, w \in \mathbb{T} .
$$

In addition, we may easily check that the kernel $K(\tau, w)=\frac{\overline{\Phi(w)}-\overline{\Phi(\tau)}}{\Phi(w)-\Phi(\tau)}$ satisfies the assumptions of Lemma 5.6 and thus

$$
\left\|T_{0} \Phi^{\prime}\right\|_{C^{\alpha}(\mathbb{T})} \leq C\left\|\Phi^{\prime}\right\|_{L^{\infty}} \leq C_{0}
$$


Note that according to [15] we also have that $(\varepsilon, Q, f) \mapsto T_{0} \Phi^{\prime}$ is of class $C^{1}$ from $(-1,1) \times(0, \mu) \times B_{r_{\mu}}$ to $C^{\alpha}(\mathbb{T})$. As for $T_{2}$, the kernel is not singular and one may easily check that

$$
\left|\widehat{K}_{1}(\tau, w, \varepsilon)\right|+\left|\partial_{w} \widehat{K}_{1}(\tau, w, \varepsilon)\right| \leq C_{0}, \forall \tau, w \in \mathbb{T} .
$$

Consequently we may use once again Lemma 5.6 and deduce that $(\varepsilon, Q, f) \mapsto T_{1} \Phi^{\prime}$ is well-defined. Moreover the Fourier coefficients of $T_{1} \Phi^{\prime}$ are real which follows from the general fact

$$
\overline{T_{1} \varphi(w)}=T_{1} \varphi(\bar{w}), \forall \varphi \in X, \forall w \in \mathbb{T} .
$$

By straightforward arguments we can also prove that $(\varepsilon, Q, f) \mapsto T_{1} \Phi^{\prime}$ is of class $C^{1}$. Observe that the regularity with respect to $\varepsilon$ comes in particular from the fact that the function $\varepsilon \in(-1,1) \mapsto$ $\varepsilon^{2} \log \varepsilon$ is $C^{1}$. The same analysis can be implemented for the last term $T_{2} \Phi^{\prime}$ and this concludes the $C^{1}$ regularity of $(\varepsilon, Q, f) \mapsto F(\varepsilon, Q, f)$. Concerning the existence and the regularity of $\partial_{Q} \partial_{f} F$ it can be proved similarly to the case $\varepsilon=0$ discussed in [15].

6.4. Bifurcation diagram far from the resonant set. The main goal of this section is study the structure of the bifurcation diagram far from the resonant set $\mathcal{S}_{\text {reso }}$ defined in (6.6). We prove its persistence for small perturbations. This is done in two different subsections. First we prove the stability of the Kirchhoff ellipse branch under small perturbations, leading to the existence of a two-fold branch for (1.1) living close to the ellipse branch. Second we explore the bifurcation of one-fold curves from the two-fold branch close to the non-resonant set. This proves the persistence of the bifurcation diagram of the Euler equations under small perturbations in $\varepsilon$ but far from the resonant set.

6.4.1. Structure of the two-fold curve. The aim in this subsection is to construct two-fold V-states close to Kirchhoff ellipses $\mathcal{E}_{Q}$ parametrized by $w \in \mathbb{T} \mapsto w+Q \bar{w}$, with $Q \in[0,1)$. We first study the case where $Q$ is far from the resonant set $\mathcal{S}_{\text {reso }}=\left\{Q_{2 m}, m \in \mathbb{N}^{\star}\right\}$. We prove that a onedimensional continuous curve can be constructed away from this set and which remains close to Kirchhoff ellipses for small values of $\varepsilon$. For this purpose we introduce the spaces

$$
X_{2}=\left\{f \in C^{1+\alpha}(\mathbb{T}), f(w)=\sum_{n \in \mathbb{N}^{*}} f_{n} w^{2 n+1}, f_{n} \in \mathbb{R}\right\}
$$

and

$$
Y_{2}=\left\{g \in C^{\alpha}(\mathbb{T}), g(w)=\sum_{n \in \mathbb{N}^{*}} g_{n} e_{2 n}, g_{n} \in \mathbb{R}\right\}, \text { with } e_{n}(w)=\operatorname{Im}\left(w^{n}\right) .
$$

Note that a domain whose boundary is parametrized by $\Phi(w)=w+Q \bar{w}+f(w), w \in \mathbb{T}$ with $f \in X_{2}$ is two-fold. The main goal is to prove the following.

Theorem 6.3. Consider the V-state equation (6.3) and let $m \in \mathbb{N}^{*}, \delta<\frac{Q_{2 m+2}-Q_{2 m}}{2}$. Define $I_{m, \delta}=\left[Q_{2 m}+\delta, Q_{2 m+2}-\delta\right]$. Then there exists $\varepsilon_{0}>0$ and a function

$$
\begin{aligned}
f:\left[-\varepsilon_{0}, \varepsilon_{0}\right] \times I_{m, \delta} & \longrightarrow X_{2} \\
(\varepsilon, Q) & \longmapsto f(\varepsilon, Q) .
\end{aligned}
$$

of class $C^{1}$ such that

$$
F(\varepsilon, Q, f(\varepsilon, Q))=0, \quad \forall(\varepsilon, Q) \in\left[-\varepsilon_{0}, \varepsilon_{0}\right] \times I_{m, \delta} .
$$

In particular the curve $Q \in I_{m, \delta} \mapsto \alpha_{Q}+f(\varepsilon, Q)$ describes rotating patches with two-fold symmetry living close to Kirchhoff ellipses. 
Proof. The proof relies on the use of the implicit function theorem. First notice from Proposition 6.2 that for any $\mu \in(0,1)$, the functional

$$
\begin{aligned}
F:(-1,1) \times(0, \mu) \times B_{r_{\mu}}^{2} & \longrightarrow Y_{2} \\
(\varepsilon, Q, f) & \longmapsto F(\varepsilon, Q, f)
\end{aligned}
$$

is well-defined and of class $C^{1}$, where

$$
B_{r_{\mu}}^{2}=\left\{f \in X_{2},\|f\|_{C^{1+\alpha}} \leq r_{\mu}\right\} \quad \text { and } \quad r_{\mu}=\frac{1-\mu}{2} .
$$

We point out that the persistence of two-fold symmetry follows from Proposition 5.7. In addition, $D_{f} F(0, Q, 0)$ is given by the restriction of the operator $\mathcal{L}_{Q}$ described by (6.4) on the sub-space $X_{2}$. As we have seen in Proposition 6.1, the kernel of $\mathcal{L}_{Q}$ is non-trivial if and only if $Q \in \mathcal{S}$. Since $Q \in I_{m, \delta}$ then $\operatorname{Ker} D_{f} F(0, Q, 0)$ is trivial for any $Q \neq Q_{2 m+1}$, and for $Q=Q_{2 m+1}$ the kernel is one-dimensional and generated by the vector $v_{2 m+1}(w)=\frac{w^{2 m+2}}{1-Q w^{2}}$. However this vector does not belong to $X_{2}$ and consequently $\operatorname{Ker} D_{f} F\left(0, Q_{2 m+1}, 0\right)$ is also trivial. Therefore for any $Q \in I_{m, \delta}$ the linear operator $D_{f} F(0, Q, 0) \in \mathcal{L}\left(X_{2}, Y_{2}\right)$ is one-to-one. We check that it is also onto. Let $g=\sum_{n \geq 1} g_{n} e_{2 n} \in Y_{2}$ and consider finding the pre-image by $D_{f} F(0, Q, 0)$. Then according to Proposition 6.1, $h(w)=\sum_{n \geq 1} h_{n} w^{2 n+1}$ satisfies $D_{f} F(0, Q, 0) h=g$ if and only if

$$
g_{1}=2 Q^{2} h_{1} \quad \text { and } \quad g_{n}=\left(1+Q^{2 n}-\left(1-Q^{2}\right) n\right)\left(h_{n}-Q h_{n-1}\right), \forall n \geq 2 .
$$

Note that for each $n$ the number $\left(\left(1-Q^{2}\right) n-1-Q^{2 n}\right)$ vanishes if and only if $Q=Q_{2 n}$, and thus for $Q \in I_{m, \delta}$ this coefficient does not vanish uniformly in $n$. One can see from the recursion relation that

$$
h(w)=\frac{h_{1} w^{3}+G(w)}{1-Q w^{2}} \quad \text { with } \quad G(w)=\sum_{n \geq 2} \frac{g_{n}}{1+Q^{2 n}-\left(1-Q^{2}\right) n} w^{2 n+1} .
$$

Since $Q \in(0,1)$ and $\frac{1}{1-Q w^{2}}$ is $C^{\infty}(\mathbb{T})$, then $h \in X_{2}$ if and only if $G \in C^{1+\alpha}(\mathbb{T})$. Thus it suffices to establish $G^{\prime} \in C^{\alpha}(\mathbb{T})$ or equivalently

$$
\chi: w \in \mathbb{T} \mapsto \sum_{n \geq 2} \frac{n\left(1-Q^{2}\right) g_{n}}{1+Q^{2 n}-\left(1-Q^{2}\right) n} w^{2 n} \in C^{\alpha}(\mathbb{T}) .
$$

It is plain that

$$
\begin{aligned}
\chi(w) & =-\sum_{n \geq 2} g_{n} w^{2 n}+\sum_{n \geq 1} \frac{1+Q^{2 n}}{1+Q^{2 n}-\left(1-Q^{2}\right) n} g_{n} w^{2 n} \\
& =-\Pi^{+}\left(2 i g(w)-g_{1} w^{2}\right)+K \star \Pi^{+}\left(2 i g(w)-g_{1} w^{2}\right)
\end{aligned}
$$

with $\Pi^{+}$being the Szegö projection and

$$
K(w)=\sum_{n \geq 2} \frac{1+Q^{2 n}}{1+Q^{2 n}-\left(1-Q^{2}\right) n} w^{2 n}
$$

It is easy to prove the existence of a constant $C>0$ such that for any $Q \in I_{m, \delta}$

$$
\left|\frac{1+Q^{2 n}}{1+Q^{2 n}-\left(1-Q^{2}\right) n}\right| \leq \frac{C}{n} .
$$

Therefore $K \in L^{2}(\mathbb{T}) \subset L^{1}(\mathbb{T})$, combined with the fact $\Pi^{+} g \in C^{\alpha}(\mathbb{T})$, implies that $\chi \in C^{\alpha}(\mathbb{T})$. Finally we see that $D_{f} F(0, Q, 0)$ is onto and thus it is an isomorphism from $X_{2}$ to $Y_{2}$. By the 
implicit function theorem and a standard compactness argument we conclude the existence of a unique surface of solutions

$$
F(\varepsilon, Q, f(\varepsilon, Q))=0, \quad \forall(\varepsilon, Q) \in\left[-\varepsilon_{0}, \varepsilon_{0}\right] \times I_{m, \delta} .
$$

This achieves the proof of Theorem 6.3.

6.4.2. Bifurcation from the two-fold curve. In this subsection we prove the bifurcation of countable family of one-dimensional curves of $\mathrm{V}$-states from the curve constructed in Theorem 6.3 at some points which are close to the points of the non-resonant set $\mathcal{S}_{\text {Nreso }}=\left\{Q_{2 m+1}, m \geq 1\right\}$.

The main result may be stated as follows.

Theorem 6.4. Let $m \geq 3$ be an odd number. There exists $\varepsilon_{0}>0$ such that for any $\varepsilon \in\left[-\varepsilon_{0}, \varepsilon_{0}\right]$, there exists a one-dimensional curve of one-fold $V$-states bifurcating from the two-fold branch constructed in Theorem 6.3 at a point $Q_{\varepsilon, m}$ close to $Q_{m}$.

Proof. The proof follows the same lines of Theorem 5.5 with slight modifications using the Lyapunov-Schmidt reduction in an important way. First recall from Proposition 6.2 that the functional $F:(-1,1) \times(0, \mu) \times B_{r_{\mu}} \rightarrow Y$ is well-defined and is of class $C^{1}$, with

$$
B_{r_{\mu}}=\left\{f \in X,\|f\|_{C^{1+\alpha}} \leq r_{\mu}\right\} \quad \text { and } \quad r_{\mu}=\frac{1-\mu}{2} \text {. }
$$

According to Proposition 6.1, for $Q=Q_{m}$ the kernel of $\mathcal{L}_{Q_{m}}$ is generated by the vector $v_{m}(w)=$ $\frac{w^{m+1}}{1-Q w^{2}}$. Denote by $\mathcal{X}$ a complement of $v_{m}$ in $X$ such that

$$
X_{2} \subset \mathcal{X} .
$$

This last fact follows since $m$ is odd and therefore the function $v_{m}$ is even; consequently, we can choose a complement containing odd functions which is exactly the space $X_{2}$. Recall that the spaces $X$ and $X_{2}$ were introduced in (6.1) and (6.8). The range of $\mathcal{L}_{Q_{m}}$, denoted by $\mathcal{Y}$, is of co-dimension one and we may choose a complement generated by the vector $w_{m}=e_{m}$. Then

$$
X=\left\langle v_{m}\right\rangle \oplus \mathcal{X} \quad \text { and } \quad Y=\left\langle w_{m}\right\rangle \oplus \mathcal{Y} .
$$

Let $\Pi_{1}: X \mapsto\left\langle v_{m}\right\rangle$ be the projection along $\mathcal{X}$ onto $\left\langle v_{m}\right\rangle$. Thus

$$
f=s v_{m}+g, \quad g \in \mathcal{X} \Longrightarrow \Pi_{1} f=s v_{m},
$$

and similarly define the projection $\Pi_{2}: Y \mapsto\left\langle w_{m}\right\rangle$ along $\mathcal{Y}$ onto $\left\langle w_{m}\right\rangle$. The V-state equation $F(\varepsilon, Q, f)=0$ is then equivalent to the system

$$
F_{1}(\varepsilon, Q, s, g) \triangleq\left(\mathrm{Id}-\Pi_{2}\right) F\left(\varepsilon, Q, s v_{m}+g\right)=0
$$

and

$$
F_{2}(\varepsilon, Q, s, g) \triangleq \Pi_{2} F\left(\varepsilon, Q, s v_{m}+g\right)=0 .
$$

The function $F_{1}:(-1,1) \times(0, \mu) \times(-\eta, \eta) \times B_{r} \rightarrow \mathcal{Y}$ is $C^{1}$, with $B_{r}$ a small ball in $\mathcal{X}$ centered at 0 , and $\eta>0$ such that for any $s \in(-\eta, \eta)$ and for any $g \in B_{r}$ we have $s v_{m}+g \in B_{r_{\mu}}$. Moreover,

$$
F_{1}\left(0, Q_{m}, 0,0\right)=0
$$

and it is not difficult to check that

$$
\partial_{g} F_{1}\left(0, Q_{m}, 0,0\right)=\left(\mathrm{Id}-\Pi_{2}\right) \partial_{f} F\left(0, Q_{m}, 0\right): \mathcal{X} \rightarrow \mathcal{Y}
$$

is an isomorphism. By the implicit function theorem the solutions of the equation $F_{1}(\varepsilon, Q, s, g)=0$ are described near the point $\left(0, Q_{m}, 0,0\right)$ by the parametrization $g=\varphi(\varepsilon, Q, s)$ with

$$
\varphi:(-\delta, \delta) \times\left(Q_{m}-\delta, Q_{m}+\delta\right) \times(-\delta, \delta) \rightarrow \mathcal{X}, \quad \delta>0
$$


being a $C^{1}$ function. By virtue of Theorem 6.3, we know the existence of a function

$$
(\varepsilon, Q) \mapsto f(\varepsilon, Q) \in X_{2} \subset \mathcal{X}
$$

such that

$$
F(\varepsilon, Q, f(\varepsilon, Q))=0
$$

and so in particular

$$
F_{1}(\varepsilon, Q, 0, f(\varepsilon, Q))=0
$$

Thus by uniqueness we get

$$
\varphi(\varepsilon, Q, 0)=f(\varepsilon, Q), \quad \forall(\varepsilon, Q) \in(-\delta, \delta) \times\left(Q_{m}-\delta, Q_{m}+\delta\right) .
$$

As Kirchhoff ellipses are exact solutions for $\varepsilon=0$, we obtain

$$
\varphi(0, Q, 0)=0, \quad \forall Q \in\left(Q_{m}-\delta, Q_{m}+\delta\right) .
$$

The equation of $F_{2}$ in a neighbourhood of $\left(0, Q_{m}, 0,0\right)$ takes the form

$$
\widehat{F_{2}}(\varepsilon, Q, s) \triangleq \Pi_{2} F\left(\varepsilon, Q, s v_{m}+\varphi(\varepsilon, Q, s)\right)=0, \quad \forall|\varepsilon|,\left|Q-Q_{m}\right|,|s|<\delta .
$$

From the relations (6.12) and (6.13) we deduce that

$$
\widehat{F_{2}}(\varepsilon, Q, 0)=0, \quad \forall|\varepsilon| \leq \delta, \forall\left|Q-Q_{m}\right| \leq \delta .
$$

Set

$$
\widehat{g}(\varepsilon, Q, s) \triangleq\left\{\begin{array}{l}
\widehat{F_{2}(\varepsilon, Q, s)}, \quad s \neq 0 \\
\Pi_{2} \partial_{f} F(\varepsilon, Q, \varphi(\varepsilon, Q, 0))\left(v_{m}+\partial_{s} \varphi(\varepsilon, Q, 0)\right), \quad s=0 .
\end{array}\right.
$$

Then the function $\widehat{g}$ is continuous and

$$
\begin{aligned}
\widehat{g}\left(0, Q_{m}, 0\right) & =\Pi_{2} \partial_{f} F\left(0, Q_{m}, 0\right)\left(v_{m}+\partial_{s} \varphi\left(0, Q_{m}, 0\right)\right) \\
& =0
\end{aligned}
$$

Indeed, by differentiating with respect to $s$ the following equation

$$
F_{1}(\varepsilon, Q, s, \varphi(\varepsilon, Q, s))=0, \forall|\varepsilon|,\left|Q-Q_{m}\right|,|s| \leq \delta
$$

at the point $\left(0, Q_{m}, 0\right)$, we find

$$
\left(\mathrm{Id}-\Pi_{2}\right) \partial_{f} F\left(0, Q_{m}, 0\right)\left(v_{m}+\partial_{s} \varphi\left(0, Q_{m}, 0\right)\right)=0 .
$$

Consequently, $\partial_{s} \varphi\left(0, Q_{m}, 0\right) \in \operatorname{Ker}\left(\mathcal{L}_{Q_{m}}\right) \cap \mathcal{X}$, and therefore

$$
\partial_{s} \varphi\left(0, Q_{m}, 0\right)=0 \text {. }
$$

Thanks to (6.14), we obtain

$$
\partial_{Q} \varphi\left(0, Q_{m}, 0\right)=0
$$

Moreover, $\widehat{g}$ is differentiable with respect to $Q$ and

$$
\begin{aligned}
\partial_{Q} \widehat{g}\left(0, Q_{m}, 0\right) & =\Pi_{2} \partial_{Q} \partial_{f} F\left(0, Q_{m}, 0\right)\left(v_{m}+\partial_{s} \varphi\left(0, Q_{m}, 0\right)\right) \\
& +\Pi_{2} \partial_{f} F\left(0, Q_{m}, 0\right)\left(\partial_{Q} v_{m \mid Q=Q_{m}}+\partial_{Q} \partial_{s} \varphi\left(0, Q_{m}, 0\right)\right) \\
& +\Pi_{2} \partial_{f}^{2} F\left(0, Q_{m}, 0\right)\left(v_{m}+\partial_{s} \varphi\left(0, Q_{m}, 0\right), \partial_{Q} \varphi\left(0, Q_{m}, 0\right)\right) \\
& =\Pi_{2} \partial_{Q} \partial_{f} F\left(0, Q_{m}, 0\right)\left(v_{m}\right) .
\end{aligned}
$$

From the transversality assumption proved in Proposition (6.1), we obtain

$$
\partial_{Q} \widehat{g}\left(0, Q_{m}, 0\right) \neq 0 \text {. }
$$


Hence using a weak version of the implicit function theorem, see [5], we deduce that the solutions of $\widehat{g}(\varepsilon, Q, s)=0$ near the point $\left(0, Q_{m}, 0\right)$ are parametrized by a continuous surface $\gamma:\left(-\varepsilon_{0}, \varepsilon_{0}\right)^{2} \rightarrow \mathbb{R}$ such that $Q=\gamma(\varepsilon, s)$ and

$$
\widehat{g}(\varepsilon, \gamma(\varepsilon, s), s)=0, \forall|\varepsilon| \leq \varepsilon_{0}, \forall|s| \leq \varepsilon_{0}, \quad \text { with } \quad \varepsilon_{0}>0 .
$$

Therefore the solutions of the equation $F(\varepsilon, Q, f)=0$ near the point $\left(0, Q_{m}, 0\right)$ are given by the union $\mathcal{C}_{1} \cup \mathcal{C}_{2}$ where

$$
\mathcal{C}_{1}=\left\{(\varepsilon, Q, \varphi(\varepsilon, Q, 0)),|\varepsilon| \leq \varepsilon_{0},\left|Q-Q_{m}\right| \leq \varepsilon_{0}\right\}
$$

corresponding to the two-fold V-states constructed in Theorem 6.3 and

$$
\mathcal{C}_{2}=\left\{\left(\varepsilon, \gamma(\varepsilon, s), s v_{m}+\varphi(\varepsilon, \gamma(\varepsilon, s), s)\right),|\varepsilon| \leq \varepsilon_{0},|s| \leq \varepsilon_{0}\right\} .
$$

Note that the curve $\mathcal{C}_{2}$ is different from $\mathcal{C}_{1}$ since for $s \neq 0$ the $\mathrm{V}$-state parametrized by

$$
w \in \mathbb{T} \mapsto w+Q \bar{w}+s v_{m}(w)+\varphi(\varepsilon, \gamma(\varepsilon, s), s)
$$

is not two-fold because $m$ is odd and therefore $v_{m}$ is a non-vanishing even function. In addition the curve $\mathcal{C}_{2}$ intersects $\mathcal{C}_{1}$ at $s=0$. This achieves the proof.

6.5. Breakdown of the bifurcation diagram close to the resonant set. The numerical study conducted in Section 3 shows that, contrary to what occurs in the Euler equations, the two-fold branch is never connected for small $\varepsilon$ and is split into countable disjoint connected components or branches. The separation of the singularity set seems to happen around the resonant set $\mathcal{S}_{\text {reso }}=\left\{Q_{2 m}, m \geq 2\right\}$ due to the resonance between branches with the same symmetry. We provide an analytical confirmation of this behavior only around the point $Q_{4}$ which is more tractable than the remaining cases $Q_{2 m}, m \geq 3$. We point out that the separation of the two-fold branch around $Q_{4}$ is only proved locally in the bifurcation diagram. The global structure of this separation is much more complicated and may require more elaborate tools.

More precisely, we prove the following result.

Theorem 6.5. Consider the $V$-state equation (6.3). There exists $\varepsilon_{0}>0$ such that for any $\varepsilon \in$ $\left(-\varepsilon_{0}, \varepsilon_{0}\right) \backslash\{0\}$ there exists $r_{\varepsilon}>0$ such that the set

$$
\left\{F(\varepsilon, Q, f)=0,\left|Q-Q_{4}\right|<r_{\varepsilon}, f \in X_{2},\|f\|_{C^{1+\alpha}}<r_{\varepsilon}\right\}
$$

is given by the union of two disjoint one-dimensional curves.

Proof. The proof is based on studying the local structure of the V-state equation (4.6) through the associated quadratic form. At first sight there is a logarithmic singularity in $\varepsilon$ at second order which could present difficulties for understanding the local structure. However, as shown below, this term may be combined with the rotation term and therefore it does not contribute at the nonlinear level. To show this, we first make some transformations using new unknowns. From (6.7) we may write for $x>0$ the expansion

$$
K_{0}^{\varepsilon}(x)=\psi(1)-\log (x)-\frac{\varepsilon^{2}}{4} x^{2} \log (x)+\frac{\varepsilon^{2}}{4}(\psi(2)-\log (|\varepsilon| / 2)) x^{2}+\varepsilon^{4} \log \varepsilon \mathcal{R}^{\varepsilon}(x)
$$


where $\mathcal{R}^{\varepsilon}$ is at least of class $C^{3}$ in the variable $x$ and analytic in the variable $\varepsilon$. It follows that

$$
\begin{aligned}
f_{\mathbb{T}} \Phi^{\prime}(\tau) K_{0}^{\varepsilon}(|\Phi(\tau)-\Phi(w)|) d \tau & =-f_{\mathbb{T}} \Phi^{\prime}(\tau) \log (|\Phi(\tau)-\Phi(w)|) d \tau \\
& +\frac{\varepsilon^{2}}{4}(\psi(2)-\log (|\varepsilon| / 2)) f_{\mathbb{T}} \Phi^{\prime}(\tau)|\Phi(\tau)-\Phi(w)|^{2} d \tau \\
& -\frac{\varepsilon^{2}}{4} f_{\mathbb{T}} \Phi^{\prime}(\tau) \log (|\Phi(\tau)-\Phi(w)|)|\Phi(\tau)-\Phi(w)|^{2} d \tau \\
& +\varepsilon^{4} \log |\varepsilon| f_{\mathbb{T}} \Phi^{\prime}(\tau) \mathcal{R}^{\varepsilon}(|\Phi(\tau)-\Phi(w)|) d \tau .
\end{aligned}
$$

Denote $\Gamma=\Phi(\mathbb{T})$; then it is simple to obtain, by a change of variables and the residue theorem,

$$
\begin{aligned}
f_{\mathbb{\pi}} \Phi^{\prime}(\tau)|\Phi(\tau)-\Phi(w)|^{2} d \tau & =f_{\Gamma}|\xi-\Phi(w)|^{2} d \xi \\
& =f_{\Gamma}\left(|\xi|^{2}-\Phi(w) \bar{\xi}\right) d \xi
\end{aligned}
$$

From Cauchy-Pompeiu's formula we find

$$
f_{\Gamma}|\xi|^{2} d \xi=\frac{1}{\pi} \int_{D} \xi d A
$$

and since the domain $D$ delimited by the curve $\Gamma$ is two-fold and centered at the origin then

$$
f_{\Gamma}|\xi|^{2} d \xi=0
$$

Hence

$$
f_{\mathbb{T}}|\Phi(\tau)-\Phi(w)|^{2} \Phi^{\prime}(\tau) d \tau=-\Phi(w) f_{\mathbb{T}} \overline{\Phi(\tau)} \Phi^{\prime}(\tau) d \tau
$$

Thus we obtain

$$
\begin{aligned}
f_{\mathbb{T}} K_{0}^{\varepsilon}(|\Phi(\tau)-\Phi(w)|) \Phi^{\prime}(\tau) d \tau & =-f_{\mathbb{T}} \log (|\Phi(\tau)-\Phi(w)|) \Phi^{\prime}(\tau) d \tau \\
& -\frac{\varepsilon^{2}}{4}(\psi(2)-\log (|\varepsilon| / 2)) \Phi(w) f_{\mathbb{T}} \overline{\Phi(\tau)} \Phi^{\prime}(\tau) d \tau \\
& -\frac{\varepsilon^{2}}{4} f_{\mathbb{T}} \log (|\Phi(\tau)-\Phi(w)|)|\Phi(\tau)-\Phi(w)|^{2} \Phi^{\prime}(\tau) d \tau \\
& +\varepsilon^{4} \log |\varepsilon| f_{\mathbb{T}} \mathcal{R}^{\varepsilon}(|\Phi(\tau)-\Phi(w)|) \Phi^{\prime}(\tau) d \tau
\end{aligned}
$$

Inserting this identity into (4.6) and using (4.7) we find

$$
G(\varepsilon, \Omega, \Phi)=-G_{E}\left(\Omega_{\varepsilon}, \Phi\right)+\frac{\varepsilon^{2}}{4} \operatorname{Im}\left\{G_{1}(\Phi)\right\}+\varepsilon^{4} \log |\varepsilon| \operatorname{Im}\left\{G_{2}(\varepsilon, \Phi)\right\}
$$

with

and

$$
\begin{gathered}
\Omega_{\varepsilon} \triangleq \Omega+\frac{\varepsilon^{2}}{4}(\psi(2)-\log (|\varepsilon| / 2)) f_{\mathbb{T}} \overline{\Phi(\tau)} \Phi^{\prime}(\tau) d \tau \\
G_{1}(\Phi) \triangleq \bar{w} \overline{\Phi^{\prime}(w)} f_{\mathbb{T}} \log (|\Phi(\tau)-\Phi(w)|)|\Phi(\tau)-\Phi(w)|^{2} \Phi^{\prime}(\tau) d \tau
\end{gathered}
$$

$$
G_{2}(\varepsilon, \Phi) \triangleq-\bar{w} \overline{\Phi^{\prime}(w)} f_{\mathbb{T}} \Phi_{36}^{\prime}(\tau) \mathcal{R}^{\varepsilon}(|\Phi(\tau)-\Phi(w)|) d \tau
$$


As before we look for solutions of the form

$$
\Phi=\alpha_{Q}+f, f \in X_{2} .
$$

Note that the space $X_{2}$ was introduced previously in (6.8). We now impose the constraint

$$
\Omega_{\varepsilon}=\frac{1-Q^{2}}{4} .
$$

Set

$$
F_{E}(Q, f) \triangleq-G_{E}\left(\left(1-Q^{2}\right) / 4, \alpha_{Q}+f\right), \quad F_{1}(Q, f) \triangleq G_{1}\left(\alpha_{Q}+f\right)
$$

and

$$
F_{2}(\varepsilon, Q, f) \triangleq G_{2}\left(\varepsilon, \alpha_{Q}+f\right) .
$$

Then the $\mathrm{V}$-state equation is equivalent to

$$
\widehat{F}(\varepsilon, Q, f) \triangleq F_{E}(Q, f)+\frac{\varepsilon^{2}}{4} \operatorname{Im}\left\{F_{1}(Q, f)\right\}+\varepsilon^{4} \log (|\varepsilon|) \operatorname{Im}\left\{F_{2}(\varepsilon, Q, f)\right\}=0 .
$$

Following the same lines of Proposition 6.2 we can check that

$$
\begin{aligned}
\widehat{F}:(-1,1) \times(0, \mu) \times B_{r_{\mu}} & \longrightarrow Y_{2} \\
(\varepsilon, Q, f) & \longmapsto \widehat{F}(\varepsilon, Q, f)
\end{aligned}
$$

is well-defined and of class $C^{1}$, where the ball $B_{r_{\mu}}$ was previously defined in (6.10). In fact, we can easily check that $\widehat{F}$ is at least $C^{3}$. Moreover, $\partial_{Q} \widehat{F}$ and $D_{f} \widehat{F}$ are also $C^{3}$. Note that from (6.3) we have

$$
D_{f} \widehat{F}(0, Q, 0)=D_{f} F_{E}(Q, 0)=\mathcal{L}_{Q}
$$

and the full structure of $\mathcal{L}_{Q}$ was previously detailed in Proposition 6.1. Recall in particular that for $Q=Q_{4}$ one has

and

$$
\operatorname{Ker} \mathcal{L}_{Q_{4}}=\left\langle v_{4}\right\rangle, v_{4}(w)=\frac{w^{5}}{1-Q_{4} w^{2}}
$$

$$
R\left(\mathcal{L}_{Q_{4}}\right)=\left\{g \in C^{\alpha}(\mathbb{T}), g=\sum_{\substack{n \geq 1 \\ n \neq 2}} g_{n} e_{2 n}, \quad g_{n} \in \mathbb{R}\right\} .
$$

Let $\widehat{X}_{2}$ be a complement of $v_{4}$ in $X_{2}$, that is,

$$
X_{2}=\widehat{X}_{2} \oplus\left\langle v_{4}\right\rangle .
$$

Thus any $f \in X_{2}$ admits a unique decomposition in the form $f=s v_{4}+g$ with $g \in \widehat{X}_{2}$ and $s \in \mathbb{R}$. Denote by $\widehat{Y}_{2}$ the space $R\left(\mathcal{L}_{Q_{4}}\right)$, then

$$
Y_{2}=\widehat{Y}_{2} \oplus\left\langle e_{4}\right\rangle
$$

and let $\Pi: Y_{2} \rightarrow\left\langle e_{4}\right\rangle$ be the canonical projection along $\widehat{Y}_{2}$. Thus equation (6.16) is equivalent to (6.17) $H_{1}(\varepsilon, Q, s, g) \triangleq(\mathrm{Id}-\Pi) \widehat{F}\left(\varepsilon, Q, s v_{4}+g\right)=0 \quad$ and $\quad H_{2}(\varepsilon, Q, s, g) \triangleq \Pi \widehat{F}\left(\varepsilon, Q, s v_{4}+g\right)=0$. Now we can directly observe that

$$
\partial_{g} H_{1}\left(0, Q_{4}, 0,0\right)=(\mathrm{Id}-\Pi) \mathcal{L}_{Q_{4}},
$$

and that $\partial_{g} H_{1}\left(0, Q_{4}, 0,0\right): \widehat{X}_{2} \rightarrow \widehat{Y}_{2}$ is an isomorphism. Thus, by the implicit function theorem, the solutions of the equation $H_{1}(\varepsilon, Q, s, g)=0$ are described near the point $\left(0, Q_{4}, 0,0\right)$ by the parametrization $g=\varphi(\varepsilon, Q, s)$ with

$$
\varphi:(-\delta, \delta) \times\left(Q_{4}-\delta, Q_{4}+\delta\right) \times(-\delta, \delta) \rightarrow \widehat{X}_{2}, \quad \delta>0
$$


being a $C^{1}$ function. As the functions defining the $\mathrm{V}$-states are smooth enough, $\varphi$ is in fact at least of class $C^{3}$. Notice that the defect of regularity comes only from the variable $\varepsilon$ and thus one gets more smoothness in the remaining variables. Indeed, we have the following.

Remark 6.6. The functions $\partial_{Q} \varphi$ and $\partial_{s} \varphi$ are at least $C^{3}$. To prove this, we just differentiate the equation

$$
(\mathrm{Id}-\Pi) \widehat{F}\left(\varepsilon, Q, s v_{4}+\varphi(\varepsilon, Q, s)\right)=0, \forall(\varepsilon, Q, s) \in(-\delta, \delta) \times\left(Q_{4}-\delta, Q_{4}+\delta\right) \times(-\delta, \delta)
$$

and argue by induction.

Now since the ellipses are solutions, then by uniqueness we obtain

$$
\varphi(0, Q, 0)=0, \forall Q \in\left(Q_{4}-\delta, Q_{4}+\delta\right) .
$$

This implies that for any $k \in\{1,2,3\}$

$$
\partial_{Q}^{k} \varphi(0, Q, 0)=0, \forall Q \in\left(Q_{4}-\delta, Q_{4}+\delta\right) .
$$

On the other hand differentiating the first equation in (6.17) with respect to $s$ we obtain

$$
(\mathrm{Id}-\Pi)\left(\mathcal{L}_{Q_{4}}\left(v_{4}+\partial_{s} \varphi\left(0, Q_{4}, 0\right)\right)=0\right.
$$

and as $\partial_{s} \varphi\left(0, Q_{4}, 0\right) \in \widehat{X}_{2}$ we deduce

$$
\partial_{s} \varphi\left(0, Q_{4}, 0\right)=0 \text {. }
$$

Similarly, differentiating the first equation of (6.17) with respect to $\varepsilon$, we obtain (due to (6.16))

$$
(\mathrm{Id}-\Pi) \mathcal{L}_{Q_{4}} \partial_{\varepsilon} \varphi\left(0, Q_{4}, 0\right)=0
$$

which implies that

$$
\partial_{\varepsilon} \varphi\left(0, Q_{4}, 0\right)=0 .
$$

Now the V-state equation reduces in this small neighbourhood to the finite-dimensional equation

$$
\psi(\varepsilon, Q, s) \triangleq H_{2}(\varepsilon, Q, s, \varphi(\varepsilon, Q, s))=0 .
$$

It is obvious from (6.16) that

$$
\psi(\varepsilon, Q, s)=\psi_{E}(\varepsilon, Q, s)+\frac{\varepsilon^{2}}{4} \psi_{1}(\varepsilon, Q, s)+\varepsilon^{4} \log |\varepsilon| \psi_{2}(\varepsilon, Q, s)
$$

with

$$
\psi_{E}(\varepsilon, Q, s) \triangleq \Pi F_{E}\left(Q, s v_{4}+\varphi(\varepsilon, s, Q)\right), \psi_{1}(\varepsilon, Q, s) \triangleq \Pi \operatorname{Im}\left\{F_{1}\left(Q, s v_{4}+\varphi(\varepsilon, Q, s)\right)\right\}
$$

and

Moreover,

$$
\psi_{2}(\varepsilon, Q, s) \triangleq \Pi \operatorname{Im}\left\{F_{2}\left(\varepsilon, Q, s v_{4}+\varphi(\varepsilon, Q, s)\right)\right\}
$$

which implies that for any $k \in\{1,2,3,4\}$

$$
\partial_{Q}^{k} \psi(0, Q, 0)=0, \forall Q \in\left(Q_{4}-\delta, Q_{4}+\delta\right) .
$$

Moreover straightforward computations yield, in view of (6.22) and the structures of $\Pi$ and $\mathcal{L}_{Q_{4}}$,

$$
\partial_{\varepsilon} \psi_{E}\left(0, Q_{4}, 0\right)=\Pi \mathcal{L}_{Q_{4}} \partial_{\varepsilon} \varphi\left(0, Q_{4}, 0\right)=0 .
$$

Using once again (6.22) we find

$$
\begin{aligned}
\partial_{\varepsilon}^{2} \psi_{E}\left(0, Q_{4}, 0\right) & =\Pi \partial_{f}^{2} F_{E}\left(Q_{4}, 0\right)\left(\partial_{\varepsilon} \varphi\left(0, Q_{4}, 0\right), \partial_{\varepsilon} \varphi\left(0, Q_{4}, 0\right)\right)+\Pi \mathcal{L}_{Q_{4}} \partial_{\varepsilon}^{2} \varphi\left(0, Q_{4}, 0\right) \\
& =0
\end{aligned}
$$


Hence we obtain

$$
\begin{aligned}
\partial_{\varepsilon}^{2} \psi\left(0, Q_{4}, 0\right) & =\frac{1}{2} \psi_{1}\left(0, Q_{4}, 0\right) \\
& =\frac{1}{2} \Pi \operatorname{Im}\left\{F_{1}\left(Q_{4}, 0\right)\right\}
\end{aligned}
$$

To compute the projection, we need to calculate the coefficients of $w^{4}$ and $\bar{w}^{4}$ in the Fourier expansion of $F_{1}\left(Q_{4}, 0\right)$. First we have

$$
\begin{aligned}
F_{1}\left(Q_{4}, 0\right) & =\bar{w} \overline{\alpha_{Q_{4}}^{\prime}(w)} I(w) \\
& =\left(\bar{w}-Q_{4} w\right) I(w)
\end{aligned}
$$

with

$$
I(w) \triangleq f_{\mathbb{T}} \log \left(\left|\alpha_{Q_{4}}(\tau)-\alpha_{Q_{4}}(w)\right|\right)\left|\alpha_{Q_{4}}(\tau)-\alpha_{Q_{4}}(w)\right|^{2} \alpha_{Q_{4}}^{\prime}(\tau) d \tau .
$$

Using the identity

$$
\left|\alpha_{Q_{4}}(\tau)-\alpha_{Q_{4}}(w)\right|=|\tau-w|\left|1-Q_{4} \tau w\right|, \quad \forall \tau, w \in \mathbb{T}
$$

we find after straightforward computations

$$
\left|\alpha_{Q_{4}}(\tau)-\alpha_{Q_{4}}(w)\right|^{2} \alpha_{Q_{4}}^{\prime}(\tau)=\sum_{k=0}^{2} \alpha_{k}(w) \tau^{k}+\sum_{k=1}^{4} \beta_{k}(w) \bar{\tau}^{k}
$$

with

$$
\begin{gathered}
\alpha_{0}(w) \triangleq 2+Q_{4}^{2}+Q_{4}\left(w^{2}+\bar{w}^{2}\right), \quad \alpha_{1}(w) \triangleq-2 Q_{4} w-\left(1+Q_{4}^{2}\right) \bar{w}, \quad \alpha_{2}(w) \triangleq Q_{4}, \\
\beta_{1}(w) \triangleq\left(Q_{4}^{2}-1\right)\left(Q_{4} \bar{w}+w\right), \quad \beta_{2}(w) \triangleq-Q_{4}\left(2 Q_{4}^{2}+1\right)-Q_{4}^{2} w^{2}-Q_{4}^{2} \bar{w}^{2}, \\
\beta_{3}(w) \triangleq 2 Q_{4}^{2} \bar{w}+Q_{4}\left(1+Q_{4}^{2}\right) w \quad \text { and } \quad \beta_{4}(w) \triangleq-Q_{4}^{2} .
\end{gathered}
$$

Now we compute for $n \in \mathbb{Z}$

$$
\begin{aligned}
f_{\mathbb{T}} \log \left(\left|\alpha_{Q_{4}}(\tau)-\alpha_{Q_{4}}(w)\right| \tau^{n} d \tau\right. & =f_{\mathbb{T}} \log (|\tau-w|) \tau^{n} d \tau+f_{\mathbb{T}} \log \left(\left|1-Q_{4} \tau w\right|\right) \tau^{n} d \tau \\
& \triangleq I_{n}(w)+J_{n}(w) .
\end{aligned}
$$

First note that by a change of variables

$$
I_{n}(w)=w^{n+1} \frac{1}{2 \pi} \int_{0}^{2 \pi} \log \left|1-e^{i \theta}\right| e^{i(n+1) \theta} d \theta .
$$

From elementary trigonometric identities we write

$$
I_{n}(w)=w^{n+1} \frac{1}{4 \pi} \int_{0}^{2 \pi} \log \left(4 \sin ^{2}(\theta / 2)\right) e^{i(n+1) \theta} d \theta .
$$

Using Lemma A.3 of [3] we get

$$
I_{n}(w)=\left\{\begin{array}{l}
-\frac{1}{2|n+1|} w^{n+1}, \quad \text { if } \quad n \in \mathbb{Z} \backslash\{-1\} \\
0, \quad \text { if } n=-1 .
\end{array}\right.
$$


In addition

$$
\begin{aligned}
J_{n}(w) & =\bar{w}^{n+1} \frac{1}{2 \pi} \int_{0}^{2 \pi} \log \left|1-Q_{4} e^{i \theta}\right| e^{i(n+1) \theta} d \theta \\
& =\bar{w}^{n+1} \frac{1}{4 \pi} \int_{0}^{2 \pi} \log \left|1+Q_{4}^{2}-2 Q_{4} \cos (\theta)\right| e^{i(n+1) \theta} d \theta .
\end{aligned}
$$

Again from Lemma A.4 [3] we obtain

$$
J_{n}(w)=\left\{\begin{array}{l}
-\frac{1}{2|n+1|} Q_{4}^{|n+1|} \bar{w}^{n+1}, \quad \text { if } \quad n \in \mathbb{Z} \backslash\{-1\} \\
0, \quad \text { if } n=-1 .
\end{array}\right.
$$

Putting together the preceding identities one finds

$$
f_{\mathbb{T}} \log \left(\left|\alpha_{Q_{4}}(\tau)-\alpha_{Q_{4}}(w)\right| \tau^{n} d \tau=\left\{\begin{array}{l}
-\frac{1}{2|n+1|}\left(w^{n+1}+Q_{4}^{|n+1|} \bar{w}^{n+1}\right), \quad \text { if } \quad n \in \mathbb{Z} \backslash\{-1\} \\
0, \quad \text { if } n=-1 .
\end{array}\right.\right.
$$

Using (6.26) we find

$$
\begin{aligned}
I(w) & =\sum_{k=0}^{2} \alpha_{k}(w)\left(I_{k}(w)+J_{k}(w)\right)+\sum_{k=1}^{4} \beta_{k}(w)\left(I_{-k}(w)+J_{-k}(w)\right) \\
& =\sum_{k=0}^{2} \alpha_{k}(w)\left(I_{k}(w)+J_{k}(w)\right)+\sum_{k=2}^{4} \beta_{k}(w)\left(I_{-k}(w)+J_{-k}(w)\right) .
\end{aligned}
$$

From straightforward calculation, and using the fact that $Q_{4}$ is a solution of (6.5) with $m=4$, we obtain

$$
\Pi \operatorname{Im}\left\{\bar{w} \overline{\alpha_{Q_{4}}^{\prime}(w)} \sum_{k=0}^{2} \alpha_{k}\left(I_{k}+J_{k}\right)\right\}=\frac{Q_{4}^{2}}{12}\left(5-Q_{4}^{2}\right) e_{4}
$$

Similarly we obtain

$$
\Pi \operatorname{Im}\left\{\bar{w} \overline{\alpha_{Q_{4}}^{\prime}(w)} \sum_{k=2}^{4} \beta_{k}\left(I_{-k}+J_{-k}\right)\right\}=\frac{Q_{4}^{6}-3 Q_{4}^{4}-2 Q_{4}^{2}}{12} e_{4} .
$$

Consequently

$$
\Pi \operatorname{Im}\left\{F_{1}\left(Q_{4}, 0\right)\right\}=\frac{Q_{4}^{2}}{12}\left(Q_{4}^{4}-4 Q_{4}^{2}+3\right) e_{4}
$$

Therefore

$$
\partial_{\varepsilon}^{2} \psi\left(0, Q_{4}, t\right)=\frac{Q_{4}^{2}}{24}\left(Q_{4}^{4}-4 Q_{4}^{2}+3\right) e_{4} .
$$

We next compute $\partial_{s} \partial_{\varepsilon} \psi\left(0, Q_{4}, 0\right)$. From (6.24) we write

$$
\begin{aligned}
\partial_{s} \partial_{\varepsilon} \psi\left(0, Q_{4}, 0\right) & =\Pi \partial_{f}^{2} F_{E}\left(Q_{4}, 0\right)\left[\partial_{\varepsilon} \varphi\left(0, Q_{4}, 0\right), v_{4}+\partial_{s} \varphi\left(0, Q_{4}, 0\right)\right] \\
& +\Pi \mathcal{L}_{Q_{4}} \partial_{\varepsilon} \partial_{s} \varphi\left(0, Q_{4}, 0\right) .
\end{aligned}
$$

From (6.22) and the structure of $\Pi$ we find

$$
\partial_{s} \partial_{\varepsilon} \psi\left(0, Q_{4}, 0\right)=0
$$

Similarly we find

$$
\begin{aligned}
\partial_{Q} \partial_{s} \psi\left(0, Q_{4}, 0\right) & =\Pi \partial_{Q} \partial_{s} F_{E}\left(0, Q_{4}, 0\right) \\
& =\partial_{Q}\left\{\Pi \partial_{f} F_{E}(Q, \varphi(0, Q, 0))\left(v_{4}+\partial_{s} \varphi(0, Q, 0)\right)\right\}_{Q=Q_{4}}
\end{aligned}
$$


Using (6.19) and (6.20) we deduce that

$$
\partial_{Q} \partial_{s} \psi\left(0, Q_{4}, 0\right)=\Pi\left\{\partial_{Q} \mathcal{L}_{Q} v_{4}\right\}_{Q=Q_{4}} .
$$

This is the transversality assumption in the Crandall-Rabinowitz theorem. According to [15] we have

and thus

$$
\Pi\left\{\partial_{Q} \mathcal{L}_{Q} v_{4}\right\}_{Q=Q_{4}}=4\left(Q_{4}+Q_{4}^{3}\right) e_{4}
$$

$$
\partial_{Q} \partial_{s} \psi\left(0, Q_{4}, 0\right)=4\left(Q_{4}+Q_{4}^{3}\right) e_{4} .
$$

To compute $\partial_{Q} \partial_{\varepsilon} \psi\left(0, Q_{4}, 0\right)$ we note from (6.19), (6.22) and the identity $\Pi \mathcal{L}_{Q_{4}}=0$ that

$$
\begin{aligned}
\partial_{Q} \partial_{\varepsilon} \psi\left(0, Q_{4}, 0\right) & =\Pi \partial_{Q}\left\{\partial_{f} F_{E}(Q, \varphi(0, Q, 0)) \partial_{\varepsilon} \varphi(0, Q, 0)\right\}_{Q=Q_{4}} \\
& =0 .
\end{aligned}
$$

The computations of $\partial_{s}^{2} \psi\left(0, Q_{4}, 0\right)$ can be performed using the formula

$$
\begin{aligned}
\partial_{s}^{2} \psi\left(0, Q_{4}, 0\right) & =\partial_{f}^{2} F_{E}\left(Q_{4}, 0\right)\left[v_{4}, v_{4}\right] \\
& =\left.\left\{\frac{d^{2}}{d s^{2}} F_{E}\left(Q_{4}, s v_{4}\right)\right\}\right|_{s=0} .
\end{aligned}
$$

Observe from (4.7) that

$$
\begin{aligned}
F_{E}\left(Q_{4}, s v_{4}\right) & =G\left(0,\left(1-Q_{4}^{2}\right) / 4, \alpha_{Q_{4}}+s v_{4}\right) \\
& =-G_{E}\left(\left(1-Q_{4}^{2}\right) / 4, \alpha_{Q_{4}}+s v_{4}\right)
\end{aligned}
$$

with

$$
\begin{gathered}
G_{E}\left(1-Q_{4}^{2} / 4, \alpha_{Q_{4}}+s v_{4}\right)=\operatorname{Im}\left\{\left(\frac{1-Q_{4}^{2}}{4}\left(\overline{\alpha_{Q_{4}}(w)}+s \overline{v_{4}(w)}\right)+I(s)\right) w\left(\alpha_{Q_{4}}^{\prime}(w)+s v_{4}^{\prime}(w)\right)\right\}, \\
I(s) \triangleq \frac{1}{2} f_{\mathbb{T}} \frac{\bar{A}+s \bar{B}}{A+s B}\left(\alpha_{Q_{4}}^{\prime}(\tau)+s v_{4}^{\prime}(\tau)\right) d \tau
\end{gathered}
$$

and

$$
\begin{aligned}
A & =\alpha_{Q_{4}}(\tau)-\alpha_{Q_{4}}(w) \\
B & =v_{4}(\tau)-v_{4}(w) .
\end{aligned}
$$

It is easy to obtain

$$
\begin{aligned}
\left\{\frac{d^{2}}{d s^{2}} G_{E}\left(1-Q_{4}^{2} / 4, \alpha_{Q_{4}}+s v_{4}\right)\right\}_{s=0} & =\frac{1-Q_{4}^{2}}{2} \operatorname{Im}\left\{\overline{v_{4}(w)} w v_{4}^{\prime}(w)\right\}+\operatorname{Im}\left\{2 I^{\prime}(0) w v_{4}^{\prime}(w)+I^{\prime \prime}(0) w \alpha_{Q_{4}}^{\prime}(w)\right\} \\
& \triangleq \frac{1-Q_{4}^{2}}{2} \operatorname{Im}\left\{I_{1}(w)\right\}+\operatorname{Im}\left\{I_{2}(w)\right\}+\operatorname{Im}\left\{I_{3}(w)\right\} .
\end{aligned}
$$

We start by computing $\Pi \operatorname{Im}\left\{I_{1}(w)\right\}$. It is straightforward to show

$$
\overline{v_{4}(w)} w v_{4}^{\prime}(w)=\frac{w^{2}}{w^{2}-Q_{4}} \frac{-3 Q_{4} w^{2}+5}{\left(1-Q_{4} w^{2}\right)^{2}} .
$$

Set $z=w^{2}$. Then

$$
\overline{v_{4}(w)} w v_{4}^{\prime}(w)=\frac{z}{z-Q_{4}} \frac{-3 Q_{4} z+5}{\left(1-Q_{4} z\right)^{2}} .
$$

Note that $z \mapsto \frac{z}{z-Q_{4}} \frac{-3 Q_{4} z+5}{\left(1-Q_{4} z\right)^{2}}$ is holomorphic in the annulus of small radius $Q_{4}$ and large radius $\frac{1}{Q_{4}}$, and therefore it admits a Laurent expansion in this domain. To evaluate $\Pi \operatorname{Im}\left\{I_{1}(w)\right\}$ it suffices to 
compute the coefficients of $z^{2}$ and $\frac{1}{z^{2}}$ in that expansion using the residue theorem. The coefficient of $z^{2}$ is given by

$$
a \triangleq f_{\mathbb{T}} \frac{1}{z-Q_{4}} \frac{-3 Q_{4} z+5}{\left(1-Q_{4} z\right)^{2}} \frac{d z}{z^{2}} .
$$

Using the change of variable $z \mapsto \frac{1}{z}$ we obtain

$$
\begin{aligned}
a & =f_{\mathbb{T}} \frac{z^{2}}{1-Q_{4} z} \frac{5 z-3 Q_{4}}{\left(z-Q_{4}\right)^{2}} d z \\
& \triangleq f_{\mathbb{T}} \frac{g(z)}{\left(z-Q_{4}\right)^{2}} d z \\
& =g^{\prime}\left(Q_{4}\right)
\end{aligned}
$$

with

Thus we obtain,

$$
g(z) \triangleq \frac{z^{2}\left(5 z-3 Q_{4}\right)}{1-Q_{4} z}
$$

$$
a=\frac{-7 Q_{4}^{4}+9 Q_{4}^{2}}{\left(1-Q_{4}^{2}\right)^{2}}
$$

Now we move on to the coefficient of $\frac{1}{z^{2}}$ given by the formula

$$
b \triangleq f_{\mathbb{T}} \frac{z^{2}}{z-Q_{4}} \frac{-3 Q_{4} z+5}{\left(1-Q_{4} z\right)^{2}} d z .
$$

Using the residue theorem we obtain

$$
b=Q_{4}^{2} \frac{-3 Q_{4}^{2}+5}{\left(1-Q_{4}^{2}\right)^{2}} .
$$

Consequently

$$
\begin{aligned}
\frac{1-Q_{4}^{2}}{2} \Pi \operatorname{Im}\left\{I_{1}(w)\right\} & =\frac{1-Q_{4}^{2}}{2}(a-b) e_{4} \\
& =2 Q_{4}^{2} e_{4} .
\end{aligned}
$$

Next we compute $\Pi \operatorname{Im}\left\{I_{2}(w)\right\}$. First notice that

$$
2 I^{\prime}(0)=f_{\mathbb{T}} \frac{\bar{A}}{A} v_{4}^{\prime}(\tau) d \tau+f_{\mathbb{T}} \frac{A \bar{B}-B \bar{A}}{A^{2}} \alpha_{Q_{4}}^{\prime}(\tau) d \tau .
$$

We rewrite $I_{2}(w)$ as follows,

$$
I_{2}(w)=I_{21}(w)+I_{22}(w)+I_{23}(w),
$$

where

and

$$
\begin{gathered}
I_{21}(w)=w v_{4}^{\prime}(w) f_{\mathbb{T}} \frac{\bar{A}}{A} v_{4}^{\prime}(\tau) d \tau \\
I_{22}(w)=w v_{4}^{\prime}(w) f_{\mathbb{T}} \frac{\bar{B}}{A} \alpha_{Q_{4}}^{\prime}(\tau) d \tau
\end{gathered}
$$

$$
I_{23}(w)=-w v_{4}^{\prime}(w) f_{\mathbb{T}} \frac{B \bar{A}}{A^{2}} \alpha_{Q_{4}}^{\prime}(\tau) d \tau .
$$

Straightforward computations imply

$$
f_{\mathbb{T}} \frac{\bar{A}}{A} v_{4}^{\prime}(\tau) d \tau=\bar{w} f_{\mathbb{T}} \frac{Q_{4} w \tau-1}{\tau-Q_{4} \bar{w}} \frac{-3 Q_{4} \tau^{6}+5 \tau^{4}}{\left(1-Q_{4} \tau^{2}\right)^{2}} d \tau .
$$


Hence by the residue theorem we obtain

$$
f_{\mathbb{T}} \frac{\bar{A}}{A} v_{4}^{\prime}(\tau) d \tau=\bar{w}^{5}\left(Q_{4}^{2}-1\right) \frac{-3 Q_{4}^{7} \bar{w}^{2}+5 Q_{4}^{4}}{\left(1-Q_{4}^{3} \bar{w}^{2}\right)^{2}} .
$$

Since we can extend $z \mapsto I_{21}(z)$ to a holomorphic function in the annulus of small radius $Q$ and large radius $\frac{1}{Q_{4}}$, then $I_{21}$ admits a Laurent expansion in this domain. As before, to evaluate $\Pi \operatorname{Im}\left\{I_{21}(w)\right\}$ it suffices to compute the coefficients of $z^{4}$ and $\frac{1}{z^{4}}$ in that expansion using the residue theorem. The coefficient of $z^{4}$ is given by

$$
\tilde{a}=f_{\mathbb{T}} w v_{4}^{\prime}(w) \bar{w}^{5}\left(Q_{4}^{2}-1\right) \frac{-3 Q_{4}^{7} \bar{w}^{2}+5 Q_{4}^{4}}{\left(1-Q_{4}^{3} \bar{w}^{2}\right)^{2}} \frac{d w}{w^{5}} .
$$

Moreover, the coefficient of $\frac{1}{z^{4}}$ is given by

$$
\tilde{b}=f_{\mathbb{T}} w v_{4}^{\prime}(w) \bar{w}^{5}\left(Q_{4}^{2}-1\right) \frac{-3 Q_{4}^{7} \bar{w}^{2}+5 Q_{4}^{4}}{\left(1-Q_{4}^{3} \bar{w}^{2}\right)^{2}} w^{3} d w .
$$

One may thus deduce the following equality,

$$
\begin{aligned}
\Pi \operatorname{Im}\left\{I_{21}(w)\right\} & =(\tilde{a}-\tilde{b}) e_{4} \\
& =\frac{Q_{4}{ }^{6}\left(45-58 Q_{4}{ }^{4}+21 Q_{4}{ }^{8}\right)}{\left(Q_{4}{ }^{4}-1\right)\left(Q_{4}{ }^{2}+1\right)} e_{4} .
\end{aligned}
$$

As for $I_{22}$, using the change of variable $\tau \mapsto \bar{\tau}$, we obtain by the residue theorem

$$
\begin{aligned}
f_{\mathbb{T}} \frac{\bar{B}}{A} \alpha_{Q_{4}}^{\prime}(\tau) d \tau & =-\bar{w} f_{\mathbb{T}} \frac{v_{4}(\tau)-v_{4}(\bar{w})}{\tau-\bar{w}} \frac{1-Q_{4} \tau^{2}}{1-Q_{4} \bar{w} \tau} \frac{d \tau}{\tau} \\
& =-v_{4}(\bar{w}) .
\end{aligned}
$$

Again, we can extend $z \mapsto I_{21}(z)$ to a holomorphic function in the same annulus and thus we just need to compute the coefficients of $z^{4}$ and $\frac{1}{z^{4}}$ denoted by $\tilde{c}$ and $\tilde{d}$, respectively:

$$
\tilde{c}=-f_{\mathbb{T}} w v_{4}^{\prime}(w) v_{4}(\bar{w}) \frac{d w}{w^{5}}
$$

and

$$
\tilde{d}=-f_{\mathbb{T}} w v_{4}^{\prime}(w) v_{4}(\bar{w}) w^{3} d w .
$$

According to the residue theorem we obtain

$$
\begin{aligned}
\Pi \operatorname{Im}\left\{I_{22}(w)\right\} & =(\tilde{c}-\tilde{d}) e_{4} \\
& =\frac{4 Q_{4}{ }^{2}}{\left(Q_{4}{ }^{2}-1\right)} e_{4} .
\end{aligned}
$$

Now we move on to the last term $I_{23}(w)$. The computations are very tedious and we use the Maple symbolic manipulation package to obtain the following expressions

$$
-f_{\mathbb{T}} \frac{B \bar{A}}{A^{2}} \alpha_{Q_{4}}^{\prime}(\tau) d \tau=\frac{\sum_{i=0}^{5} \tilde{\alpha}_{2 i}\left(Q_{4}\right) w^{2 i}}{\omega^{3}\left(\omega^{2}-Q_{4}^{3}\right)\left(Q_{4}^{3}+Q_{4} \omega^{4}-\left(1+Q_{4}^{4}\right) \omega^{2}\right)}
$$

where

$$
\begin{gathered}
\tilde{\alpha}_{0}\left(Q_{4}\right) \triangleq 3 Q_{4}^{7}-4 Q_{4}^{9}, \quad \tilde{\alpha}_{2}\left(Q_{4}\right) \triangleq 4 Q_{4}^{10}-4 Q_{4}^{8}+7 Q_{4}^{6}-5 Q_{4}^{4}, \\
\tilde{\alpha}_{4}\left(Q_{4}\right) \triangleq Q_{4}^{9}-8 Q_{4}^{7}+7 Q_{4}^{5}-Q_{4}^{3}, \quad \tilde{\alpha}_{6}\left(Q_{4}\right) \triangleq Q_{4}^{8}-3 Q_{4}^{6}+3 Q_{4}^{4}-Q_{4}^{2}, \\
\tilde{\alpha}_{8}\left(Q_{4}\right) \triangleq 3 Q_{4}^{3}-2 Q_{4}^{5}-Q_{4} \quad \text { and } \quad \tilde{\alpha}_{10}\left(Q_{4}\right) \triangleq Q_{4}^{2}-1 .
\end{gathered}
$$


Let $\tilde{e}$ and $\tilde{f}$ be the coefficient of $z^{4}$ and $\frac{1}{z^{4}}$ in the Laurent expansion of $I_{23}(z)$. Again using Maple, we obtain

$$
\begin{aligned}
\Pi \operatorname{Im}\left\{I_{23}(w)\right\} & =(\tilde{e}-\tilde{f}) e_{4} \\
& =-\frac{Q_{4}^{2}\left(21 Q_{4}^{12}-65 Q_{4}^{8}-12 Q_{4}^{6}+47 Q_{4}^{4}+12 Q_{4}^{2}+5\right)}{\left(Q_{4}^{4}-1\right)\left(Q_{4}^{2}+1\right)} e_{4} .
\end{aligned}
$$

Now using (6.33), (6.34) and (6.35) we find

$$
\Pi \operatorname{Im}\left\{I_{2}(w)\right\}=\frac{1}{2} \frac{Q_{4}^{2}\left(7 Q_{4}^{4}-2 Q_{4}^{2}-1\right)}{\left(Q_{4}^{2}-1\right)} e_{4} .
$$

Now we compute $\operatorname{Im}\left\{I_{3}(w)\right\}$. First we notice that

$$
I^{\prime \prime}(0)=f_{\mathbb{T}} \frac{B^{2} \bar{A}-B \bar{B} A}{A^{3}} \alpha_{Q_{4}}^{\prime}(\tau) d \tau+f_{\mathbb{T}} \frac{\bar{B} A-B \bar{A}}{A^{2}} v_{4}^{\prime}(\tau) d \tau .
$$

Consequently, we can split $I_{3}$ as follows,

$$
I_{3}(w)=I_{31}(w)+I_{32}(w)+I_{33}(w)+I_{34}(w)
$$

where

and

$$
\begin{gathered}
I_{31}(w)=w \alpha_{Q_{4}}^{\prime}(w) f_{\mathbb{T}} \frac{B^{2} \bar{A}}{A^{3}} \alpha_{Q_{4}}^{\prime}(\tau) d \tau, \\
I_{32}(w)=w \alpha_{Q_{4}}^{\prime}(w) f_{\mathbb{T}} \frac{\bar{B}}{A} v_{4}^{\prime}(\tau) d \tau, \\
I_{33}(w)=-w \alpha_{Q_{4}}^{\prime}(w) f_{\mathbb{T}} \frac{B \bar{A}}{A^{2}} v_{4}^{\prime}(\tau) d \tau
\end{gathered}
$$

$$
I_{34}(w)=-w \alpha_{Q_{4}}^{\prime}(w) f_{\mathbb{T}} \frac{B \bar{B}}{A^{2}} \alpha_{Q_{4}}^{\prime}(\tau) d \tau
$$

In the following, we denote by $a_{3 i}$ and $b_{3 i}$ the coefficient in front of $z^{4}$ and $\frac{1}{z^{4}}$ in the Laurent expansion of $I_{3 i}(z)$ on the same annulus as before. We obtain the following expressions using Maple,

$$
a_{31}=-\frac{Q_{4}^{2}\left(-12 Q_{4}^{6}+8 Q_{4}^{2}+26 Q_{4}^{4}+3+12 Q_{4}^{12}+4 Q_{4}^{10}-33 Q_{4}^{8}\right)}{\left(Q_{4}^{4}-1\right)^{2}\left(Q_{4}^{2}+1\right)}
$$

and

$$
b_{31}=-\frac{Q_{4}^{6}\left(78 Q_{4}^{4}+8 Q_{4}^{2}+3+56 Q_{4}^{12}-129 Q_{4}^{8}-20 Q_{4}^{6}+12 Q_{4}^{10}\right)}{\left(Q_{4}^{4}-1\right)^{2}\left(Q_{4}^{2}+1\right)}
$$

Therefore,

$$
\begin{aligned}
\Pi \operatorname{Im}\left\{I_{31}(w)\right\} & =\left(a_{31}-b_{31}\right) e_{4} \\
& =\frac{\left(56 Q_{4}^{12}+12 Q_{4}^{10}-85 Q_{4}^{8}-12 Q_{4}^{6}+26 Q_{4}^{4}+8 Q_{4}^{2}+3\right) Q_{4}^{2}}{\left(Q_{4}^{2}+1\right)\left(-1+Q_{4}^{4}\right)} e_{4} .
\end{aligned}
$$

Similarly we obtain using Maple,

$$
f_{\mathbb{T}} \frac{\bar{B}}{A} v_{4}^{\prime}(\tau) d \tau=\frac{\sum_{i=0}^{5} \beta_{2 i}\left(Q_{4}\right) w^{2 i}}{w^{5}\left(w^{2}-Q_{4}^{3}\right)^{2}\left(w^{2}-Q_{4}\right)\left(Q_{4}^{2}-1\right)^{2}}
$$

where 


$$
\begin{gathered}
\beta_{0}\left(Q_{4}\right):=-6 Q_{4}^{9}+3 Q_{4}^{7}+3 Q_{4}^{11}, \quad \beta_{2}\left(Q_{4}\right):=3 Q_{4}^{10}-11 Q_{4}^{8}+13 Q_{4}^{6}-5 Q_{4}^{4}, \\
\beta_{4}\left(Q_{4}\right):=3 Q_{4}^{9}-11 Q_{4}^{7}+13 Q_{4}^{5}-5 Q_{4}^{3}, \quad \beta_{6}\left(Q_{4}\right):=-6 Q_{4}^{6}+13 Q_{4}^{4}-5 Q_{4}^{2}, \\
\beta_{8}\left(Q_{4}\right):=-Q_{4}^{5}+6 Q_{4}^{3}-5 Q_{4} \text { and } \beta_{10}\left(Q_{4}\right):=3 Q_{4}^{2}-5 .
\end{gathered}
$$

The coefficients in the Laurent expansion have the following expressions,

$$
a_{32}=0
$$

and

Consequently,

$$
b_{32}=\frac{\left(7 Q_{4}^{6}-9 Q_{4}^{4}+3 Q_{4}^{2}-5\right) Q_{4}^{2}}{\left(Q_{4}^{2}-1\right)^{2}}
$$

$$
\begin{aligned}
\Pi \operatorname{Im}\left\{I_{32}(w)\right\} & =\left(a_{32}-b_{32}\right) e_{4} \\
& =-\frac{\left(7 Q_{4}^{6}-9 Q_{4}^{4}+3 Q_{4}^{2}-5\right) Q_{4}^{2}}{\left(Q_{4}^{2}-1\right)^{2}} e_{4} .
\end{aligned}
$$

Thanks to Maple, one may find the following expression

$$
I_{33}(w)=\frac{\left(w^{2}-Q_{4}\right) Q_{4}^{4}\left(\sum_{i=0}^{6} \gamma_{2 i}\left(Q_{4}\right) w^{2 i}\right)}{w^{6}\left(w^{2}-Q_{4}^{3}\right)\left(-\left(Q_{4}^{10}+3 Q_{4}^{6}\right) w^{2}+3 Q_{4}^{3}\left(Q_{4}^{4}+1\right) w^{4}-\left(3 Q_{4}^{4}+1\right) w^{6}+Q_{4}^{9}+Q_{4} w^{8}\right)}
$$

where

$$
\begin{gathered}
\gamma_{0}\left(Q_{4}\right)=18 Q_{4}^{12}-15 Q_{4}^{10}, \quad \gamma_{2}\left(Q_{4}\right)=30 Q_{4}^{11}-68 Q_{4}^{9}+48 Q_{4}^{7}-18 Q_{4}^{13}, \\
\gamma_{4}\left(Q_{4}\right)=-15 Q_{4}^{12}-105 Q_{4}^{8}+90 Q_{4}^{6}+80 Q_{4}^{10}-45 Q_{4}^{4}, \quad \gamma_{6}\left(Q_{4}\right)=-12 Q_{4}^{11}-136 Q_{4}^{7}+122 Q_{4}^{5}+66 Q_{4}^{9}-40 Q_{4}^{3}, \\
\gamma_{8}\left(Q_{4}\right)=-35 Q_{4}^{2}+104 Q_{4}^{4}+46 Q_{4}^{8}-112 Q_{4}^{6}, \quad \gamma_{10}\left(Q_{4}\right)=-30 Q_{4}-64 Q_{4}^{5}+86 Q_{4}^{3},
\end{gathered}
$$

and

$$
\gamma_{12}\left(Q_{4}\right)=30 Q_{4}^{2}-25
$$

This allows one to obtain

$$
a_{33}=\frac{Q_{4}^{6}\left(9 Q_{4}^{8}-26 Q_{4}^{4}+25\right)}{\left(Q_{4}^{2}+1\right)\left(-1+Q_{4}^{4}\right)^{2}}
$$

and

$$
b_{33}=\frac{Q_{4}^{6}\left(-131 Q_{4}^{8}+56 Q_{4}^{12}+78 Q_{4}^{4}+5+12 Q_{4}^{2}-24 Q_{4}^{6}+12 Q_{4}^{10}\right)}{\left(Q_{4}^{2}+1\right)\left(-1+Q_{4}^{4}\right)^{2}} .
$$

Finally we obtain

$$
\begin{aligned}
\Pi \operatorname{Im}\left\{I_{33}(w)\right\} & =\left(a_{33}-b_{33}\right) e_{4} \\
& =-4 \frac{Q_{4}^{6}\left(14 Q_{4}^{8}+3 Q_{4}^{6}-21 Q_{4}^{4}-3 Q_{4}^{2}+5\right)}{\left(Q_{4}^{2}+1\right)\left(-1+Q_{4}^{4}\right)} e_{4} .
\end{aligned}
$$

For the last term, we use Maple to obtain

$$
-f_{\mathbb{T}} \frac{B \bar{B}}{A^{2}} \alpha_{Q_{4}}^{\prime}(\tau) d \tau=-\frac{\sum_{i=0}^{5} \xi_{2 i}\left(Q_{4}\right) w^{2 i}}{w^{5}\left(w^{2}-Q_{4}^{3}\right)\left(\left(Q_{4}^{4}+Q_{4}^{2}+1\right) w^{2}\left(Q_{4}-w^{2}\right)+Q_{4}\left(w^{6}-Q_{4}^{3}\right)\right)}
$$

where

$$
\begin{gathered}
\xi_{0}\left(Q_{4}\right)=-3 Q_{4}^{7}, \quad \xi_{2}\left(Q_{4}\right)=3 Q_{4}^{8}-3 Q_{4}^{6}+5 Q_{4}^{4}, \\
\xi_{4}\left(Q_{4}\right)=3 Q_{4}^{7}-8 Q_{4}^{5}+5 Q_{4}^{3}, \quad \xi_{6}\left(Q_{4}\right)=3 Q_{4}^{6}-8 Q_{4}^{4}+5 Q_{4}^{2}, \\
\xi_{8}\left(Q_{4}\right)=-6 Q_{4}^{3}+5 Q_{4} \text { and } \xi_{10}\left(Q_{4}\right)=2 Q_{4}^{2}+5 .
\end{gathered}
$$


Thus we deduce using Maple once again

$$
a_{34}=-\frac{Q_{4}^{2}\left(3 Q_{4}^{2}-5\right)}{\left(Q_{4}^{2}-1\right)^{2}}
$$

and

$$
b_{34}=-\frac{Q_{4}^{2}\left(4 Q_{4}^{6}-4 Q_{4}^{4}+3 Q_{4}^{2}-5\right)}{\left(Q_{4}^{2}-1\right)^{2}} .
$$

Therefore we find

$$
\begin{aligned}
\Pi \operatorname{Im}\left\{I_{34}(w)\right\} & =\left(a_{34}-b_{34}\right) e_{4} \\
& =\frac{4 Q_{4}^{6}}{\left(Q_{4}^{2}-1\right)} e_{4} .
\end{aligned}
$$

Finally, using (6.37), (6.38),(6.39) and (6.40) one finds

$$
\Pi \operatorname{Im}\left\{I_{3}(w)\right\}=-2 \frac{Q_{4}^{2}\left(2 Q_{4}^{6}-4 Q_{4}^{4}+Q_{4}^{2}-1\right)}{\left(-1+Q_{4}^{2}\right)^{2}} e_{4} .
$$

Now combining $(6.32), 6.36)$ with $(6.41)$ and simplifying the polynomial equation of $Q_{4}$, we obtain

$$
\partial_{t}^{2} \psi\left(0, Q_{4}, 0\right)=-\frac{Q_{4}^{2}\left(3 Q_{4}^{6}+Q_{4}^{4}-5 Q_{4}^{2}+5\right)}{\left(-1+Q_{4}^{2}\right)^{2}} e_{4}
$$

To summarize, up to this point we have proved that the $\mathrm{V}$-state equation (6.24) reduces in the small neighbourhood $I_{\delta} \triangleq(-\delta, \delta) \times\left(-\delta+Q_{4}, Q_{4}+\delta\right) \times(-\delta, \delta)$ to the finite-dimensional equation

$$
\psi(\varepsilon, Q, s)=0 \text {. }
$$

As $\psi$ is at least $\mathcal{C}^{3}$, we can use a Taylor expansion with the integral form for the remainder around the point $\left(0, Q_{4}, 0\right)$,

$$
\begin{aligned}
\psi(\varepsilon, Q, s) & =\psi\left(0, Q_{4}, 0\right)+s \partial_{s} \psi\left(0, Q_{4}, 0\right)+\varepsilon \partial_{\varepsilon} \psi\left(0, Q_{4}, 0\right)+\left(Q-Q_{4}\right) \partial_{Q} \psi\left(0, Q_{4}, 0\right)+\frac{s^{2}}{2} \partial_{s s}^{2} \psi\left(0, Q_{4}, 0\right) \\
& +\frac{\varepsilon^{2}}{2} \partial_{\varepsilon}^{2} \psi\left(0, Q_{4}, 0\right)+\frac{\left(Q-Q_{4}\right)^{2}}{2} \partial_{Q}^{2} \psi\left(0, Q_{4}, 0\right)+\varepsilon\left(Q-Q_{4}\right) \partial_{\varepsilon Q}^{2} \psi\left(0, Q_{4}, 0\right)+\varepsilon s \partial_{\varepsilon s}^{2} \psi\left(0, Q_{4}, 0\right) \\
& +\left(Q-Q_{4}\right) s \partial_{Q s}^{2} \psi\left(0, Q_{4}, 0\right)+\tilde{\varepsilon}(\varepsilon, Q, s) e_{4}
\end{aligned}
$$

where

$$
\tilde{\varepsilon}(\varepsilon, Q, s) e_{4}=\int_{0}^{1} \frac{(1-\theta)^{2}}{2 !} D^{3} \psi\left(\theta \varepsilon, Q_{4}+\theta\left(Q-Q_{4}\right), \theta s\right)\left(\varepsilon, Q-Q_{4}, s\right)^{3} d \theta .
$$

For a given vector $h$ we use the notation $h^{3}$ to denote $(h, h, h)$. Consequently, using the preceding computations concerning the quadratic expansion, we obtain for any $(\varepsilon, Q, s) \in I_{\delta}$

$$
\psi(\varepsilon, Q, s)=\left[a \varepsilon^{2}+b s\left(Q-Q_{4}\right)+c s^{2}+\tilde{\varepsilon}(\varepsilon, Q, s)\right] e_{4}
$$

with

and

$$
a=\frac{Q_{4}^{2}\left(Q_{4}^{4}-4 Q_{4}^{2}+3\right)}{48}, b=4 Q_{4}\left(Q_{4}^{2}+1\right)
$$

$$
c=-\frac{Q_{4}^{2}\left(3 Q_{4}^{6}+Q_{4}^{4}-5 Q_{4}^{2}+5\right)}{\left(-1+Q_{4}^{2}\right)^{2}} .
$$

Since $Q_{4}=\sqrt{\sqrt{2}-1}$, one may easily check that

$$
a>0, b>0 \text { and } c<0 \text {. }
$$


We introduce the parameters $\tilde{c}=-c, \tilde{b}=-\frac{b^{2}}{4 c}$ and $d=-\frac{b}{2 c}$, which are three positive constants, and write equation (6.42) in the new variables $\mathbf{Q}=Q-Q_{4}$ and $X=s-d \mathbf{Q}$. In this way, we find in a small neighbourhood of zero,

$$
\hat{\psi}(\varepsilon, \mathbf{Q}, X) \triangleq\left[a \varepsilon^{2}-\tilde{c} X^{2}+\tilde{b} \mathbf{Q}^{2}+\hat{\varepsilon}(\varepsilon, \mathbf{Q}, X)\right] e_{4}=0
$$

where

$$
\hat{\varepsilon}(\varepsilon, \mathbf{Q}, X)=\tilde{\varepsilon}(\varepsilon, Q, s) .
$$

For the quadratic equation

$$
a \varepsilon^{2}-\tilde{c} X^{2}+\tilde{b} \mathbf{Q}^{2}=0
$$

we find for given $\varepsilon \neq 0$ two disjoint curves

$$
X= \pm \sqrt{\frac{a}{\tilde{c}} \varepsilon^{2}+\frac{\tilde{b}}{\tilde{c}} \mathbf{Q}^{2}} .
$$

We prove that this structure persists for the full equation,

$$
a \varepsilon^{2}-\tilde{c} X^{2}+\tilde{b}^{2} \mathbf{Q}^{2}+\hat{\varepsilon}(\varepsilon, \mathbf{Q}, X)=0 .
$$

To this end, we check that the solutions have the form

$$
X=\sqrt{\frac{a}{\tilde{c}} \varepsilon^{2}+\frac{\tilde{b}}{\tilde{c}} \mathbf{Q}^{2}}+y
$$

where $y$ is a small correction described shortly below. From (6.44) we deduce that $y$ satisfies the equation

$$
G(\mathbf{Q}, y)=y,
$$

with

$$
G(\mathbf{Q}, y)=-\frac{y^{2}}{2 \sqrt{\frac{a}{\tilde{c}} \varepsilon^{2}+\frac{\tilde{b}}{\tilde{c}} \mathbf{Q}^{2}}}+\frac{\hat{\varepsilon}\left(\varepsilon, \mathbf{Q}, \sqrt{\frac{a}{\tilde{c}} \varepsilon^{2}+\frac{\tilde{b}}{\tilde{c}} \mathbf{Q}^{2}}+y\right)}{2 \tilde{c} \sqrt{\frac{a}{\tilde{c}} \varepsilon^{2}+\frac{\tilde{b}}{\tilde{c}} \mathbf{Q}^{2}}} .
$$

We prove the following lemma,

Lemma 6.7. There exist two strictly positive constants $\eta$ and $\varepsilon_{0}<1$ such that for any $0<\varepsilon \leq \varepsilon_{0}$

$$
\begin{aligned}
G: \bar{B}_{\eta \varepsilon^{\frac{5}{6}}} \times \bar{B}_{\eta \varepsilon^{\frac{3}{2}}} & \longrightarrow \bar{B}_{\eta \varepsilon^{\frac{3}{2}}} \\
(\mathbf{Q}, y) & \longmapsto G(\mathbf{Q}, y)
\end{aligned}
$$

is well-defined. Moreover, for any $\mathbf{Q} \in \bar{B}_{\eta \varepsilon^{\frac{5}{6}}}, G$ admits a unique fixed point $y(\mathbf{Q})$ which depends continuously on $\mathbf{Q}$.

Proof. We begin with the simple inequality,

$$
|G(\mathbf{Q}, y)| \leq \frac{y^{2}}{2 \sqrt{\frac{a}{\tilde{c}} \varepsilon^{2}+\frac{\tilde{b}}{\tilde{c}} \mathbf{Q}^{2}}}+\left|\frac{\hat{\varepsilon}\left(\varepsilon, \mathbf{Q}, \sqrt{\frac{a}{\tilde{c}} \varepsilon^{2}+\frac{\tilde{b}}{\tilde{c}} \mathbf{Q}^{2}}+y\right)}{2 \sqrt{\frac{a}{\tilde{c}} \varepsilon^{2}+\frac{\tilde{b}}{\tilde{c}} \mathbf{Q}^{2}}}\right| .
$$

It is plain that there exists $C>0$ such that,

$$
\frac{y^{2}}{2 \tilde{c} \sqrt{\frac{a}{\tilde{c}} \varepsilon^{2}+\frac{\tilde{b}}{\tilde{c}} \mathbf{Q}^{2}}} \leq C \eta^{2} \varepsilon^{2}
$$


For the second term we use the cubic form of the remainder and its continuity,

$$
\left|\hat{\varepsilon}\left(\varepsilon, \mathbf{Q}, \sqrt{\frac{a}{\tilde{c}} \varepsilon^{2}+\frac{\tilde{b}}{\tilde{c}} \mathbf{Q}^{2}}+y\right)\right| \leq C \eta^{3} \varepsilon^{\frac{5}{2}} .
$$

It follows that,

$$
|G(\mathbf{Q}, y)| \leq C \eta^{2} \varepsilon^{2}+C \eta^{3} \varepsilon^{\frac{3}{2}}
$$

Choosing $\eta$ such that

$$
C \eta+C \eta^{2} \leq 1
$$

we guarantee that $G$ is well-defined. In order to apply the Banach fixed point theorem with a parameter, we need only check that $G$ is a contraction. Let $y$ and $\tilde{y}$ be two elements of $\bar{B}_{\eta \varepsilon^{\frac{3}{2}}}$, then we have

$$
\begin{aligned}
|G(\mathbf{Q}, y)-G(\mathbf{Q}, \tilde{y})| & \leq\left|\frac{(y+\tilde{y})(\tilde{y}-y)}{2 \sqrt{\frac{a}{\tilde{c}} \varepsilon^{2}+\frac{\tilde{b}}{\tilde{c}} \mathbf{Q}^{2}}}\right| \\
& +\left|\frac{\hat{\varepsilon}\left(\varepsilon, \mathbf{Q}, \sqrt{\frac{a}{\tilde{c}} \varepsilon^{2}+\frac{\tilde{b}}{\tilde{c}} \mathbf{Q}^{2}}+y\right)-\hat{\varepsilon}\left(\varepsilon, \mathbf{Q}, \sqrt{\frac{a}{\tilde{c}} \varepsilon^{2}+\frac{\tilde{b}}{\tilde{c}} \mathbf{Q}^{2}}+\tilde{y}\right)}{2 \tilde{c} \sqrt{\frac{a}{\tilde{c}} \varepsilon^{2}+\frac{\tilde{b}}{\tilde{c}} \mathbf{Q}^{2}}}\right| .
\end{aligned}
$$

For the first term we write

$$
\left|\frac{(y+\tilde{y})(\tilde{y}-y)}{2 \sqrt{\frac{a}{\tilde{c}} \varepsilon^{2}+\frac{\tilde{b}}{\tilde{c}} \mathbf{Q}^{2}}}\right| \leq C \eta \varepsilon^{\frac{1}{2}}|\tilde{y}-y| .
$$

As for the second term we split it into two parts

$$
\hat{\varepsilon}\left(\varepsilon, \mathbf{Q}, \sqrt{\frac{a}{\tilde{c}} \varepsilon^{2}+\frac{\tilde{b}}{\tilde{c}} \mathbf{Q}^{2}}+y\right)-\hat{\varepsilon}\left(\varepsilon, \mathbf{Q}, \sqrt{\frac{a}{\tilde{c}} \varepsilon^{2}+\frac{\tilde{b}}{\tilde{c}} \mathbf{Q}^{2}}+\tilde{y}\right)=T_{1}(\varepsilon, \mathbf{Q}, \tilde{y}, y)+T_{2}(\varepsilon, \mathbf{Q}, \tilde{y}, y)
$$

with

$$
T_{1}(\varepsilon, \mathbf{Q}, \tilde{y}, y)=\int_{0}^{1} \frac{(1-\theta)^{2}}{2 !}\left[D^{3} \psi(\beta(\theta, \varepsilon, \mathbf{Q}, y))-D^{3} \psi(\beta(\theta, \varepsilon, \mathbf{Q}, \tilde{y}))\right][v(\varepsilon, \mathbf{Q}, y)]^{3} d \theta
$$

where

$$
\beta(\theta, \varepsilon, \mathbf{Q}, y)=\left(\theta \varepsilon, Q_{4}+\theta \mathbf{Q}, \theta\left(\sqrt{\frac{a}{\tilde{c}} \varepsilon^{2}+\frac{\tilde{b}}{\tilde{c}} \mathbf{Q}^{2}}+y+d \mathbf{Q}\right)\right), \quad v(\varepsilon, \mathbf{Q}, y)=\left(\begin{array}{c}
\varepsilon \\
\mathbf{Q} \\
\sqrt{\frac{a}{\tilde{c}} \varepsilon^{2}+\frac{\tilde{b}}{\tilde{c}} \mathbf{Q}^{2}}+y+d \mathbf{Q}
\end{array}\right)
$$

and

$$
T_{2}(\varepsilon, \mathbf{Q}, \tilde{y}, y)=\int_{0}^{1} \frac{(1-\theta)^{2}}{2 !}\left(D^{3} \psi(\beta(\theta, \varepsilon, \mathbf{Q}, \tilde{y}))[v(\varepsilon, \mathbf{Q}, y)]^{3} d \theta-D^{3} \psi(\beta(\theta, \varepsilon, \mathbf{Q}, \tilde{y}))[v(\varepsilon, \mathbf{Q}, \tilde{y})]^{3}\right) d \theta .
$$

According to Remark 6.6 and (6.23) one has that $\partial_{y} D^{3} \psi$ is continuous. This implies by virtue of the mean value theorem that

$$
\begin{aligned}
\left|T_{1}(\varepsilon, Q, \tilde{y}, y)\right| \leq C|y-\tilde{y}|\left(\varepsilon^{3}+|\mathbf{Q}|^{3}+|y|^{3}\right) \\
\leq C|y-\tilde{y}|\left(\varepsilon^{3}+\eta^{3} \varepsilon^{\frac{5}{2}}+\eta^{3} \varepsilon^{\frac{9}{2}}\right) \\
\leq C|y-\tilde{y}|\left(\varepsilon^{3}+\eta^{3} \varepsilon^{\frac{5}{2}}\right) .
\end{aligned}
$$


For the term $T_{2}$ we use the multi-linear structure of $D^{3} \psi$ which gives

$$
\begin{aligned}
\left|T_{2}(\varepsilon, Q, \tilde{y}, y)\right| & \leq C\left(\varepsilon^{2}+\mathbf{Q}^{2}+y^{2}\right)|y-\tilde{y}| \\
& \leq C\left(\varepsilon^{2}+\eta^{2} \varepsilon^{\frac{5}{3}}\right)|y-\tilde{y}| .
\end{aligned}
$$

Finally, we have the following inequality, since $\varepsilon, \eta \in[0,1]$,

$$
\begin{aligned}
|G(Q, y)-G(Q, \tilde{y})| & \leq C|y-\tilde{y}|\left(\varepsilon^{3}+\eta^{3} \varepsilon^{\frac{5}{2}}+\eta^{2} \varepsilon^{\frac{5}{3}}\right) \\
& \leq C|y-\tilde{y}|\left(\varepsilon^{3}+\eta^{2} \varepsilon^{\frac{5}{3}}\right) .
\end{aligned}
$$

For the choice of $\eta$ made before in (6.45), it suffices to fix $\varepsilon_{0}$ such that

$$
C\left(\varepsilon_{0}^{3}+\eta^{2} \varepsilon_{0}^{\frac{5}{3}}\right)<1
$$

in order to guarantee that $G$ is a contraction and hence admits a unique fixed point $y(\mathbf{Q}) \in \bar{B}_{\eta \varepsilon^{\frac{3}{2}}}$. The continuity dependence with respect to $\mathbf{Q}$ is classical and follows from the fixed point theorem with a parameter. This achieves the proof of the lemma.

Therefore equation (6.43) admits a solution in the form

$$
X=X_{+}(\mathbf{Q})=\sqrt{\frac{a}{\tilde{c}} \varepsilon^{2}+\frac{\tilde{b}}{\tilde{c}} \mathbf{Q}^{2}}+y(\mathbf{Q}) .
$$

Reproducing the same analysis we can prove that the equation (6.43) admits another solution of the form

$$
X_{-}(\mathbf{Q})=-\sqrt{\frac{a}{\tilde{c}} \varepsilon^{2}+\frac{\tilde{b}}{\tilde{c}} \mathbf{Q}^{2}}+\widehat{y}(\mathbf{Q}), \quad \widehat{y}(\mathbf{Q}) \in \bar{B}_{\eta \varepsilon^{\frac{3}{2}}} .
$$

It remains only to check that the curves $\mathbf{Q} \in \bar{B}_{\eta \varepsilon^{\frac{5}{6}}} \mapsto X_{ \pm}$are disjoint graphs. For this we write, by the triangular inequality,

$$
\begin{aligned}
\left|X_{+}(\mathbf{Q})-X_{-}(\mathbf{Q})\right| & \geq 2 \sqrt{\frac{a}{\tilde{c}} \varepsilon^{2}+\frac{\tilde{b}}{\tilde{c}} \mathbf{Q}^{2}}-(|y(\mathbf{Q})|+|\widehat{y}(\mathbf{Q})|) \\
& \geq C\left(|\varepsilon|-2 \eta|\varepsilon|^{\frac{3}{2}}\right) \\
& >C \varepsilon
\end{aligned}
$$

if $\varepsilon$ is small enough. Coming back to the initial unknowns, we find two disjoint curves of solutions to equation (6.42). The proof of Theorem 6.5 is now complete.

\section{Conclusions}

In this paper we have described both numerically and analytically the bifurcation structure of twofold and three-fold symmetric singly-connected vortex patch equilibria for the Quasi-Geostrophic Shallow-Water equations. The numerical results reveal that the branch of solutions for two-fold symmetric equilibria, consisting of a main branch of Kirchhoff elliptical vortices and secondary branches bifurcating at the Love instability points for the Euler equations, likely separates into an infinite set of disjoint branches for any finite value of the Rossby deformation length $\varepsilon^{-1}$. This is confirmed for $\varepsilon \ll 1$ by mathematical analysis for the first separation near the Love instability point for elliptical azimuthal wavenumber $m=4$.

The numerical results for the three-fold symmetric equilibria also reveal a separated branch, in this case existing for all $\varepsilon$. The mathematical analysis confirms that solutions exist for small $\varepsilon$ near the Euler limit $(\varepsilon=0)$; these solutions are near circular in form. The separated solutions are 
beyond the scope of this analysis. In a future work, we will report on the stability and nonlinear evolution of these solutions.

Acknowledgements. DGD received support for this research from the UK Engineering and Physical Sciences Research Council (grant number EP/H001794/1). TH is partially supported by the the ANR project Dyficolti ANR-13-BS01-0003- 01.

\section{REFERENCES}

[1] J. Burbea. Motions of vortex patches. Lett. Math. Phys. 6, no. 1, 1-16 (1982).

[2] A. Castro, D. Córdoba, J. Gomez-Serrano. Existence and regularity of rotating global solutions for the generalized surface quasi-geostrophic equations. Duke Math. J. 165, no. 5, 935-984 (2016).

[3] A. Castro, D. Córdoba, J. Gomez-Serrano. Uniformly rotating analytic global patch solutions for active scalars. Ann. PDE 2, no. 1, Art. 1, 34 pp (2016).

[4] C. Cerretelli, C. H. K. Williamson. A new family of uniform vortices related to vortex configurations before merging. J. Fluid Mech. 493, 219-229 (2003).

[5] M. G. Crandall, P. H. Rabinowitz. Bifurcation from simple eigenvalues J. of Func. Analysis 8, 321-340 (1971).

[6] G. S. Deem, N. J. Zabusky. Vortex waves: Stationary "V-states", Interactions, Recurrence, and Breaking. Phys. Rev. Lett. 40, no. 13, 859-862 (1978).

[7] D. G. Dritschel. The nonlinear evolution of rotating configurations of uniform vorticity. J. Fluid Mech. 172, 157-172 (1986).

[8] D. G. Dritschel. Contour surgery: a topological reconnection scheme for extended integrations using contour dynamics. J. Comput. Phys. 77, 240-266 (1988).

[9] D. G. Dritschel. Contour dynamics and contour surgery: numerical algorithms for extended, high-resolution modelling of vortex dynamics in two-dimensional, inviscid, incompressible flows. Computer Phys. Rep. 10, 77146 (1989).

[10] D. G. Dritschel. A general theory for two-dimensional vortex interactions. J. Fluid Mech. 293, 269-303 (1995).

[11] M. Golubitsky, D. Schaeffer, A theory for imperfect bifurcation via singularity theory. Comm. Pure Appl. Math. 32 (1979), no. 1, 21-98.

[12] Z. Hassainia, T. Hmidi. On the V-states for the generalized quasi-geostrophic equations. Comm. Math. Phys. 337, no. 1, 321-377 (2015).

[13] Z. Hassainia, N. Masmoudi, M. H. Wheeler, Global bifurcation of rotating vortex patches, arXiv:1712.03085

[14] T. Hmidi, J. Mateu, J. Verdera. Boundary Regularity of Rotating Vortex Patches. Arch. Ration. Mech. Anal. 209, no. 1, 171-208 (2013).

[15] T. Hmidi, J. Mateu. Bifurcation of rotating patches from Kirchhoff vortices. Discrete Contin. Dyn. Syst. 36 (2016) no. 10, 5401-5422.

[16] J. R. Kamm. Shape and stability of two-dimensional uniform vorticity regions. PhD thesis, California Institute of Technology, 1987.

[17] G. R. Kirchhoff. Vorlesungenb̈er mathematische Physik. Mechanik. Teubner, Leipzig (1876).

[18] P. Liu, J. Shi,Y. Wang, Imperfect transcritical and pitchfork bifurcations. J. Funct. Anal. 251 (2007), no. 2, 573-600.

[19] A. E. H. Love. On the stability of certain vortex motions. Proc. Lond. Math. Soc. 35, 18 (1893).

[20] P. Luzzatto-Fegiz, C. H. K. Williamson. Stability of elliptical vortices from "Imperfect-Velocity-Impulse" diagrams. Theor. Comput. Fluid Dyn. 24, no. 1-4, 181-188 (2010).

[21] P. Luzzatto-Fegiz, C. H. K. Williamson. An efficient and general numerical method to compute steady uniform vortices. J. Comput. Phys. 230, 6495-6511 (2011).

[22] E. A. Overman II. Steady-state solutions of the Euler Equations in two dimensions II. Local analysis of limiting $V$-states. SIAM J. Appl. Math. 46, no. 5, 765-800 (1986).

[23] H. Płotka, D. G. Dritschel. Quasi-geostrophic shallow-water vortex-patch equilibria and their stability. Geophys. Astrophys. Fluid Dyn. 106, no. 6, 574-595 (2012).

[24] L. M. Polvani. Geostrophic vortex dynamics. PhD thesis, MIT/WHOI WHOI-88-48 (1988).

[25] L. M. Polvani, N. J. Zabusky, G. R. Flierl. Two-layer geostrophic vortex dynamics. Part 1. Upper-layer V-states and merger. J. Fluid Mech. 205, 215-242 (1989).

[26] J. Segura. Bounds for ratios of modified Bessel functions and associated Turán-type inequalities. J. Math. Anal. Appl. 374, pp. 516-528 (2011).

[27] J. Shi, Persistence and bifurcation of degenerate solutions. J. Funct. Anal. 169 (1999), no. 2, $494-531$.

[28] G. K. Vallis. Atmospheric and Oceanic Fluid Dynamics. Cambridge University Press (2008). 
[29] G. N. Watson. A Treatise on the Theory of Bessel Functions. Cambrige University Press (1944).

Mathematical Institute, University of St Andrews, St Andrews KY16 9SS, UK

E-mail address: david.dritschelest-andrews.ac.uk

Univ Rennes, CNRS, IRMAR - UMR 6625, F-35000 Rennes, France

E-mail address: thmidi@univ-rennes1.fr

Univ Rennes, CNRS, IRMAR - UMR 6625, F-35000 Rennes, FranCE

E-mail address: Coralie.Renault@ens-rennes.fr 